\title{
TOPOLOGICAL PROPERTIES OF REAL ALGEBRAIC VARIETIES: DU COTE DE CHEZ ROKHLIN
}

\author{
A. Degtyarev and V. Kharlamov \\ To Vladimir Abramovich Rokhlin
}

\section{Contes de la Vieille Grand' Mère}

Иные воспоминания наполовину стёрлись в её памяти, другие не сотрутся никогда. ${ }^{1}$

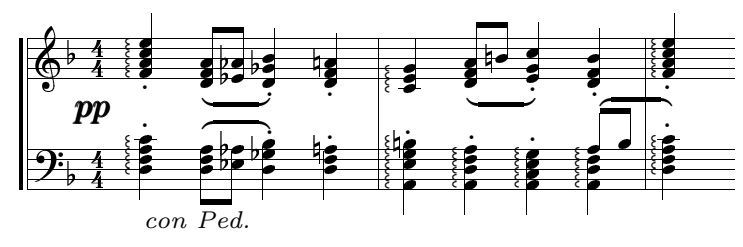

С. ПРОКОФЬЕВ. Соч. 31 (1918)

Abstract. The survey gives an overview of the achievements in topology of real algebraic varieties in the direction initiated in the early 70th by V. I. Arnold and V. A. Rokhlin. We make an attempt to systematize the principal results in the subject. After an exposition of general tools and results, special attention is paid to surfaces and curves on surfaces.

\section{Contents}

1. Introduction 2

2. General tools and results 7

3. Surfaces 19

4. Curves on Surfaces

Appendix A. Topology of involutions

Appendix B. Integral lattices and quadratic forms

1991 Mathematics Subject Classification. 14P25, 14J99, and 57S25.

Key words and phrases. Real algebraic variety, real algebraic surface, real algebraic curve, Hilbert's 16th problem.

The title imitates that of M. Proust's novel, usually translated as Swan's way (cf. also similar play on words in L. Guillou, A. Marin, A la recherche de la topologie perdue).

${ }^{1}$ Some of her memories are half gone; others will stay forever. S. Prokofiev, Op. 31 (1918) 


\section{INTRODUCTION}

The break-through. To great extent, the interest shown by Vladimir Abramovich Rokhlin to topology of real algebraic varieties was motivated by the results obtained in the late 60 th by D. Gudkov [GUt] and the subsequent paper by V. Arnol'd [A3], which made a considerable contribution to the solution of Hilbert's 16th problem. Gudkov disproved one of Hilbert's conjectures on the arrangement of ovals (i.e., two-sided components) of plane $M$-sextics (i.e., sextics with the maximal number of ovals). He corrected the conjecture, proved it for degree 6, and suggested [G1], as a new conjecture, an extension of his result to $M$-curves of any even degree. (Recall that an $M$-curve of genus $g$ is a curve with the maximal number of connected components of the real part; due to Harnack's bound the maximal number is $M=g+1$.)

To state Gudkov's conjecture, recall that an oval of a plane curve is called even (odd) if it lies inside an even (respectively, odd) number of other ovals. The number of even (odd) ovals is denoted by $p$ (respectively, $n$ ). In this notation Gudkov's conjecture claims that $p-n=k^{2} \bmod 8$ for any $M$-curve of degree $2 k$.

V. Arnol'd in his remarkable paper [A3] related the study of real plane curves to topology of 4-manifolds and arithmetics of integral quadratic forms and, besides other results, proved a weaker version of Gudkov's conjecture $\left(p-n=k^{2} \bmod 4\right)$. After that the events were developing swiftly. First, Rokhlin suggested a proof of Gudkov's conjecture based on his formula relating the signature of a 4-manifold with the Arf-invariant of a characteristic surface (see Appendix C). Then, after Kharlamov's [Kh1] generalization of Arnol'd's results to surfaces, Rokhlin [R4] found another proof, not using any specific tools of 4-dimensional topology, and extended the statement to varieties of any dimension. The revolutionary break-through originated by Arnol'd was over and a period of systematic study started. (More details and a brief account of the further history of the subject can be found in [G2], [Wi], and $[\mathrm{AO}]$.

Rokhlin's heritage. Rokhlin published six papers on topology of real algebraic varieties (see [R1]-[R6]). This number is not very large, but each of these papers originated a whole new direction in the subject. (The only exception is probably the short note [R5], which extended the range of applications of some previous results from complete intersections to arbitrary real algebraic varieties.) In this survey we discuss, in more or less details, all papers except [R6], where nonalgebraic coverings are used to study algebraic curves; for other examples of this approach see [Fi3].

In Rokhlin's first paper [R1] there is a mistake in the proof of Gudkov's conjecture. However, the approach of the paper, namely, using characteristic surfaces in a 4 -manifold to evaluate its signature $\bmod 16$, became a powerful method in the study of real algebraic curves. It was used by A. Marin, who, together with L. Guillou (see $[\mathrm{GM}]$ ), extended Rokhlin's signature formula to nonorientable characteristic surfaces, and by means of it corrected the mistake. (The Rokhlin-Guillou-Marin formula and related quadratic form are discussed in Appendix C.)

Another fundamental result, difficult to overestimate, is Rokhlin's formula of complex orientations. As soon as observed, the notion of complex orientation of a dividing real curve (see below), as well as Rokhlin's formula and its proof, seem incredibly transparent. The formula settles, for example, two of D. Hilbert's conjectures on 11 ovals of plane sextics, which the latter tried to prove in a very 
sophisticated way and included into his famous problem list (as 16-th problem). Nowadays Rokhlin's formula is a major tool in the study of the topology of real curves on surfaces. It is one of the few phenomena related to curves that still do not have a satisfactory generalization to varieties of higher dimension. (In fact, even for curves on surfaces some basic questions have not been clarified completely yet, cf. 4.2.)

It is worth mentioning that it was F. Klein who first studied dividing curves. He discovered some of their remarkable properties and pointed out a way to improve and generalize Harnack's bound on the number of connected components for such curves. However, he did not notice the complex orientations.

Rokhlin's formula of complex orientation and Hilbert's 16th problem. An irreducible over $\mathbb{R}$ nonsingular real curve $A$ (i.e., either an irreducible nonsingular complex curve with an antiholomorphic involution conj: $A \rightarrow A$ or a nonsingular complex curve consisting of two irreducible components transposed by an antiholomorphic involution) is called dividing or of type $\mathrm{I}$, if its real part $\mathbb{R} A=$ Fix conj divides $A$ into two halves: two connected 2-manifolds $A_{+}$and $A_{-}$having $\mathbb{R} A$ as their common boundary. (Note that if $A$ is irreducible over $\mathbb{R}$ but reducible over $\mathbb{C}$, it is dividing.) The complex conjugation conj: $A \rightarrow A$ interchanges $A_{ \pm}$and the complex orientation of $A_{ \pm}$induces two opposite orientations on $\mathbb{R} A$, called its complex orientations. If the curve is reducible or singular, it is called dividing if so is the normalization of each real component. Clearly, $A$ is dividing if and only if the quotient $A /$ conj is orientable, and if this is the case, the complex orientations are induced by orientations of $A$ /conj. The principal example is provided by $M$-curves, which are all dividing.

Let $A$ be a nonsingular dividing plane curve. Two ovals are said to form an injective pair if they bound an annulus in $\mathbb{R P}^{2}$. An injective pair is called positive if the complex orientations of the ovals are induced from an orientation of the annulus, and negative otherwise. Denote by $\Pi^{+}$and $\Pi^{-}$the numbers of, respectively, positive and negative injective pairs, and let $\Pi=\Pi^{+}+\Pi^{-}$.

1.1. Rokhlin's formula. For a nonsingular dividing plane curve of even degree $2 k$ with $l$ real components one has $2\left(\Pi^{+}-\Pi^{-}\right)=l-k^{2}$.

Rokhlin's formula has numerous applications. For example, the Arnol'd and Slepian congruences are its straightforward consequences. Given a dividing curve of degree $2 k$, the Arnol'd congruence states that $p-n=k^{2} \bmod 4$, and the Slepian congruence states that $p-n=k^{2} \bmod 8$ provided that each odd oval contains immediately inside it an odd number of even ovals (so that it bounds from outside a component of the complement of the curve with even Euler characteristic). Another consequence of Rokhlin's formula is the fact that for any dividing curve of degree $2 k$ one has $\Pi \geqslant\left|l-k^{2}\right|$ and $l \geqslant k$. The latter, together with the Klein congruence $l=k \bmod 2$, which also follows from Rokhlin's formula, is the complete set of restrictions on the number of components of a nonsingular dividing plane curve of degree $2 k$; for curves of degree $2 k-1$ the corresponding version of Rokhlin's formula (Rokhlin-Mishachev formula, see, e.g., [R3]) implies $l \geqslant k$ and a similar statement holds. ${ }^{2}$

\footnotetext{
${ }^{2}$ As we learned from A. Gabard, the problem on the number of components of a dividing curve of a given degree was first posed by F. Klein.
} 
Given a dividing curve $A$, its real scheme (i.e., the topological type of $\left(\mathbb{R} \mathbb{P}^{2}, \mathbb{R} A\right)$ ) and the complex orientation determine $A \cup \mathbb{R} \mathbb{P}^{2}$ up to homeomorphism. (If the curve is not dividing, $A \cup \mathbb{R} \mathbb{P}^{2}$ is determined by the real scheme of $A$.) One of the related problems, suggested by Rokhlin, is the study of complex orientations appearing in a given degree; it occupies an intermediate position between the stronger question on the equivariant topology of the pair $\left(\mathbb{P}^{2}, A\right)$ and the weaker one on $\left(\mathbb{R} \mathbb{P}^{2}, \mathbb{R} A\right)$ (the original setting of Hilbert's 16th problem). Two other related problems are worth mentioning here: the study of the fundamental group $\pi_{1}\left(\mathbb{P}^{2} \backslash\left(\mathbb{R} \mathbb{P}^{2} \cup \mathbb{R} A\right)\right)$ and of the topology of the subspace $\left(\mathbb{R P}^{2} \cup A\right) \subset \mathbb{P}^{2} /$ conj $=S^{2}$.

Rokhlin's school. Rokhlin's influence on topology of real algebraic varieties extends far beyond his own results. He directed into the subject a number of his students (Cheponkus, Fiedler, Finashin, Kharlamov, Mischachev, Slepian, Viro, Zvonilov) and generously shared his ideas and broad knowledge. Unfortunately, Vladimir Abramovich could not realize all his plans: his untimely death interrupted his work on quite a number of projects.

As early as in the middle seventies Rokhlin had an intention to write two detailed surveys of the results achieved by that time: one on real plane curves, and another one on higher dimensional varieties. At the very beginning the project was joined by V. Kharlamov and later, by O. Viro. Detailed plans were elaborated, Rokhlin wrote summaries of the first chapters of the book on plane curves, and Kharlamov, summaries of the first chapters of the other book. Rokhlin was planning to deliver graduate courses in Leningrad State University; unfortunately, he was forced to retire, and the course on plane curves was given first by Viro (in a shorten version) and then by Viro and Kharlamov. Kharlamov and Viro continued the work on the first project after Rokhlin had passed away. The work was interrupted several times, due to various political and geographical reasons. However, a draft of the book has been written and a considerable part of it has been polished. A preliminary version of the book has been used by a number of graduate students as an introduction to the subject. Pitifully, it seems that the book will never appear (partially due to the fact that the subject is developing faster than the text is being written). The introductory section of Viro's survey [V1] can give one an idea about the first chapters of the book. (The survey, which is mainly devoted to other, construction aspects of the subject, gives a broader coverage of the principal notions and results, while the book treats the subtle details that are only appropriate for a graduate text.) The present survey reflects the plan of the second project; in its preliminary form it was designed as an appendix to the book on curves (and, naturally, much inspired by it; many results in 4.6 and 4.7 are straightforward generalizations of the corresponding results for curves treated in the book).

What is and what is not contained in the survey. We do not intend to give a complete overview of all known results on topology of real algebraic varieties: the subject is so developed and divers that it seems impossible to cover it within a reasonable volume. Instead, we tried to select the results more closely related to the phenomena discovered and studied by Rokhlin and his followers (certainly, even this choice is not exhaustive and is mainly due to our personal taste) and made an attempt to illustrate how the approaches and techniques developed work. Most proofs are either omitted or just sketched. The citation list is also incomplete; in many cases we refer to papers and textbooks where the concepts and proofs are presented in a suitable way rather than to the original works. 
Historically a great deal of attention was paid to the case of plane curves; we address the reader to the excellent surveys [G2], [Wi], [V1] and concentrate on the results in higher dimensions. However, at the end we return to surfaces and curves on surfaces and discuss a few later developments, especially those related to complex orientations. (Note that the topological properties of abstract, not embedded, real curves are simple and have been understood completely since F. Klein, see, e.g., [R3], [Na].) A separate topic are real 3-folds. For a long time they stayed outside the main scope of the subject; recently, due to J. Kollár, the situation started changing, and now the 3 -folds should deserve a separate paper.

It turns out that most known results for plane curves are derived from appropriate results for certain auxiliary surfaces; moreover, in many cases the more general higher dimensional setting is, in fact, more suitable as it gives proper understanding of the relation between the topology of the real part of a variety and its complexification. However, there are few exceptions, which are still not extended to higher dimensions; the most remarkable of them is Rokhlin's formula of complex orientations, mentioned above, and, among newer developments, results by S. Orevkov, based on a systematic study of pencils of lines. (Orevkov's results, except his formula of complex orientations 4.5.1, mainly deal with curves of low degrees; their general meaning and relation to other approaches has not been completely revealed yet, even for plane curves.)

Topology of real algebraic varieties is developing in two directions: prohibitions and constructions. In this survey we completely ignore the latter (see, e.g., [V1], [IV1], [Ri] for an overview of corresponding methods and results) and concentrate on the prohibition type results, i.e., the restrictions on the topology of the real point set of a real algebraic variety imposed by the topology of its complex point set. The latter is usually assumed known; a typical example is considering varieties within a fixed complex deformation family.

Some other important topics, currently developing but ignored in this survey, are: special polynomials, fewnomials, complexity, singularities and singular varieties, approximations, metric properties, Ax principle, toric varieties, algebraic cycles, moduli spaces, minimal models, relations to symplectic geometry.

Although our main subject are real algebraic varieties, many results extend to much wider categories. In particular, instead of algebraic varieties defined over $\mathbb{R}$ we often consider closed complex manifolds supplied with an anti-holomorphic involution. In many cases the complex structure does not need to be integrable. (The most intriguing exception is the generalized Comessatti-Petrovsky-Oleinik inequality 2.4.1, which has a topological proof for surfaces, while the known proofs for higher dimensional varieties use the integrability of the complex structure and even the existence of a Kähler form.) Moreover, many prohibition results are topological in their nature and, thus, hold for arbitrary smooth manifolds with involution; sometimes one needs to assume, in addition, that locally the fixed point set of the involution behaves as the real point set of a real algebraic variety, i.e., its normal and cotangent bundles are isomorphic. These phenomena are not extremely surprising, considering the modern reconciliation of differential and algebraic geometries. One can also speculate on the unity of complex and real algebraic geometries, based, e.g., on the correspondence $X \mapsto X \times \bar{X}$ ( $\bar{X}$ standing for the complex conjugate variety) or $X \mapsto X \sqcup \bar{X}$; in the latter case there is a bijection between the real structures $f: X \rightarrow \bar{X}$ and involutive automorphisms of $X \sqcup \bar{X}$ of the form $f \sqcup \bar{f}$. 
Notation. A real structure on a complex analytic manifold $X$ is an anti-holomorphic involution. (In the case of algebraic varieties defined over $\mathbb{R}$ it is the Galois involution.) Usually, we denote it by conj: $X \rightarrow X$. The fixed point set of the real structure is called the real part of the variety and denoted by $\mathbb{R} X$. The quotient space $X /$ conj is usually denoted by $X^{\prime}$.

As it has been mentioned above, many results are topological in their nature and, thus, hold for wider classes of varieties. Although it is difficult to incorporate all the necessary hypotheses in a single statement, in most cases the following definition is quite suitable: a flexible real variety is a closed smooth manifold $X$ supplied with a smooth involution conj: $X \rightarrow X$ and a (not necessarily integrable) quasicomplex structure $J$ in a neighborhood of the real part Fix conj, so that conj is anti-holomorphic in respect to $J$. (When the Bezout theorem is concerned, it may also be useful to consider a symplectic structure compatible with both conj and $J$.)

A closed complex (not necessarily irreducible or reduced) submanifold $A \subset X$ is called real if it is conj-invariant. To avoid the confusion between conj-invariant complex submanifolds and real submanifolds in the ordinary sense, we sometimes call the former real smooth cycles (or real smooth divisors, if the complex codimension is 1). A curve in a complex manifold $X$ is a reduced effective cycle (divisor, if $X$ is a surface).

Many notions in algebraic geometry have different meaning over $\mathbb{C}$ and over $\mathbb{R}$; in order to designate the real version we use the prefix $\mathbb{R}$. Thus, a submanifold $A$ is $\mathbb{R}$-irreducible if either it is irreducible over $\mathbb{C}$ or it has two components permuted by conj; an $\mathbb{R}$-component of $A$ is either a conj-invariant component or a pair of components permuted by conj. (Realizing the awkwardness of this terminology, we adopt it as we could not find anything better. Designating these notions as 'real' might cause confusion with their topological counterparts, while 'over $\mathbb{R}$ ' is not always applicable, grammatically or, even worse, semantically, as in general our manifolds are not algebraic varieties defined over $\mathbb{R}$.)

Given a linear system $|D|$ (see 2.1) on a complex manifold $X$ with real structure, we denote by $\mathbb{R}|D|$ its real part, which consists of the real divisors linearly equivalent to $D$, and by $\Delta_{|D|}$ and $\mathbb{R} \Delta_{|D|}$, the discriminant of $|D|$ and its real part, respectively. In the particular case $X=\mathbb{P}^{n}$ we use the more common notation $\mathcal{C}_{q}, \Delta_{q}$ for the space of hypersurfaces of degree $q$ and its discriminant, respectively, and $\mathbb{R} \mathcal{C}_{q}, \mathbb{R} \Delta_{q}$ for their real parts.

We use the standard bracket notation to encode finite partially ordered sets. Typically this notation is applied to the set of ovals of a real curve on a real surface. The particular partial order used is to be specified explicitly for each class of curves; e.g., for two ovals $C_{1}, C_{2}$ of a plane curve one has $C_{1} \succ C_{2}$ if $C_{2}$ belongs to the disk bounded by $C_{1}$.

Unless stated explicitly, all the homology and cohomology groups have coefficients $\mathbb{Z}_{2}$. We use $b_{i}(\cdot)$ and $\beta_{i}(\cdot)$ for the Betti numbers over $\mathbb{Q}$ and over $\mathbb{Z}_{2}$, respectively, and $b_{*}(\cdot)$ and $\beta_{*}(\cdot)$, for the corresponding total Betti numbers. Given a topological space $X$ with involution $c$, we denote by $\left({ }^{r} E_{*}(X),{ }^{r} d_{*}\right)$ (or just $\left({ }^{r} E_{*},{ }^{r} d_{*}\right)$ ) Kalinin's spectral sequence of $X$, by bv ${ }_{*}$, the Viro homomorphisms, and by $\mathcal{F}^{*}(X)=$ $\mathcal{F}^{*}$, Kalinin's filtration on $H_{*}($ Fix $c)$ (see A.2).

If $X$ is a manifold, $w_{i}=w_{i}(X)$ stand for its Stiefel-Whitney classes, $u_{i}=$ $u_{i}(X)$, for its $\mathrm{Wu}$ classes, and, if $X$ is complex, $c_{i}=c_{i}(X)$, for its Chern classes. If $X$ is closed, we denote by $\mathrm{D}=\mathrm{D}_{X}: H^{*}(X) \rightarrow H_{*}(X)$ the Poincaré duality isomorphism and often use the same notation $w_{i}, u_{i}, c_{i}$ for the Poincaré dual 
homology characteristic classes.

Acknowledgements. We are grateful to our numerous colleagues with whom we worked on various topics in the subject and who shared with us their knowledge. The list of persons who helped us to clarify certain details or communicated to us their unpublished results during our work on this survey includes, but is not limited to, B. Chevallier, S. Finashin, I. Itenberg, A. Marin, S. Orevkov, O. Viro, J.-Y. Welschinger. Our particular gratitude is to A. M. Vershik, who encouraged us to write this survey.

\section{General tools AND Results}

2.1. Divisors and linear equivalence. Recall that a divisor on a nonsingular compact complex manifold $X$ is a formal finite integral linear combination $A=\sum m_{i} D_{i}$, where $m_{i} \in \mathbb{Z}$ and $D_{i}$ are irreducible (possibly singular) compact codimension 1 subvarieties of $X$. If all $m_{i} \geqslant 0, A$ is called effective. Divisors form a group in respect to the formal addition of linear combinations. Two divisors $D_{1}$, $D_{2}$ are called linearly equivalent, $D_{1} \sim D_{2}$, if their difference is a principal divisor, i.e., there is a meromorphic function on $X$ whose zeros and poles are the components of $D-D^{\prime}$, considered with their multiplicities. All effective divisors linearly equivalent to a given divisor $D$ form a projective space; it is called a linear system and denoted by $|D|$. Clearly, linearly equivalent divisors realize the same class in $H^{2}(X)$; if $X$ is simply connected (or, more generally, $H^{1}(X)=0$ ), this condition is also sufficient (see, e.g., [H2] or [Ha]).

A divisor is called very ample if it can be realized as a hyperplane section under an appropriate embedding of $X$ into a projective space. A divisor $D$ is called ample if some positive multiple $m D$ is very ample. (Some useful criteria of ampleness can be found in [Ha].)

If $A$ is a real divisor on a real variety $\left(X\right.$, conj), the class of $A$ in $H^{2}(X ; \mathbb{Z})$ is conj*-skew-invariant. If $H^{1}(X)=0$, every divisor whose class is conj ${ }^{*}$-skewinvariant is linearly equivalent to a real one. Real divisors equivalent over $\mathbb{C}$ are equivalent over $\mathbb{R}$, provided that $\mathbb{R} X \neq \varnothing$.

2.1.1. Lefschetz theorem on hyperplane section (see, e.g., [MS]). If $A$ is an ample divisor on a compact complex $n$-dimensional manifold $X$, then $X \backslash A$ has homotopy type of $n$-dimensional $C W$-complex. In particular, for any abelian group $G$ and $r<n$ one has $H_{r}(X, A ; G)=H^{r}(X, A ; G)=0$.

2.2. Double coverings. Double coverings play a special rôle in real algebraic geometry: the position of a subvariety $A \subset X$ is reflected, to great extent, in the topological properties of the double covering of $X$ ramified in $A$ (according to V. Arnol'd's principle, the notion of double covering is the complexification of that of manifold with boundary; Arnol'd claims that it is due to this observation that he found a proof of relaxed Gudkov's congruence and the other remarkable results of [A1]). Thus, once an absolute result is obtained, it may be applied to branched double coverings and thus produce some relative prohibitions on the position of a subvariety.

A divisor $A \subset X$ is called even if the fundamental class $[A]$ vanishes in $H_{2 n-2}(X)$, where $n=\operatorname{dim} X$. In this case there exists a double covering $Y$ of $X$ branched over $A$, which is also a complex variety, nonsingular if so is $A$. Clearly, an isomorphism class of the covering is determined by a class $\omega \in H^{1}(X \backslash A)$ whose image 
under $H^{1}(X \backslash A) \rightarrow H_{2 n-1}(X, A) \rightarrow H_{2 n-2}(A)$ is $[A]$. Alternatively, the homology vanishing condition is equivalent to $A \sim 2 E$ for some divisor $E$ on $X$, and the isomorphism classes of double coverings are in a canonical one-to-one correspondence with the classes $E \in \operatorname{Pic} X$ such that $2 E \sim A$, see [H1]. To indicate a particular choice of the covering we use the notation $Y(E)$.

The following is an immediate consequence of 2.1.1:

2.2.1. Lefschetz theorem for double covering. Let $A$ be an even ample divisor on a compact complex manifold $X$ and $Y$ a double covering of $X$ branched over $A$. Then for any abelian group $G$ one has

$$
H_{r}(Y, A ; G)=H^{r}(Y, A ; G)=0 \quad \text { for } r<n=\operatorname{dim} X .
$$

2.2.2. Corollary of 2.1 .1 and 2.2.1. Let $A \subset X$ and $Y$ be as in 2.2 .1 and $p: Y \rightarrow X$ the covering projection. Then for any abelian group $G$ the induced map $p_{*}: H_{r}(Y ; G) \rightarrow H_{r}(X ; G)$ is an isomorphism for $r<n$ and an epimorphism for $r=n$; the induced map $p^{*}: H^{r}(X ; G) \rightarrow H^{r}(Y ; G)$ is an isomorphism for $r<n$ and a monomorphism for $r=n$. Furthermore, if $G$ is a field, char $G \neq 2$, then $p_{*}$ induces an isomorphism between $H_{*}(X ; G)$ and the $(+1)$-eigenspace $H_{*}^{+}(Y ; G)$ of the deck translation (respectively, $p^{*}$ induces an isomorphism between $H^{*}(X ; G)$ and $\left.H_{+}^{*}(Y ; G)\right)$.

Note that 2.2.1 and 2.2.2 still hold if $A$ and, hence, $Y$ are singular. If $A$ is smooth but not even, one may try to find another smooth divisor $H$ on $X$, intersecting $A$ transversally, so that $[H]+[A]$ vanishes in $H_{2 n-2}(X)$. Then there is a double covering $Y_{H}$ of $X$ branched along $H \cup A$, and one can study its blow-up $\widetilde{Y}_{H}$ at $S=$ $H \cap A$. (A typical example is when $X \subset \mathbb{P}^{N}$ and $A$ is cut on $X$ by a hypersurface of odd degree: taking for $H$ a generic hyperplane section one obtains information about an affine part of $(X, A)$.) Equivalently, one can first blow up $S$ at $X$ to obtain a manifold $\widetilde{X}$; then $\widetilde{Y}$ is the double covering of $\widetilde{X}$ branched along the proper transform of $A+H$. If $H$ is ample, so is $a H+A$ for $a \gg 0$, and since $H_{r}(\widetilde{X})=H_{r}(X) \oplus H_{r-2}(S)$, Corollary 2.2.2 takes the following form:

2.2.3. Proposition. Let $A \subset X$ be a smooth divisor, $H \subset X$ an ample divisor transversal to $A$, and $\widetilde{Y}_{H}$ as above. Then for any field $F$, char $F \neq 2$, and any $r \in \mathbb{Z}$ there are canonical isomorphisms $H_{r}^{+}\left(\widetilde{Y}_{H} ; F\right)=H_{r}(X ; F) \oplus H_{r-2}(H \cap A ; F)$ and $H_{+}^{r}\left(\widetilde{Y}_{H} ; F\right)=H^{r}(X ; F) \oplus H^{r-2}(H \cap A ; F)$. Furthermore, $H_{r}^{-}\left(\widetilde{Y}_{H} ; F\right)=$ $H_{-}^{r}\left(\widetilde{Y}_{H} ; F\right)=0$ for $r \neq n=\operatorname{dim} X$. (Here $H_{*}^{ \pm}$and $H_{ \pm}^{*}$ are the $( \pm 1)$-eigenspaces of the deck translation involution.)

2.2.4. Assume now that $X$ is supplied with a real structure and $A$ is an even real divisor. Assume, further, that $E$ with $2 E \sim A$ is also chosen real and let $Y=Y(E)$. (Such a choice of $E$ exists, e.g., if $H^{1}(X)=0$; in general the existence depends on whether the class of $A$ in $H^{2}(X ; \mathbb{Z})$ is the double of a skew-invariant class.) If $\mathbb{R} X \neq \varnothing$, the involution conj on $X$ lifts to two involutions $T_{ \pm}: Y \rightarrow Y$, which are antiholomorphic, commute with each other and with the deck translation $\tau: Y \rightarrow Y$, and satisfy $\tau=T_{+} \circ T_{-}$(see A.2.5). The corresponding real parts of $Y$ are denoted by $\mathbb{R} Y_{ \pm}$, and their projections to $\mathbb{R} X$, by $\mathbb{R} X_{ \pm}$. Obviously, $\mathbb{R} X_{+} \cup$ $\mathbb{R} X_{-}=\mathbb{R} X$ and $\mathbb{R} X_{+} \cap \mathbb{R} X_{-}=\mathbb{R} A$. The characteristic classes $\omega_{ \pm} \in H^{1}\left(\mathbb{R} X_{ \pm}\right)$ are Poincaré dual to the corresponding restrictions $\operatorname{rel}[\mathbb{R} E] \in H_{n-1}\left(\mathbb{R} X_{ \pm}, \mathbb{R} A\right)$. 
2.3. Smith inequalities. This group of results, obtained from the Smith inequality and its relative version (see A.1.3(2) and (A.1.9), respectively), generalizes the Harnack inequality for curves.

2.3.1. Theorem. For a manifold $X$ with real structure one has $\beta_{*}(\mathbb{R} X) \leqslant \beta_{*}(X)$ and $\beta(\mathbb{R} X)=\beta(X) \bmod 2$. If, furthermore, $A \subset X$ is a real submanifold, then $\beta_{*}(\mathbb{R} X, \mathbb{R} A) \leqslant \beta_{*}(X, A)$ and $\beta_{*}(\mathbb{R} X, \mathbb{R} A)=\beta(X, A) \bmod 2$.

In 2.3.1 one can easily recognize the familiar relations between the number of real roots of a real polynomial and its degree, if $\operatorname{dim} X=0$, and the classical Harnack inequality, which states that the number of real components of $\mathbb{R} X$ does not exceed genus $(X)+1$, if $\operatorname{dim} X=1$. The bound given by 2.3.1 is sharp for hypersurfaces of any degree in projective spaces of any dimension (see [V2] and the forthcoming [IV2]); for a hypersurface $X \subset \mathbb{P}^{q}$ of degree $m$ the bound takes the form $\beta_{*}(X) \leqslant \frac{1}{m}\left((m-1)^{q+1}+(-1)^{q}\right)+q-(-1)^{q}$, see [Th3].

2.3.2. Complementary inequality for even pairs. Let $A$ be an even ample real submanifold on a manifold $X$ with real structure, $\operatorname{dim} X=n$. Let, further, $E$ be a real divisor on $X$ with $2 E \sim A$ and $\omega_{ \pm} \in H^{1}\left(\mathbb{R} X_{ \pm}\right)$the class Poincaré dual to $\operatorname{rel}[\mathbb{R} E] \in H_{n-1}\left(\mathbb{R} X_{ \pm}, \mathbb{R} A\right)$, where $\mathbb{R} X_{ \pm}$are the two halves of $\mathbb{R} X \backslash \mathbb{R} A$ (see. 2.2.4). Then

$$
2 \beta_{*}\left(\mathbb{R} X_{ \pm}\right)-2\left[\operatorname{dim} \operatorname{Ker} \partial-\operatorname{dim} \operatorname{Ker}\left(\omega_{ \pm} \oplus \partial\right)\right] \leqslant \beta_{*}(X)+(-1)^{n}[\chi(X)-\chi(A)],
$$

where $\omega_{ \pm} \oplus \partial: H_{*}\left(\mathbb{R} X_{ \pm}, \mathbb{R} A\right) \rightarrow H_{*-1}\left(\mathbb{R} X_{ \pm}, \mathbb{R} A\right) \oplus H_{*-1}(\mathbb{R} A)$ is the boundary homomorphism in the Smith exact sequence A.1.1.

Proof. The statement is the Smith inequality applied to one of the involutions $T_{ \pm}$ in the double covering $Y \rightarrow X$ branched over $A$ (see 2.2.4). The left hand side here is $\beta_{*}\left(\mathbb{R} Y_{ \pm}\right)$, as it follows immediately from the Smith exact sequence of the deck translation involution, exact sequence of $(\mathbb{R} X, \mathbb{R} A)$, and Poincaré duality. The right hand side equals $\beta_{*}(Y)$. Indeed, from 2.2 .2 and the symmetry of the Betti numbers it follows that $\beta_{r}(Y)=\beta_{r}(X)$ for all $r \neq n$, and to find the remaining Betti number $\beta_{n}(Y)$ it suffices to compare the Euler characteristics (using, e.g., the Riemann-Hurwitz formula A.1.3(6)).

Remark. The left hand side of the inequality in 2.3 .2 above is equal to $\beta_{*}(\mathbb{R} A)+$ $2 \operatorname{dim} \operatorname{Ker}\left(\omega_{ \pm} \oplus \partial\right)$. In the case of projective hypersurfaces the inequality can be simplified, see 2.8.3; in this case $\omega_{ \pm}$is $k$-times the generator of $H^{1}\left(\mathbb{R} \mathbb{P}^{n}\right)$, where $2 k=\operatorname{deg} A$. (By a strange mistake, in the early papers on the subject another class was indicated.)

Unlike the case of curves, in general it is a difficult problem to estimate $\beta_{i}(\mathbb{R} X)$ (and even $\beta_{0}(\mathbb{R} X)$ ) separately; the sharp bound is not known even for surfaces of degree 5 in $\mathbb{P}^{3}$, see 3.5. Note also that 2.3 .1 and 2.3.2, when they both apply, give, in general, different restrictions to the topology of $(\mathbb{R} X, \mathbb{R} A)$. Certainly, the ampleness condition in 2.3.2 is only needed to evaluate the Betti numbers of $Y$; without it the right hand side of the inequality should be replaced with $\beta_{*}(Y)$.

According to 2.3.1, the difference $\beta_{*}(X)-\beta_{*}(\mathbb{R} X)$ is a nonnegative even integer. If this difference is $2 d$, one calls $X$ is an $(M-d)$-manifold (or conj an $(M-d)$ )involution). Similar to the case of curves and the classical Harnack inequality it is $M$ - (or close to $M$-) manifolds that satisfy certain additional congruence type prohibitions (see 2.7). 
2.4. Comessatti-Petrovsky-Oleinik inequalities. Recall that, if a compact complex manifold $X$ admits a Kähler metric (and this is the case for projective manifolds, since they inherit a Kähler metric from the ambient projective space), there is a canonical Hodge decomposition (Hodge structure)

$$
H^{r}(X, \mathbb{C})=\bigoplus_{p+q=r} H^{p, q}(X), \quad 0 \leqslant p, q \leqslant \operatorname{dim}_{\mathbb{C}} X,
$$

where $H^{p, q}(X)=H^{q}\left(X ; \Omega^{p}(X)\right)$ can be interpreted as the subspace of classes realized by $(p, q)$-forms. The Hodge numbers $h^{p, q}(X)=\operatorname{dim} H^{p, q}(X)$ are deformation invariants of $X$. A particular Kähler metric yields a further decomposition $H^{p, q}(X)=\bigoplus \Omega^{k} \wedge P^{p-k, q-k}(X)$, where $\Omega \in H^{1,1}(X)$ is the fundamental class of the metric and $P^{a, b} \subset H^{a, b}$ are the subspaces of so called primitive classes (see, e.g., [Ch]). If $X$ is real, it admits an invariant Kähler metric (e.g., obtained by the averaging) and for such a metric one has $\operatorname{conj}^{*} \Omega=-\Omega$.

The following result incorporates the Petrovsky [Pet], Petrovsky-Oleinik [PO], and Comessatti [Co1] inequalities (for double planes, projective hypersurfaces, and surfaces, respectively). For other generalizations of Petrovsky-Oleinik inequalities see [A2], [Kho1], [Kho2].

2.4.1. Theorem. If $X$ is a compact complex Kähler manifold with real structure, $\operatorname{dim} X=n=2 k$ even, then

$$
|\chi(\mathbb{R} X)-1| \leqslant h^{k, k}(X)-1 .
$$

If, besides, $A \subset X$ is an even real divisor, $A \sim 2 E$ for a real divisor $E$, then

$$
\begin{gathered}
\left|\chi\left(\mathbb{R} X_{-}\right)-\chi\left(\mathbb{R} X_{+}\right)\right| \leqslant h_{-}^{k, k}(Y), \quad \text { and } \\
\left|2 \chi\left(\mathbb{R} X_{ \pm}\right)-1\right| \leqslant h^{k, k}(Y)-1,
\end{gathered}
$$

where $Y=Y(E), \tau$ is the deck translation, and $h_{-}^{p q}(Y)=h^{p, q}(Y)-h^{p, q}(X)$ is the dimension of the $\tau$-skew-invariant part $H_{-}^{p q}(Y) \subset H^{p, q}(Y)$.

Proof. We prove the first assertion; the two others are similar. According to the Lefschetz fixed point theorem,

$$
\chi(\mathbb{R} X)=\sum_{r}(-1)^{r} \operatorname{Trace}\left(\operatorname{conj}^{*}, H^{r}(X)\right) .
$$

Since $\operatorname{conj}_{*} H^{p, q}(X)=H^{q, p}(X)$ and conj ${ }^{*} \Omega=-\Omega$ (for an invariant Kähler metric), in the decomposition of $H^{*}(X ; \mathbb{C})$ into primitive classes only the terms present in $H^{k, k}(X)=\sum \Omega^{j} \wedge P^{k-j, k-j}$ may contribute to $\chi(\mathbb{R} X)$ :

$$
\chi(\mathbb{R} X)=\sum_{j=0}^{k} \operatorname{Trace}\left(\text { conj }^{*}, P^{k-j, k-j}(X)\right) .
$$

It remains to observe that conj $^{*}=$ id on $P^{0,0}(X)$ and that $\mid$ Trace $\mid \leqslant \operatorname{dim}$.

The above inequalities are sharp for curves in $\mathbb{P}^{2}$, see [Pet], and for surfaces in $\mathbb{P}^{3}$, see [V2]; in these cases they are, respectively, the Petrovsky and Petrovsky-Oleinik inequalities. To our knowledge, in higher dimensions the question is still open. 
2.5. Hodge numbers. If $A$ is ample, the last two inequalities in 2.4 .1 can be made effective. Following F. Hirzebruch, consider the generalized Todd genus (or just $T_{y}$-genus $) T_{y}(X)=\sum(-1)^{q} h^{p, q}(X) y^{p}$. By the Hirzebruch-Riemann-Roch theorem [H2] $T_{y}(X)$ is equal to the value on $[X]$ of a certain degree $n$ polynomial $T_{n}\left(y ; c_{1}, \ldots, c_{n}\right)$ in the Chern classes $c_{i}=c_{i}(X)$. Furthermore, given $u_{1}, \ldots, u_{r} \in H^{2}(X)$, one can define the virtual Todd genus $T_{y}^{r}\left(u_{1}, \ldots, u_{r}\right)$, which is a polynomial in $c_{i}$ and $u_{j}$ with the following property: if the classes Poincaré dual to $u_{i}$ are realized by codimension 1 submanifolds $U_{i}$ intersecting transversally, then $T_{y}^{r}\left(u_{1}, \ldots, u_{r}\right)=T_{y}\left(U_{1} \cap \ldots \cap U_{r}\right)$.

The following statement is a consequence of 2.2 .2 (with $G=\mathbb{C}$ ):

2.5.1. Proposition (see [Kh2]). Let $A \subset X$ and $Y=Y(E)$ be as in 2.4.1. If $A$ is ample, then $h^{p, q}(Y)=h^{p, q}(X)$ for all $p, q$ with $p+q \neq n$ and

$$
\sum_{i=0}^{n}(-1)^{i}\left(h^{n-i, i}(Y)-h^{n-i, i}(X)\right) y^{i}=\sum_{r \geqslant 0} a_{r}(y) T_{y}^{r}(e, \ldots, e)_{X}-T_{y}(X),
$$

where $a_{r}(y)$ is the coefficient of $x^{r}$ in the formal power series expansion

$$
\frac{(1+y x)^{2}-(1-x)^{2}}{(1+y x)^{2}+y(1-x)^{2}} \cdot \frac{1}{x}=\sum_{r=0}^{\infty} a_{r}(y) x^{r}
$$

and $e=c_{1}(E)$. (The result does not depend on the choice of $E$ with $2 E \sim A$, as the calculation is done in the rational homology.)

Proof. From 2.2.2, the naturallity of the Hodge decomposition, and Serre duality it follows that $h^{p, q}(Y)=h^{p, q}(X)$ for $p+q \neq n$, and to find the remaining Hodge numbers in the middle dimension it suffices to know the Todd genus of $Y$, which, due to [Kh2], is given by $T_{y}(Y)=\sum_{r \geqslant 0} a_{r}(y) T_{y}^{r}(e, \ldots, e)_{X}$.

Explicit calculation for small dimensions gives the following values for $T_{y}(X)=$ $\sum_{i=0}^{n} T_{n, i} y^{i}($ where $n=\operatorname{dim} X)$ :

2.5.3. Corollary. Let $c_{i}=c_{i}(X)$. If $\operatorname{dim} X=2$, then

$$
\begin{gathered}
T_{2,0}=T_{2,2}=\frac{1}{12}\left(c_{1}^{2}+c_{2}\right)[X], \quad T_{2,1}=\frac{1}{6}\left(c_{1}^{2}-5 c_{2}\right)[X] \\
h^{1,1}(Y)=h^{1,1}(X)-T_{2,1}-\left(e c_{1}-3 e^{2}\right)[X] .
\end{gathered}
$$

If $\operatorname{dim} X=4$, then

$$
\begin{gathered}
T_{4,0}=T_{4,4}=\frac{1}{720}\left(-c_{1}^{4}+4 c_{1}^{2} c_{2}+3 c_{2}^{2}+c_{1} c_{3}-c_{4}\right)[X] \\
T_{4,1}=T_{4,3}=\frac{1}{180}\left(-c_{1}^{4}+4 c_{1}^{2} c_{2}+3 c_{2}^{2}-14 c_{1} c_{3}-31 c_{4}\right)[X], \\
T_{4,2}=\frac{1}{120}\left(-c_{1}^{4}+4 c_{1}^{2} c_{2}+3 c_{2}^{2}-19 c_{1} c_{3}+79 c_{4}\right)[X] \\
h^{2,2}(Y)=h^{2,2}(X)+T_{4,2}+ \\
\frac{1}{12}\left(115 e^{4}-46 e^{3} c_{1}-5 e^{2} c_{1}^{2}+31 e^{2} c_{2}+e c_{1} c_{2}-12 e c_{3}\right)[X] .
\end{gathered}
$$

If $X$ is a regular complete intersection in $\mathbb{P}^{N}$, its Hodge numbers and, hence, the bounds in 2.4 .1 can be found recursively. They depend only on the polydegree 
of $X$. Define polynomials $\chi_{s}^{q}\left(m_{1}, \ldots, m_{s} ; y\right)$ as follows:

$$
\begin{aligned}
& \chi_{0}^{0}(y)=1, \quad \chi_{s}^{0}\left(m_{1}, \ldots, m_{s} ; y\right)=0 \quad \text { for } s>0, \quad \text { and } \\
& \chi_{s}^{q}\left(\ldots, m_{s} ; y\right)=m_{s} \chi_{s-1}^{q-1}(\ldots ; y) \\
& +\sum_{\mu=1}^{m_{s}}\left[(y-1) \chi_{s}^{q-1}(\ldots, \mu-1 ; y)-y \chi_{s+1}^{q-1}(\ldots, \mu-1, \mu ; y)\right] \text {. }
\end{aligned}
$$

(Note that $\chi_{0}^{q}(y)=\sum_{r=0}^{q}(-1)^{r} y^{r}, \quad \chi_{q}^{q}\left(m_{1}, \ldots, m_{q} ; y\right)=m_{1} \ldots m_{q}$, and $\chi_{s}^{q}=0$ for $s>q$.)

2.5.5. Proposition. If a manifold $X$ of even dimension $n=2 l$ is a regular complete intersection in $\mathbb{P}^{n+s}$ of polydegree $\left(m_{1}, \ldots, m_{s}\right)$, then

$$
T_{y}(X)=\chi_{s}^{n+s}\left(m_{1}, \ldots, m_{s} ; y\right)
$$

In particular, $(-1)^{l} h^{l, l}(X)$ is equal to the coefficient of $y^{l}$ in $\chi_{s}^{n+s}\left(m_{1}, \ldots, m_{s} ; y\right)$.

If, further, $A \subset X$ is a submanifold cut on $X$ by a hypersurface of degree $2 k$ and $Y$ is the double covering of $X$ branched over $A$, then

$$
T_{y}(Y)=\sum_{r=0}^{n} a_{r}(y) \chi_{r+s}^{n+s}\left(m_{1}, \ldots, m_{s}, k, \ldots, k ; y\right)
$$

(See (2.5.2) for the definition of $a_{r}(y)$.) In particular, $(-1)^{l} h^{l, l}(Y)$ is equal to the coefficient of $y^{l}$ in the above polynomial.

Proof (see $[\mathrm{Kh} 2])$. The statement is an immediate consequence of the functional equation for $T_{y}$-genus [H2, Theorem 11.3.1] and the well known fact that $h^{p, q}(X)=$ 0 unless $p=q$ or $p+q=n$.

In small dimensions (surfaces in $\mathbb{P}^{q}$ and hypersurfaces in $\mathbb{P}^{5}$ ) Proposition 2.5.5 gives the following (to simplify the formulas we denote by $\mu_{i}$ the $i$-th elementary symmetric polynomial in $m_{1}, \ldots, m_{s}$; certainly, $\mu_{0}=1$ and $\mu_{i}=0$ for $i>s$ ).

2.5.6. Corollary. If $X \subset \mathbb{P}^{q}, \operatorname{dim} X=2$, then

$$
\begin{gathered}
h^{1,1}(X)=\frac{1}{12} \mu_{q-2}\left(8 \mu_{1}^{2}-10 \mu_{2}-6(q+1) \mu_{1}+(q+1)(3 q-2)\right), \\
h^{1,1}(Y)=\mu_{q-2} k\left(3 k+\mu_{1}-(q+1)\right)+2 h^{1,1}(X)
\end{gathered}
$$

If $X \subset \mathbb{P}^{5}, \operatorname{dim} X=4$, then

$$
\begin{aligned}
& s=0: \quad h^{2,2}(Y)=\frac{115}{12} k^{4}-\frac{115}{6} k^{3}+\frac{185}{12} k^{2}-\frac{35}{6} k+2, \\
& s=1: \quad h^{2,2}(X)=\frac{11}{20} m_{1}^{5}-\frac{11}{4} m_{1}^{4}+\frac{23}{4} m_{1}^{3}-\frac{25}{4} m_{1}^{2}+\frac{37}{10} m_{1} \text {, } \\
& h^{2,2}(Y)=\frac{115}{12} m_{1} k^{4}+\left(\frac{23}{6} m_{1}-23\right) m_{1} k^{3} \\
& +\left(\frac{13}{6} m_{1}^{2}-\frac{21}{2} m_{1}+\frac{95}{4}\right) m_{1} k^{2} \\
& +\left(\frac{11}{12} m_{1}^{3}-5 m_{1}^{2}+\frac{43}{4} m_{1}-\frac{25}{2}\right) m_{1} k+2 h^{2,2}(X) \text {. }
\end{aligned}
$$

Remark. Originally the bound in the Petrovsky-Oleinik inequality was expressed via the number of certain monomials rather than in terms of the Hodge structure. 
The coincidence of the two bounds was proved by V. Zvonilov [Zv1] using 2.5.1. It is closely related to the following beautiful rule: if $X \subset \mathbb{P}^{2 k+1}$ is a hypersurface, $\operatorname{deg} X=d$, then $h^{k, k}(X)-1$ is the number of the integral points in the layer $k d<\sum\left(x_{i}+1\right)<(k+1) d$ of the cube $[0, d-2]^{2 k+1} \subset \mathbb{R}^{2 k+1}$. Other layers of the cube give the other Hodge numbers. The formula extends to hypersurfaces in spaces of even dimension. Relations of this type were first observed by, probably, J. Steenbrink [St], who found an explicit monomial basis for the vanishing Hodge structure of a semiquasihomogeneous singularity. For further generalizations see [DKh] and [Va2].

2.6. Types of involutions. In the theory of real curves the notion of separating (or type I, see Introduction) curve is crucial. In higher dimensions, when $\mathbb{R} X$ has (real) codimension greater than one, it cannot separate $X$, but the homology vanishing condition, $[\mathbb{R} X]=0 \in H_{n}(X)$, still makes sense. In various applications the properties of $\mathbb{R} X$ may depend on whether it is homologous to a certain distinguished class $u \in H_{n}(X)$ which is usually natural (say, a characteristic class of $X$ ), but does not need to be zero. Thus, given a class $u \in H_{n}(X)$, we say that the real structure conj on $X$ (or just $X$ itself, or $\mathbb{R} X$ ) is of type $\mathrm{I}_{u}$ if $[\mathbb{R} X]$ is homologous to $u$. Here are the special cases used most commonly:

(1) $\mathrm{I}_{0}$ or $\mathrm{I}_{\mathrm{abs}}$, with $u=0$. Type $\mathrm{I}_{0}$ is sometimes called the absolute type $\mathrm{I}$;

(2) $\mathrm{I}_{\mathrm{wu}}$, where $u=u_{n}(X)$ is the $n$-th Wu class of $X$. (Recall that $u_{n}(X)$ is the characteristic element of the intersection form of $X$. Due to the Wu formula, $u_{n}(X)$ is a certain polynomial in the Stiefel-Whitney classes of $\left.X^{3}\right)$;

(3) $\mathrm{I}_{\mathrm{hp}}$, with $u$ equal to the $(n / 2)$-th power of the hyperplane section. (Certainly, this only makes sense for a fixed embedding $X \hookrightarrow \mathbb{P}^{N}$.)

In the case of projective varieties the notion of types $\mathrm{I}_{0}$ and $\mathrm{I}_{\mathrm{hp}}$ appeared in [Kh5]. In [V1] types $\mathrm{I}_{0}$ and $\mathrm{I}_{\mathrm{hp}}$ are called $\mathrm{I}_{\mathrm{abs}}$ and $\mathrm{I}_{\text {rel }}$ respectively, and a hypersurface which is not of one of these types is said to have type II. However, this terminology does not seem commonly accepted. Usually, depending on a particular problem, it is reasonable to distinguish a class $u \in H_{n}(X)$ and consider manifolds of types $\mathrm{I}_{\text {abs }}$ and $\mathrm{I}_{u}=\mathrm{I}_{\text {rel }}$, regarding the rest as type II. E.g., in most results below it is type $\mathrm{I}_{\mathrm{wu}}$ that plays essential rôle.

2.7. Congruences. Next two theorems are direct generalizations of Gudkov, Arnol'd, Rokhlin, Kharlamov, and Krakhnov congruences.

2.7.1. Extremal congruences. Let $X$ be an even dimensional $(M-d)$-manifold with real structure. Then:

(1) if $d=0$, then $\chi(\mathbb{R} X)=\sigma(X) \bmod 16$;

(2) if $d=1$, then $\chi(\mathbb{R} X)=\sigma(X) \pm 2 \bmod 16$;

(3) if $d=2$ and $\chi(\mathbb{R} X)=\sigma(X)+8 \bmod 16$, then $X$ is of type $\mathrm{I}_{\mathrm{wu}}$.

2.7.2. Generalized Arnol'd congruence. Let $X$ be a manifold with real structure of type $\mathrm{I}_{\mathrm{wu}}$. If $\operatorname{dim} X$ is even, then $\chi(\mathbb{R} X)=\sigma(X) \bmod 8$.

2.7.3. Proposition. An $M$-manifold with real structure is of type $\mathrm{I}_{\mathrm{wu}}$.

The signature of a complex analytic manifold $X$ equals $T_{1}(X)$ (see 2.4). If $X$ is a surface, this gives $\sigma(X)=\frac{1}{3}\left(c_{1}^{2}-2 c_{2}\right)[X]$. If $X$ is a regular complete intersection

\footnotetext{
${ }^{3}$ Since $X$ is a complex manifold, $w_{2 i+1}(X)=0$ and $w_{2 i}(X)=c_{i}(X) \bmod 2$. Note that $\operatorname{conj}_{*} c_{i}=(-1)^{i} c_{i}$; in particular, all the $\bmod 2$ characteristic classes of $X$ are $\operatorname{conj}_{*}$-invariant.
} 
in $\mathbb{P}^{q}$, its signature can be found using Proposition 2.5.5 and (2.5.4). Here are some partial results:

2.7.4. Signature of a complete intersection. Let $X$ be a regular complete intersection in $\mathbb{P}^{q}$ of polydegree $\left(m_{1}, \ldots, m_{s}\right)$ and even dimension $n=q-s$.

(1) If $n=2$, then $\sigma(X)=-\frac{1}{3} \mu_{q-2}\left(\mu_{1}^{2}-2 \mu_{2}-q-1\right)$, where $\mu_{i}$ is the $i$-th elementary symmetric polynomial in $\left(m_{1}, \ldots, m_{s}\right)$.

(2) If $s=q+2 \bmod 4$ and $m_{1}+\cdots+m_{s}=q+1 \bmod 2$, then $\sigma(X)=0 \bmod 16$.

(3) If $s=q \bmod 4$ and all $m_{i}$ but, maybe, one (say, $m_{s}$ ) are odd, then $\sigma(X)=$ $m_{1} \ldots m_{s} \bmod 16$.

(4) If $X$ is a hypersurface (i.e., $s=1$ ), then

$\sigma(X)=m_{1}(\bmod 16)$ if $n=0 \bmod 4$

$\sigma(X)=0 \bmod 16$ if $n=2 \bmod 4$ and $m_{1}$ is even;

$\sigma(X)=1-m_{1}\left(m_{1}-1\right) \bmod 16$ if $n=2 \bmod 4$ and $m_{1}$ is odd.

Proof. We use the identity $\sigma(X)=\chi_{s}^{q}\left(m_{1}, \ldots, m_{s} ; 1\right)$. Statement (1) is proved by induction using (2.5.4). Statement (2) follows from the fact that the signature of a $(8 k+4)$-dimensional Spin-manifold is divisible by 16 (see [Och]). To prove (3), we proceed by induction in $s$ and $m_{s}$ and use the functional equation for $T_{y}$-genus [H2, Theorem 11.3.1)], which implies

$$
\chi_{s}^{q}\left(\ldots, m_{s} ; 1\right)=\chi_{s}^{q}\left(\ldots, m_{s}-1 ; 1\right)+\chi_{s-1}^{q-1}(\ldots ; 1)-\chi_{s+1}^{q-1}\left(\ldots, m_{s}-1, m_{s} ; 1\right) .
$$

Under the hypotheses of (3) the last term in (2.7.5) vanishes due to (2).

Statements (2) and (3) cover the first two cases in (4). In the last case we use $(2.7 .5)$ again; now $\chi_{1}^{q}(m-1 ; 1)=0 \bmod 16$ due to $(2), \chi_{0}^{q-1}(1)=\sigma\left(\mathbb{P}^{q-1}\right)=1$, and $\chi_{2}^{q-1}(m-1, m ; 1)=(m-1) m \bmod 16$ due to $(3)$.

The proofs of 2.7.1-2.7.3 are similar to each other; the key ingredient is Lemma 2.7.6 below.

Let $n=\operatorname{dim} X$. Denote $H=H_{n}(X ; \mathbb{Z}) /$ Tors and let $H^{ \pm 1} \subset H$ be the eigensubgroups of conj $_{*}$ and $J=H /\left(H^{+1} \oplus H^{-1}\right)$. Denote by $B: H \otimes H \rightarrow \mathbb{Z}$ the intersection form $(x, y) \mapsto x \circ y$ of $X$, and by $B^{\text {conj }}$, the twisted intersection form $(x, y) \mapsto x \circ \operatorname{conj}_{*} y$, see A.3. Let $q^{ \pm}$be the discriminant quadratic space associated with $\left.B\right|_{H^{ \pm}}$. As is known (see B.3), $J$ and $q^{ \pm}$are $\mathbb{Z}_{2}$-vector spaces isomorphic as groups.

2.7.6. Lemma. Let, as above, $X$ be an $(M-d)$-manifold with real structure, $\operatorname{dim} X=n$. Then:

(1) the image of $[\mathbb{R} X]$ in $H_{2}(X)$ is the characteristic element of the twisted intersection form $B^{\text {conj }}$

(2) both $u_{n}(X)$ and $[\mathbb{R} X] \in H_{n}(X)$ are integral classes, i.e., they belong to the image of $H_{n}(X ; \mathbb{Z})$;

(3) $d \geqslant \operatorname{dim} \operatorname{Coker}\left[\left(1+\operatorname{conj}_{*}\right): H_{*}(X) \rightarrow H_{*}(X)\right] \geqslant \operatorname{dim} J$.

Let, besides, $n=\operatorname{dim} X$ be even, $n=2 k$. Denote $\epsilon=(-1)^{k+1}$. Then

(4) $\chi(\mathbb{R} X)=(-1)^{k+1} \sigma\left(B^{\text {conj }}\right)=\sigma(X)-2 \sigma\left(H^{\epsilon}\right)$;

(5) $H^{\epsilon}$ is an even lattice;

(6) $\operatorname{dim} J=d \bmod 2$. 
Proof. Statement (1) is the Arnol'd lemma A.3.3. (4) is an immediate consequence of A.3.2, and (5) follows from the fact that $u_{n}(X)$, like any characteristic class of $X$, is realized by a formal linear combination of real cycles of dimension $k$ (see, e.g., [R5]) and, hence, belongs to $H^{-\epsilon}$.

To prove (2) note that both the standard and twisted $\mathbb{Z}_{2}$-valued intersection forms vanish on the image of Tors $H_{n}(X ; \mathbb{Z})$ (as so do their $\mathbb{Z}$-valued counterparts). Hence, their characteristic classes annihilate Tors $H_{n}(X ; \mathbb{Z})$ and thus are integral.

Statement (3) follows from A.1.3(1) and the construction of the Smith exact sequence given in A.1.5. Indeed, $\mathrm{Ker} \mathrm{pr}_{*}$ in A.1.1 consists of geometrically invariant, and hence conj $j_{*}$-invariant cycles, which must belong to $\operatorname{Ker}\left(1+\operatorname{conj}_{*}\right)$. This gives the first inequality in (3); the second one is obvious, as $\operatorname{dim} J$ equals $\operatorname{dim} \operatorname{Ker}(1+$ $\mathrm{conj}_{*}$ ) restricted to $H \otimes \mathbb{Z}_{2}$.

The proof of (6) is based on the following two facts:

2.7.7. Lemma. For any closed manifold $M$ of even real dimension $n=2 k$ one has $\beta_{*}(M)=(-1)^{k} \chi(M) \bmod 4$.

Proof. If $k=2 l$ is even, by Poincaré duality $\beta_{*}(M)-\chi(M)=2 \sum_{r=0}^{k-1} \beta_{2 r+1}(M)=$ $4 \sum_{r=0}^{l-1} \beta_{2 r+1}(M)$. The proof for $k$ odd is similar.

2.7.8. Lemma. For any closed complex manifold $X$ of even complex dimension $n=2 k$ one has $\sigma(X)=(-1)^{k} \chi(X) \bmod 4$.

Proof. The proof is similar to the previous one, using the $T_{y}$-characteristic (see 2.4), the identities $\sigma(X)=T_{1}(X)$ and $\chi(X)=T_{-1}(X)$, and the symmetry $T_{n, i}=$ $(-1)^{n} T_{n, n-i}$ of the coefficients $T_{n, i}$ in $T_{y}(X)=\sum T_{n, i} y^{i}$ (see [H2, Section 1.8]; note that $T_{n, i}$ take integral values on any, not necessarily Kähler, complex manifold.)

Lemmas 2.7.7 and 2.7.8 applied to $X$ give $\beta_{*}(X)=(-1)^{k} \sigma(X) \bmod 4$. Then (4) turns into $(-1)^{k} \beta_{*}(\mathbb{R} X)=(-1)^{k} \beta_{*}(X)-2 \sigma\left(H^{\epsilon}\right) \bmod 4$, and (6) follows from the congruence $\sigma\left(H^{\epsilon}\right)=\operatorname{dim} q^{\epsilon} \bmod 2$ (the lattice is even due to (5)) and the identity $\operatorname{dim} J=\operatorname{dim} q^{ \pm}$.

Remark. Lemma 2.7.8 extends to almost complex manifolds; one can use either Atiyah-Dupont formula [AD] (see G. Wilson [Wi]) or cobordism arguments.

Proof of Theorem 2.7.1. Under the hypotheses of 2.7.1(1) or (2) from 2.7.6(3) and (6) it follows that $\operatorname{dim} q^{\epsilon}=\operatorname{dim} J=0$ or 1 , respectively (where still $\epsilon=$ $(-1)^{k+1}$ ), and Theorem B.2.2 applied to $H^{\epsilon}$ in 2.7.6(4) gives 2.7.1(1) and (2).

Similar arguments show that under the hypotheses of 2.7.1(3) the discriminant forms $q^{ \pm}$, whose dimensions are at most 2, are even and, due to B.3.1, the difference $[\mathbb{R} X]-u_{n}(X)$ annihilates all the integral classes in $H_{n}(X)$, i.e., belongs to the image of Tors $H_{n}(X ; \mathbb{Z})$. Now, using again 2.7.6(3), where both the inequalities turn into equalities, one concludes that each element of $H_{n}(X) / H_{n}(X ; \mathbb{Z}) \otimes \mathbb{Z}_{2}$ has a conj $j_{*}$-invariant representative. Hence, $B$ and $B^{\text {conj }}$ coincide on such classes and $u_{n}(X)-[\mathbb{R} X]$ vanish.

Proof of Theorem 2.7.2. Since $[\mathbb{R} X]$ and $u_{n}(X)$ coincide, from B.3.1 it follows that $q^{ \pm}$are even discriminant lattices. Hence, $\operatorname{Br} q^{ \pm}=0 \bmod 4$, and 2.7.6(4) applies.

Proof of Proposition 2.7.3. The statement follows from 2.7.6(1) and 2.7.6(3), which implies that conj $_{*}$ acts as identity on $H_{*}(X)$.

\subsection{Additional prohibitions.}


2.8.1. Generalized Arnol'd inequality. Let $X$ be a closed complex Kähler manifold with real structure and $\operatorname{dim} X=n=2 k$ even. Then the number $p_{-}$of the orientable components of $\mathbb{R} X$ with negative Euler characteristic satisfies the inequality

$$
p_{-} \leqslant \frac{1}{4}\left(b_{n}(X)-(-1)^{k} \sigma(X)\right)+\frac{1}{2} \sum_{j=1}^{k}(-1)^{j} h^{k-j, k-j}(X)+\frac{1}{2}\left((-1)^{k}-1\right) .
$$

Proof. We proceed exactly as in [A1] and derive the inequality from $p_{-} \leqslant \sigma_{\epsilon}^{+1}$, where $\epsilon=(-1)^{k+1}$ and $\sigma_{ \pm}^{+1}$ are the inertia indices of the intersection form of $X$ restricted to $H_{n}^{+1}(X ; \mathbb{Z})$. In order to find $\sigma_{\epsilon}^{+1}$, we use the relations

$$
\begin{aligned}
& \sigma_{+}^{+1}+\sigma_{-}^{+1}+\sigma_{+}^{-1}+\sigma_{-}^{-1}=b^{n}(X), \\
& \sigma_{+}^{+1}-\sigma_{-}^{+1}+\sigma_{+}^{-1}-\sigma_{-}^{-1}=\sigma(X), \\
& \sigma_{+}^{+1}-\sigma_{-}^{+1}-\sigma_{+}^{-1}+\sigma_{-}^{-1}=(-1)^{k} \chi(\mathbb{R} X), \\
& \sigma_{+}^{+1}+\sigma_{-}^{+1}-\sigma_{+}^{-1}-\sigma_{-}^{-1}=\operatorname{Trace}\left(\operatorname{conj}^{*}, H^{n}(X ; \mathbb{Z})\right) .
\end{aligned}
$$

In the right hand side of the last equation $H^{n}(X ; \mathbb{Z})$ can be replaced with $H^{k, k}(X)$, and an estimate on $\sigma_{\epsilon}^{+1}$ follows from comparing

$$
\operatorname{Trace}\left(\operatorname{conj}^{*}, H^{k, k}(X)\right)=\sum_{j=0}^{k}(-1)^{j} \operatorname{Trace}\left(\operatorname{conj}^{*}, P^{k-j, k-j}(X)\right)
$$

with (2.4.2) and the obvious inequality

$$
\text { - Trace }\left(\operatorname{conj}^{*}, P^{i, i}(X)\right) \leqslant \operatorname{dim} P^{i, i}(X)=h^{i, i}(X)-h^{i-1, i-1}(X) .
$$

(In the case of $k$ odd one can also use the fact that - Trace $\left(\right.$ conj $\left.^{*}, P^{0,0}(X)\right)$ contributes to $\sigma_{\epsilon}^{+1}$ and, on the other hand, conj ${ }^{*}=\mathrm{id}$ on $P^{0,0}(X)$.)

2.8.2. For a subset $S \subset \mathbb{R} \mathbb{P}^{N}$ let

$$
\ell(S)=\max \left\{-1, i \mid \text { in }^{*}: H^{i}\left(\mathbb{R} \mathbb{P}^{N}\right) \rightarrow H^{i}(S) \text { is nontrivial }\right\},
$$

where in: $S \hookrightarrow \mathbb{R} \mathbb{P}^{N}$ is the inclusion. If $A \subset \mathbb{P}^{N}$ is a real projective variety, we let $\ell(A)=\ell(\mathbb{R} A)$. It is clear that $\operatorname{in}^{*}: H^{i}\left(\mathbb{R} \mathbb{P}^{N}\right) \rightarrow H^{i}(\mathbb{R} A)$ is a nontrivial homomorphism for all $i \leqslant \ell(A)$ and that $\ell(A)=n=\operatorname{dim} A$ if $\operatorname{deg} A$ is odd. (Recall that the degree of $A$ is the number of intersection points of $A$ and a generic $(N-n)$-plane in $\mathbb{P}^{N}$.) The following simple statement is a direct consequence of the Poincare duality and the standard exact sequences.

2.8.3. Proposition. If $\operatorname{deg} A$ is even, then $\ell\left(\mathbb{R P}_{+}\right)=\ell(A) \leqslant \ell\left(\mathbb{R P}_{-}\right)=n-l(A)$ and, in particular, $\ell(A) \leqslant \frac{n}{2}$.

Remark. As it follows from 2.8.3 and the remark after 2.3.2, if $\operatorname{deg} A$ is even, the double covering of $\mathbb{P}^{n+1}$ is an $M$-variety (under a proper choice of the covering real structure) if and only if $A$ is an $(M-d)$-variety, where for $n$ even

$$
d= \begin{cases}n-2 \ell(\mathbb{R} A), & \text { if } m=0 \bmod 4, \\ 1, & \text { if } m=2 \bmod 4 \text { and } n>2 \ell(\mathbb{R} A), \text { or } \\ 0, & \text { if } m=2 \bmod 4 \text { and } n=2 \ell(\mathbb{R} A) .\end{cases}
$$


and for $n$ odd

$$
d= \begin{cases}n-2 \ell(\mathbb{R} A)-1, & \text { if } m=0 \bmod 4, \\ 0, & \text { if } m=2 \bmod 4 .\end{cases}
$$

Another, related, consequence of the same calculation is an improvement of the Smith-Thom bound: $A$ can not be an $(M-l)$-variety with $l<d$ given above.

The invariant $\ell(\mathbb{R} A)$ was introduced in $[\mathrm{Kh} 5]$; the results of $[\mathrm{Kh} 5]$ were later improved by I. Kalinin [Ka].

2.8.4. Additional extremal congruences for $(M-d)$-hypersurfaces. Let $A \subset \mathbb{P}^{n+1}$ be a real $(M-d)$-hypersurface of even dimension $n$ and degree $m$. If $m= \pm 2 \bmod 8$ and $n / 2-\ell(A)$ is odd, then

(1) if $d=n / 2-\ell(A)$, then $\chi(\mathbb{R} A)=\sigma(A) \mp 2 \bmod 16$;

(2) If $d=n / 2-\ell(A)+1$, then $\chi(\mathbb{R} A)=\sigma(A), \sigma(A) \mp 4 \bmod 16$.

If $m=0 \bmod 8$ and $\ell(A)<n / 2$, then

(2) if $d=n-2 \ell(A)$, then $\chi(\mathbb{R} A)=\sigma(A) \bmod 16$;

(3) if $d=n-2 \ell(A)+1$, then $\chi(\mathbb{R} A)=\sigma(A) \pm 2 \bmod 16$.

Proof of Theorem 2.8.4 (see [Ka]). Without going too deep into the details, we just outline the principal ideas. Like the other extremal congruences, 2.8.4 is derived from 2.7.6(4) and (5) using Theorem B.2.2. The crucial point, which replaces 2.7.6(3) and (6) and gives an estimate on $\operatorname{dim} J=\operatorname{dim} q^{ \pm}$, is the following lemma:

2.8.5. Lemma (see $[\mathrm{Ka}])$. Let $A \subset \mathbb{P}^{n+1}$ be a real $(M-d)$-hypersurface of dimension $n$ and degree $m$.

(1) If $\ell(A) \geqslant[(n-1) / 2]$, then $\operatorname{dim} J=d$.

(2) If $\ell(A)<[(n-1) / 2]$, then

$$
\operatorname{dim} J=d- \begin{cases}2([(n-1) / 2]-\ell(A)) & \text { if } m=0 \bmod 4, \\ 2[(n / 2-\ell(A)) / 2] & \text { if } m=2 \bmod 4 \text { and } n \text { is even, } \\ (n-1) / 2-\ell(A) & \text { if } m=2 \bmod 4 \text { and } n \text { is odd. }\end{cases}
$$

(Here $[x]$ denotes the integral part of $x$.)

Lemma 2.8.5 is proved using Kalinin's spectral sequence $\left({ }^{r} E^{*},{ }^{r} d^{*}\right)$, see A.2. If $A$ is a regular complete intersection in $\mathbb{P}^{N}$, the difference $d-\operatorname{dim} J$ is equal to the number of nontrivial differentials ${ }^{r} d^{i}$ with $r>1$. Lemma 2.8.5 is obtained in [Ka] from an explicit calculation of ${ }^{r} E^{*}\left(\mathbb{P}^{N}\right)$ and ${ }^{r} E^{*}\left(\mathbb{P}^{n+1} \backslash A\right)$. This calculation also gives the other key ingredient of the proof of 2.8.4, which is stated below. Denote by $h$ the generator of $H^{2}\left(\mathbb{P}^{N}\right)$ and let $\delta_{h}(A)=0$ if $n=\operatorname{dim} A$ is odd or $n$ is even and in $^{*} h^{n / 2} \in \operatorname{Im}\left(1+\right.$ conj $\left.^{*}\right)$ in $H^{n}\left(A ; \mathbb{Z}_{2}\right)$, and $\delta_{h}(A)=1$ otherwise.

2.8.6. Lemma (see $[\mathrm{Ka}]$ ). Let $A \subset \mathbb{P}^{n+1}$ be a real hypersurface of even dimension $n$ and degree $m$. If $m=2 \bmod 4$, then

$$
\delta_{h}(A)= \begin{cases}0 & \text { if } \ell(A)<n / 2 \text { and } \ell(A)=n / 2-1 \bmod 2, \\ 1 & \text { otherwise }\end{cases}
$$

if $m=0 \bmod 4$, then

$$
\delta_{h}(A)= \begin{cases}0 & \text { if } \ell(A)<n / 2 \\ 1 & \text { if } \ell(A) \geqslant n / 2 .\end{cases}
$$


From the lemma it follows that under the hypotheses of 2.8 .4 one has in ${ }^{*} h^{n / 2} \in$ $\operatorname{Im}\left(1+\right.$ conj $\left.^{*}\right)$. This gives additional information on $\operatorname{Br} q^{ \pm}$when $\operatorname{dim} q^{ \pm}=\operatorname{dim} J$ is small (see B.1.1).

2.9. Inherited structures on the real part. In the rest of this section we briefly discuss a few constructions generalizing, to an extent, the notion of complex orientation of a dividing real curve.

2.9.1. Spin-orientations. Let $X$ be a manifold with real structure conj. Than any conj-invariant Spin-structure on $X$ defines, in a natural way, a semi-orientation (i.e., pair of opposite orientations) on $\mathbb{R} X$.

Remark. If $X$ is Spin and $H_{1}(X)=0$, the only Spin-structure on $X$ is obviously conj-invariant and, hence, $\mathbb{R} X$ has a canonical semi-orientation. In particular, it is orientable (cf. 3.4.2). The orientability statement extends to involutions on arbitrary manifolds and is known as Edmonds theorem [Ed].

Construction. One needs to compare orientations at two points $x_{1}, x_{2} \in \mathbb{R} X$. Connect $x_{1}, x_{2}$ by a path $\gamma$ in $X$, represent the two orientations by tangent $n$-frames $\Xi_{1}, \Xi_{2}\left(\right.$ where $\left.n=\operatorname{dim}_{\mathbb{C}} X=\operatorname{dim}_{\mathbb{R}} \mathbb{R} X\right)$, extend $\left(\Xi_{1}, \sqrt{-1} \Xi_{1}\right)$ and $\left(\Xi_{2}, \sqrt{-1} \Xi_{2}\right)$ to a $2 n$-frame field $\left(\xi_{1}, \ldots, \xi_{2 n}\right)$ on $\gamma$, and evaluate the chosen Spin-structure on the loop $\gamma \circ \operatorname{conj}_{*} \gamma$, where conj $_{*} \gamma$ is framed with

$$
\left(\text { conj }^{*} \xi_{1}, \ldots, \text { conj }^{*} \xi_{n},- \text { conj }^{*} \xi_{n+1}, \ldots,- \text { conj }^{*} \xi_{2 n}\right) .
$$

The two orientations are regarded coherent if the resulting value is 0 . It is straightforward to check that, if the Spin-structure is conj-invariant, the result does not depend on the choices made.

2.9.2. Stiefel orientations. Recall that an orientation of a smooth manifold $Y$ can be defined as a homotopy class of lifts to $B S O$ of a classifying map $f_{Y}: Y \rightarrow B O$ of the tangent bundle of $Y$. The fibration $B S O \rightarrow B O$ can, in turn, be regarded as the $K\left(\mathbb{Z}_{2}, 0\right)$ fibration killing $w_{1} \in H^{1}(B O)$. Generalizing this approach one can fix a characteristic class $\omega \in H^{i+1}(B O)$ and define $\omega$-structures on $Y$ as homotopy classes of lifts of $f_{Y}$ to a $K\left(\mathbb{Z}_{2}, i\right)$-fibration $B O_{\omega} \rightarrow B O$ killing $\omega$. It is easy to see that $Y$ admits an $\omega$-structure if and only if $\omega(Y)=0$ and, if nonempty, the set of $\omega$-structures on $Y$ is an affine space over $H^{i}(Y)$ (see, e.g., [Deg2]). Thus, orientations and Spin- (more precisely, $\mathrm{Pin}^{+}{ }^{-}$) structures are, respectively, $w_{1}$ - and $w_{2}$-structures.

If $\omega=w_{i+1}$ is a Stiefel-Whitney class, one can replace $B O_{\omega}$ with the corresponding associated Stiefel bundle and show that a $w_{i}$-structure can be regarded as a $\mathbb{Z}_{2}$-valued function on the homotopy classes of $(n-i)$-framed $i$-cycles (where $n=\operatorname{dim} Y)$. This gives rise to the following generalization of the notion of complex orientation of a dividing curve (see [V4] or, for a more formal approach, [Deg1]). Let $X$ be a complex manifold with real structure, $\operatorname{dim} X=n$, and $[\mathbb{R} X]$ vanishes in $H_{n}(X)$. Then $\mathbb{R} X$ possesses a canonical $w_{n}$-structure, whose value on a 1 -framed $(n-1)$-cycle $\gamma$ is defined as the linking coefficient of $\mathbb{R} X$ and a shift of $\gamma$ along the framing multiplied by $\sqrt{-1}$. (More precisely, this is a partial structure, defined on the kernel of the inclusion homomorphism $H_{n-1}(\mathbb{R} X) \rightarrow H_{n-1}(X)$.) The assumption $[\mathbb{R} X]=0$ assures that the linking coefficient is well defined. The construction admits a further generalization. Let $\gamma$ be an $(n-i)$-cycle and $\left(\xi_{1}, \ldots, \xi_{i}\right)$ its framing. Proceed as in the geometric construction of Viro homomorphisms (see A.2) 
and include $\gamma$ into an $(n-1)$-cycle $\gamma^{\prime}$ in $X$ tangent to $\sqrt{-1} \xi_{1}, \ldots, \sqrt{-1} \xi_{i-1}$; then shift $\gamma^{\prime}$ along an extension of $\sqrt{-1} \xi_{i}$ and evaluate the linking coefficient of the shift and $\mathbb{R} X$. A detailed analysis shows that this construction gives rise to a partial $w_{n-i+1}$-structure on $\mathbb{R} X$, defined on the kernel of bv $b_{n-1}: H_{n-i}(\mathbb{R} X) \rightarrow{ }^{i} E_{n-1}(X)$, provided that $\mathrm{bv}_{n+i-1}[\mathbb{R} X]$ vanishes in ${ }^{i} E_{n+i-1}(X)$. In particular, the last condition implies that $w_{n}(X)=\ldots=w_{n-i+1}(X)=0$.

\section{Surfaces}

3.1. Basic results. In this section by complex surface we mean a closed complex analytic manifold of complex dimension 2. First, we restate some results of Section 2 in more topological terms.

3.1.1. Theorem. Let $X$ be a complex surface with real structure. Then

$$
\beta_{*}(\mathbb{R} X) \leqslant \beta_{*}(X) \quad \text { and } \quad \beta_{*}(\mathbb{R} X)=\beta_{*}(X) \bmod 2
$$

Let, further, $A$ be a nonsingular even ample real divisor on $X$ and $Y \rightarrow X$ a double covering branched over $A$. Then

$$
\begin{aligned}
& \beta_{*}\left(\mathbb{R} X_{-}\right)+\beta_{*}\left(\mathbb{R} X_{+}\right) \leqslant \beta_{2}(X)+\beta_{*}(A) \text { and } \\
& 2 \beta_{*}\left(\mathbb{R} X_{ \pm}\right)-4 c_{0}\left(\mathbb{R} X_{ \pm}\right) \leqslant 2 \beta_{2}(X)+\beta_{*}(A),
\end{aligned}
$$

where $\mathbb{R} X_{ \pm}$are the two halves of $\mathbb{R} X \backslash \mathbb{R} A$ (see 2.2) and $c_{0}\left(\mathbb{R} X_{ \pm}\right)$is the number of closed components of $\mathbb{R} X_{ \pm}$covered nontrivially in $\mathbb{R} Y_{ \pm}$.

According to the Nakai-Moishezon criterion (see, e.g., [BPV]) an effective divisor $A$ on a nonsingular Kähler surface $X$ is ample if and only if $A \circ D>0$ for any irreducible curve $D \subset X$. In general, the ampleness condition in 3.1 .1 can be replaced with a weaker requirement that $A$ should be connected and the inclusion homomorphism $H_{1}(A) \rightarrow H_{1}(X)$ should be onto. Under these hypotheses from the Smith exact sequence it follows that $\beta_{1}(Y)=\beta_{1}(X)$.

3.1.2. Theorem. Let $X$ be a complex Kähler surface with real structure. Then

$$
|\chi(\mathbb{R} X)-1| \leqslant \sigma^{-}(X)
$$

where $\sigma^{-}(X)=\frac{1}{2}\left[b_{2}(X)-\sigma(X)\right]$ is the negative inertia index of the intersection form of $X$. Let, further, $A \subset X$ be a nonsingular ample even real divisor on $X$ so that there is a real divisor $E$ on $X$ with $2 E \sim A$. Then

$$
\begin{gathered}
\left|\chi\left(\mathbb{R} X_{-}\right)-\chi\left(\mathbb{R} X_{+}\right)\right| \leqslant \sigma^{-}(X)-b_{1}(X)+1+\frac{1}{4}[A]^{2}-\frac{1}{2} \chi(A) \\
\left|2 \chi\left(\mathbb{R} X_{ \pm}\right)-1\right| \leqslant 2 \sigma^{-}(X)-b_{1}(X)+1+\frac{1}{4}[A]^{2}-\frac{1}{2} \chi(A)
\end{gathered}
$$

Note that here, as well as in many other statements about surfaces (at least in this survey) the Kähler property is only used to assert that conj $j_{*}$ is traceless on $H_{1}(X ; \mathbb{R})$. If $X$ is not Kähler (but still complex analytic), there still is a canonical subspace $H^{1,0}(X) \subset H^{1}(X ; \mathbb{C}$ ) (generated by the classes of closed holomorphic 
forms) and one has $H^{1,0} \cap$ conj $^{*} H^{1,0}=H^{1,0} \cap \bar{H}^{1,0}=0$ and either $b_{1}(X)=$ $2 h^{1,0}(X)$ or $b_{1}(X)=2 h^{1,0}(X)+1$. Thus, the worst that can happen is that the trace of conj* on $H^{1}(X ; \mathbb{R})$ is \pm 1 , and the first inequality should be replaced with $|\chi(\mathbb{R} X)-1| \leqslant \sigma^{-}(X)+1$. Alternatively, the Kähler property can be replaced, e.g., with the requirement $b_{1}(X)=0$. (Then the result holds for flexible varieties as well.) A stronger condition $\beta_{1}(X)=0$ would also imply the existence of $E$ as in the statement. Note also that the inequalities in 3.1.2 do not appeal to any covering; however, we do not know whether the statement still holds if there is no real double covering branched in $A$.

The ampleness condition in the last two inequalities can be replaced with $b_{1}(Y)=$ $b_{1}(X)$; otherwise $b_{1}(Y)-b_{1}(X)$ should be added to the right hand sides of both the inequalities. Note that, unlike 2.4.1, all the statements can be proved topologically following the lines of [A1]. (In order to prove the second inequality one should consider both the involutions $T_{ \pm}$on $Y$ and evaluate the inertia indices $\sigma_{ \pm}^{ \pm 1, \pm 1}$ of the restrictions of the intersection form to the bi-eigenspaces $H_{2}^{ \pm 1, \pm 1}$. The inequality would then follow from $\sigma_{-}^{ \pm 1, \mp 1} \geqslant 0$.) Moreover, taking into account various classes realized by the orientable components of $\mathbb{R} X$ and $\mathbb{R} Y$, one can obtain refined inequalities and their extremal properties. Here is an example:

3.1.3. Theorem. Let $X$ be a complex Kähler surface with real structure. Then

$$
-\sigma^{-}(X) \leqslant 1-\chi(\mathbb{R} X) \leqslant \sigma^{-}(X)-2 p_{+},
$$

where $p_{+}$is the number of orientable components of $\mathbb{R} X$ of positive Euler characteristic. If $A \subset X$ is a nonsingular ample even real divisor on $X$ so that there is a real divisor $E$ on $X$ with $2 E \sim A$, then

$$
\chi\left(\mathbb{R} X_{-}\right)-\chi\left(\mathbb{R} X_{+}\right) \leqslant \sigma^{-}(X)-b_{1}(X)+1+\frac{1}{4}[A]^{2}-\frac{1}{2} \chi(A)-2 q_{+},
$$

where $q_{+}$is the number of orientable components of $\mathbb{R} Y_{+}$of positive Euler characteristic (which, clearly, correspond to the components of $\mathbb{R} X_{+}$with positive Euler characteristic for which the restriction of the covering projection $\mathbb{R} Y_{+} \rightarrow \mathbb{R} X_{+}$is the orientation double covering.)

The extremal congruences 2.7.1, Arnol'd congruence 2.7.2, and Proposition 2.7.3 (as well as Lemma 2.7.6) are transferred to surfaces without changes. The generalized Arnol'd inequality 2.8.1 takes the following simpler form:

3.1.4. Arnol'd inequality for surfaces. Let $X$ be a Kähler surface with real structure. Then the number $p_{-}$of the orientable components of $\mathbb{R} X$ with negative Euler characteristic satisfies the inequality

$$
p_{-} \leqslant \frac{1}{2}\left(\sigma^{+}(X)-1\right),
$$

where $\sigma^{+}(X)=\frac{1}{2}\left[b_{2}(X)+\sigma(X)\right]$.

Below is another example of a refined statement specific for surfaces (see [Nik2]; in fact, one proves that the class $[\mathbb{R} X] \in H_{2}(X ; \mathbb{Z})$ is divisible by $\left.2^{r+1}\right)$. More examples of refined extremal congruences taking into account particular additional properties of the surface are found in 3.4 . 
3.1.5. Nikulin's congruence. Let $X$ be an $M$-surface with real structure and $H_{1}(X)=0$. Suppose that $\mathbb{R} X$ is orientable and that the Euler characteristic of each component of $\mathbb{R} X$ is divisible by $2^{r}$ for some $r \geqslant 1$. Then $\chi(\mathbb{R} X)=0 \bmod 2^{r+3}$.

If $X$ is a regular complete intersection in $\mathbb{P}^{q}$, then the complex ingredients of 3.1.1-3.1.4 (i.e., the topological invariants of $X$ and $A$ ) can easily be found by induction using 2.5.5.

3.1.6. Let a surface $X$ be a regular complete intersection in $\mathbb{P}^{q}$ of polydegree $\left(m_{1}, \ldots, m_{q-2}\right)$. Then

$$
\begin{gathered}
b_{1}(X)=0, \quad b_{2}(X)=\chi(X)-2, \\
\chi(X)=\mu_{q-2}\left(\mu_{1}^{2}-\mu_{2}-(q+1) \mu_{1}+\frac{1}{2} q(q+1)\right), \\
\sigma(X)=-\frac{1}{3} \mu_{q-2}\left(\mu_{1}^{2}-2 \mu_{2}-q-1\right),
\end{gathered}
$$

where $\mu_{i}$ is the $i$-th elementary symmetric polynomial in $\left(m_{1}, \ldots, m_{q-2}\right)$. If, besides, $A$ is a nonsingular curve cut on $X$ by a hypersurface of degree $m$, then

$$
\chi(A)=-m \mu_{q-2}\left(m+\mu_{1}-q-1\right) .
$$

3.2. The orbit space of the complex conjugation. Another phenomenon specific for surfaces is the fact that the fixed point set of the complex conjugation has codimension 2. Hence, the quotient $X /$ conj is a manifold; moreover, one can easily see that, up to isotopy, there is a unique smooth structure on $X /$ conj such that the projection $X \rightarrow X /$ conj is a double covering branched over $\mathbb{R} X$.

The resulting 4 -manifolds $X /$ conj form a very interesting class. On one hand, they are closely related to algebraic surfaces; on the other hand, in many respects their properties are just opposite to those of algebraic surfaces. S. Akbulut conjectured that the Seiberg-Witten invariants of the quotient vanish whenever the positive inertia index of the quotient is at least 2 . There is a strong evidence: if $\mathbb{R} X$ has a component of genus $\geqslant 2$, the vanishing of the invariants follows immediately from the 'smooth version' of the adjunction inequality applied to the image of the component in the quotient (for the appropriate version of the inequality, going back to Kronheimer and Mrowka, see [Sz]); if $\mathbb{R} X=\varnothing$, it was proved by Sh. Wang [Wa]. (If $\mathbb{R} X=\varnothing$, the projection $X \rightarrow X /$ conj is an honest double covering and one can easily control the behaviour of solutions to the Seiberg-Witten equation.) Furthermore, in many cases (see below) the quotient $X /$ conj is completely decomposable, i.e., splits into connected sum of copies of $\mathbb{P}^{2}, \overline{\mathbb{P}^{2}}$, and $S^{2} \times S^{2}$. (Recall that minimal algebraic surfaces are irreducible.) Thus, in many cases one can assert that $X /$ conj admits neither complex nor symplectic structure. There is a remarkable exception: if $X$ is a $K 3$-surface, $X /$ conj is diffeomorphic to either rational or Enriques surface, see below.

According to Arnol'd, the following result should be attributed to Maxwell:

3.2.1. Theorem. The orbit space $\mathbb{P}^{2} /$ conj is diffeomorphic to $S^{4}$.

The proof indicated below is found, e.g., in [Ma1]. It is a little bit shorter than the other, more direct, proofs published in $[\mathrm{Ku}]$, [Mas], [A3]. (Probably, except the one in [Mas]; as explained in [A4], this beautiful explicit proof was essentially 
known to Maxwell; a generalization of the statement to higher dimensions is also given in [A4]). The reason is the usage of the following Cerf theorem (see [C]): the group of diffeomorphisms of the 3-dimensional sphere coincides with the group of diffeomorphisms of the 4-dimensional ball (in short, $\Gamma_{4}=0$ ). The main advantage of this proof is that it can be generalized to other real surfaces, often using the Laundenbach-Poenaru theorem [LP], extending the Cerf theorem to handlebodies.

Proof. Pick a real flag $\mathbb{P}^{0} \subset \mathbb{P}^{1} \subset \mathbb{P}^{2}$. Its quotient by conj gives a simple cell decomposition of the quotient space: a 4-ball is attached to a 2-ball. The quotient of a closed round 4-ball centered at the origin of $\mathbb{P}^{2} \backslash \mathbb{P}^{1}$ is a 4 -ball, and the closure of its complement is a closed regular neighborhood of the 2 -ball $\mathbb{P}^{1} /$ conj. As is known, a closed regular neighborhood of a smooth 2-ball in a smooth 4-manifold is a smooth 4-ball. Thus, two 4-balls are patched together and, according to the Cerf theorem, the total space is diffeomorphic to $S^{4}$.

Most generalizations of 3.2.1 state that, under certain assumptions on the surface (usually, for surfaces explicitly constructed in a certain way so that one can control the topology of the quotient) the orbit space is completely decomposable. However, there are also a few examples of surfaces whose quotient is simply connected but not completely decomposable. Below we give a brief account of the known results.

For nonempty quadrics and cubics in $\mathbb{P}^{3}$ the decomposability result was obtain by M. Letizia [Let]. (Due to 3.2.1, a blow up at a real point does not change the orbit space; hence, for quadrics and connected cubics the result follows from 3.2.1.) For nonempty quartics and, more generally, nonempty $K 3$-surfaces the decomposability was proved by S. Donaldson [Don]. (Curiously, some $K 3$-surfaces, so called Fresnel-Kummer surfaces, are related to the Maxwell electro-magnetism theory.) Donaldson's approach is based on changing the complex structure in the twistor family and transforming the real structure to a holomorphic involution reversing the holomorphic forms; the quotient becomes a rational surface, which is obviously diffeomorphic to $\mathbb{P}^{2} \# k \overline{\mathbb{P}^{2}}$ or $S^{2} \times S^{2}$.

The complete decomposability of the quotient has been proved for double planes branched over curves of degree $2 k$ whose real part consists of a single nest of depth $k$ (S. Akbulut $[\mathrm{Ak}]$ ) or over curves obtained by small perturbation of union of real line (S. Finashin [F1]). Finashin extended these results to double quadrics branched over small perturbations of unions of generatrices and to certain regular complete intersections in $\mathbb{P}^{N}$ obtained recursively by perturbation of unions. For a wider class of plane curves he proved that the quotients of the corresponding double planes become completely decomposable after adding several copies of $\overline{\mathbb{P}^{2}}$.

The complete decomposability of the quotient holds for all real structures on rational surfaces (the proof is based on the classification of real rational surfaces, see 3.6.1) and for all real Enriques surfaces (using a modified version of Donaldson's trick, see the end of 3.7, Enriques surfaces can be reduced to rational ones). See [F2] for details.

An example of complex surface with real structure whose quotient is not completely decomposable is constructed by S. Finashin and E. Shustin [FS]. The quotient is a simply connected Spin-manifold whose signature is not 0 (and, hence, the manifold cannot be diffeomorphic to $k\left(S^{2} \times S^{2}\right)$ ). Currently it is not known whether there are surfaces whose quotient by complex conjugation do not decompose into connected sum of copies of $\mathbb{P}^{2}, \overline{\mathbb{P}^{2}}, S^{2} \times S^{2}$, and $K 3$-surface (the last option would allow Spin-manifolds with nonvanishing signature). 
3.3. Complex separation and Pontrjagin-Viro quadratic form. Let $X$ be a complex surface with real structure, $H_{1}(X)=0$, and $A$ a real curve on $X$. (We do not exclude the case $A=\varnothing$.) The pair $(X, A)$ is said to be of characteristic type if $[\mathbb{R} X]+[A]=w_{2}(X)$ in $H_{2}(X)$. Denote by pr: $X \rightarrow X^{\prime}$ the projection to the quotient space $X^{\prime}=X /$ conj. From the Smith exact sequence of the deck translation involution and the projection formula $w_{2}(X)=\operatorname{pr}^{*} w_{2}\left(X^{\prime}\right)+\mathrm{D}^{-1}[\mathbb{R} X]$ it follows that $\operatorname{rel} w_{2}\left(X^{\prime}\right)=\left[A^{\prime}\right]$ in $H_{2}\left(X^{\prime}, \mathbb{R} X\right)$ (where $A^{\prime}=A$ /conj). Hence, $[\mathbb{R} A]=\partial w_{2}\left(X^{\prime}\right)$ vanishes in $H_{1}(\mathbb{R} X)$ and there is a surface $\mathbb{R} X_{+}^{w} \subset \mathbb{R} X$ such that $\partial \mathbb{R} X_{+}^{w}=\mathbb{R} A$ and $\mathfrak{A}_{+}^{w}=\mathbb{R} X_{+}^{w} \cup A^{\prime}$ realizes $w_{2}\left(X^{\prime}\right)$. Let $\mathbb{R} X_{-}^{w}$ be the closure of $\mathbb{R} X \backslash \mathbb{R} X_{+}^{w}$. Since $\mathbb{R} X$ is homologous to zero in $X^{\prime}$, the surface $\mathfrak{A}_{-}^{w}=\mathbb{R} X_{-}^{w} \cup A^{\prime}$ also realizes $w_{2}\left(X^{\prime}\right)$. The decomposition $\mathbb{R} X=\mathbb{R} X_{+}^{w} \cup \mathbb{R} X_{-}^{w}$ satisfying these conditions is unique as, again due to the Smith exact sequence, $\operatorname{dim} \operatorname{Ker}\left[H_{2}(\mathbb{R} X) \rightarrow H_{2}\left(X^{\prime}\right)\right]=$ 1. It is called the complex separation of pair $(X, A)$.

Since the Arnol'd surfaces $\mathfrak{A}_{ \pm}^{w}$ are characteristic and $H_{1}(X)=0$, they possess canonical $\mathrm{Pin}^{-}$-structures and corresponding Rokhlin-Guillou-Marin forms, see C. Denote these forms by $\mathfrak{q}_{ \pm}^{w}$ and their restrictions to $\mathbb{R} X_{ \pm}^{w}$, by $q_{ \pm}^{w}$. It is easy to see that $q_{ \pm}^{w}$ vanishes on the kernel of the inclusion homomorphism $H_{1}\left(\mathbb{R} X_{ \pm}^{w}\right) \rightarrow H_{1}(\mathbb{R} X)$.

The following results are due to G. Mikhalkin [Mik1].

3.3.1. Lemma. Under the above assumptions one has

$$
\chi\left(\mathbb{R} X_{-}^{w}\right)=\frac{1}{4}[\chi(\mathbb{R} X)-\sigma(X)]+\frac{1}{2}[A]^{2}+\operatorname{Br} \mathfrak{q}_{-}^{w} \bmod 8 .
$$

3.3.2. Theorem. Let $X, A$ be as above. Assume that $q_{-}^{w}$ vanishes on the image of $H_{1}(\mathbb{R} A)$ and denote $R=\frac{1}{4}\left(\chi(\mathbb{R} X)-\sigma(X)+[A]^{2}\right)+\operatorname{Br} q_{-}^{w}$.

(1) If $A$ is an $M$-curve, then $\chi\left(\mathbb{R} X_{-}^{w}\right)=R \bmod 8$.

(2) If $A$ is an $(M-1)$-curve, then $\chi\left(\mathbb{R} X_{-}^{w}\right)=R \pm 1 \bmod 8$.

(3) If $A$ is an $(M-2)$-curve and $\chi\left(\mathbb{R} X_{-}^{w}\right)=R+4 \bmod 8$, then $A$ is of type $\mathrm{I}$.

(4) If $A$ is of type I, then $\chi\left(\mathbb{R} X_{-}^{w}\right)=R \bmod 4$.

Note that Lemma 3.3.1 and Theorem 3.3.2 are of topological nature and, hence, also hold for flexible curves on flexible surfaces; $A$ may even be nonorientable.

Proof of 3.3.1 and 3.3.2. Lemma 3.3.1 follows from the Rokhlin-Guillou-Marin congruence $[\mathrm{GM}]$ and a straightforward calculation, which gives $\left[\mathfrak{A}_{-}^{w}\right] \circ\left[\mathfrak{A}_{-}^{w}\right]=$ $\frac{1}{2}[A]^{2}-2 \chi\left(\mathbb{R} X_{-}^{w}\right)$. Proof of Theorem 3.3.2 repeats one of the proofs of the classical extremal congruences for plane curves (see [Ma2]); it is based on 3.3.1, additivity of the Brown invariant, and the following obvious facts: if $A$ is an $(M-d)$-curve, then $\operatorname{dim} H_{1}\left(A^{\prime}\right)=d$, and $A^{\prime}$ is orientable if and only if $A$ is of type I.

The following fact is an immediate consequence of the definition of the RokhlinGuillou-Marin form and 3.3.2:

3.3.3. Proposition. The restrictions of $\mathfrak{q}_{ \pm}^{w}$ to $H_{1}\left(A^{\prime}\right)$ differ by $2 \omega$, where $\omega$ is the characteristic class of the covering $A \backslash \mathbb{R} A \rightarrow A^{\prime} \backslash \mathbb{R} A$. As a consequence, $q_{ \pm}^{w}$ vanish on the image of $H_{1}(\mathbb{R} A)$ simultaneously, and in this case

$$
\operatorname{Br} q_{+}^{w}+\operatorname{Br} q_{-}^{w}=\frac{1}{2}\left(\chi(\mathbb{R} X)+\sigma(X)-[A]^{2}\right) \bmod 8
$$

and, if $A$ is of type $\mathrm{I}$,

$$
\chi\left(\mathbb{R} X_{+}^{w}\right)-\chi\left(\mathbb{R} X_{-}^{w}\right)=\operatorname{Br} q_{+}^{w}-\operatorname{Br} q_{-}^{w} \bmod 8 .
$$


(If flexible curves are admitted, in the first congruence $A$ must be orientable; the second one holds for nonorientable $A$ as well.)

The restrictions $q_{ \pm}^{w}$ can be calculated using Kalinin's spectral sequence (see A.2). We will show that $q_{ \pm}^{w}$ can be patched together into a quadratic form defined on $H_{1}(\mathbb{R} X)$, which depends only on the topology of $(X$, conj) and the class $[A] \in$ $\mathrm{H}_{2}(X ; \mathbb{Z})$.

Fix a class $c \in H_{2}(X ; \mathbb{Z})$ so that $\operatorname{conj}_{*} c=-c$ and $c=[\mathbb{R} X]+w_{2}(X) \bmod 2$. Pick an element $x \in H_{1}(\mathbb{R} X ; \mathbb{Z})$. There is an integral chain $x^{\prime}$ in $X$ such that $\partial x^{\prime}=x$. (One may have to multiply $x$ by an odd integer, which is irrelevant in what follows.) Then it is easy to see that $\left(1-\operatorname{conj}_{*}\right) x^{\prime}$ is an integral cycle and

$$
\mathcal{P}_{c}(x)=\left[\left(1-\operatorname{conj}_{*}\right) x^{\prime}\right]^{2}+c \circ\left(1-\operatorname{conj}_{*}\right) x^{\prime} \bmod 4
$$

does not depend on the choice of $x^{\prime}$. Furthermore, the function $x \mapsto \mathcal{P}_{c}(x)$ is a quadratic extension of the intersection form $(x, y) \mapsto x \circ y$ and, since $c$ dies in ${ }^{\infty} E_{2}$, one has $\mathcal{P}_{c}(-x)=\mathcal{P}_{c}(x)+2\left(c \circ \mathrm{bv}_{2} x\right)=\mathcal{P}_{c}(x)$, i.e., $\mathcal{P}_{c}$ factors through $H_{1}(\mathbb{R} X)$. The function $\mathcal{P}_{c}: H_{1}(\mathbb{R} X) \rightarrow \mathbb{Z}_{4}$ is called the Pontrjagin-Viro quadratic form.

3.3.4. Proposition. One has

(1) $\mathcal{P}_{c+2 e}(x)=\mathcal{P}_{c}(x)+2\left(e \circ \mathrm{bv}_{2} x\right)$ for any $e \in H_{2}(X ; \mathbb{Z}), \operatorname{conj}_{*} e=-e$;

(2) $\operatorname{Br} \mathcal{P}_{c}=\frac{1}{2}\left[\chi(\mathbb{R} X)+\sigma(X)-c^{2}\right] \bmod 8$;

(3) if $A$ is a (flexible) real curve on $X$ such that $(X, A)$ is of characteristic type, then $q_{ \pm}^{w}$ are the restrictions of $\mathcal{P}_{[A]}$ to $\mathbb{R} X_{ \pm}^{w}$.

Proof. (1) follows immediately from the definition; (3) is proved by direct calculation of indices of membranes. To prove (2) it suffices to construct an orientable flexible curve $A$ on $X$ such that the pair $(X, A)$ is of characteristic type and $\mathcal{P}_{[A]}$ vanishes on $\mathbb{R} A$ (e.g., $\mathbb{R} A=\varnothing$ ); the statement for $\mathcal{P}_{[A]}$ would follow then from (3) and 3.3.3, and it would extend to the other values of $c$ using (1) and B.1.5(3). Such a curve can easily be constructed: since $[\mathbb{R} X]+w_{2}(X)=0$ in ${ }^{\infty} E_{2}$, there is a class $f \in H_{2}(X ; \mathbb{Z})$ such that $\left(1-\operatorname{conj}_{*}\right) f=[\mathbb{R} X]+w_{2}(X) \bmod 2 H_{2}(X ; \mathbb{Z})$. Realize $f$ by a smooth orientable surface $F$ transversal to $\mathbb{R} A$ and perturb the singularities of $F \cup(-\operatorname{conj} F)$ according to the orientation.

Mention two special cases of the Pontrjagin-Viro form. An integral lift $c$ of $w_{2}(X)$ corresponds to a Spin ${ }^{\mathbb{C}}$-structure on $X$. Hence, if $[\mathbb{R} X]=0$ in $H_{2}(X)$, the correspondence $c \mapsto \mathcal{P}_{c}$ assigns to any $\operatorname{Spin}^{\mathbb{C}}$-structure on $X$ a Pin $^{-}$-structure on $\mathbb{R} X$ (cf. 2.9.2; the correspondence can be extended to all, not necessarily conj ${ }_{*}$-skewinvariant classes $c$ via 3.3.4(1)). There also is a similar correspondence in the case when $\mathbb{R} X$ is orientable, but not necessary homologous to zero; it is given by $c \mapsto \mathcal{P}_{c-[\mathbb{R} X]}$, where $[\mathbb{R} X]$ is the integral fundamental class of $\mathbb{R} X$ corresponding to some orientation. (In view of 3.3.4(1) his map does not depend on the orientation, as orientable components of $\mathbb{R} X$ annihilate $\mathrm{bv}_{2} H_{1}(\mathbb{R} X)$.)

The other special case worth mentioning is when $\mathbb{R} X$ itself is characteristic in $X$ (and, hence, $[A]=0 \bmod 2 H_{2}(X ; \mathbb{Z})$ ). In this case one can take $c=0$, and the resulting quadratic form $\mathcal{P}=\mathcal{P}_{0}$ is nothing but the Rokhlin-Guillou-Marin form on $\mathbb{R} X$. There is an alternative way to construct $\mathcal{P}$, which explains the name: it is easy to see that, since $[\mathbb{R} X]=w_{2}(X)$, the Pontrjagin square $P_{2}: H_{2}(X) \rightarrow \mathbb{Z}_{4}$ descends to ${ }^{2} E_{2}={ }^{\infty} E_{2}$, and one can let $\mathcal{P}=P_{2} \circ \mathrm{bv}_{2}: H_{1}(\mathbb{R} X) \rightarrow \mathbb{Z}_{4}$. In this form the construction admits generalizations to nonhomogeneous classes in $H_{*}(\mathbb{R} X)$ and 
to varieties of higher dimensions, not necessarily simply connected. Namely, let $X$ be a closed complex variety, $\operatorname{dim} X=2 n$, with real structure. (In fact, most statements can be modified to hold for an arbitrary closed manifold with orientation preserving involution whose dimension is divisible by 4 ; details can be found in [Deg3] or [DIK].) Assume that the Pontrjagin square $P_{2 n}: H_{2 n}(X) \rightarrow \mathbb{Z}_{4}$ descends to ${ }^{\infty} E_{2 n}(X)$ and define the Pontrjagin-Viro form via $\mathcal{P}=P_{2 n} \circ b_{2 n}: \mathcal{F}^{2 n} \rightarrow$ $\mathbb{Z}_{4}$. When defined, $\mathcal{P}$ is a quadratic extension of Kalinin's intersection pairing $*: \mathcal{F}^{2 n} \otimes \mathcal{F}^{2 n} \rightarrow \mathbb{Z}_{2}$.

The following statements are proved in [Deg3] (see also [DIK]).

3.3.5. Proposition. The Pontrjagin-Viro form is well defined if and only if the $W u$ class $u_{2 n}\left(X^{\prime} \backslash \mathbb{R} X\right)$ vanishes. In this case one has $\operatorname{Br} \mathcal{P}=\sigma(X) \bmod 8$.

3.3.6. Proposition. The Pontrjagin square $P_{2 n}$ descends to ${ }^{2} E_{2 n}$ if and only if $[\mathbb{R} X]$ realizes the $W u$ class $u_{2 n}(X)$. Thus, $[\mathbb{R} X]=u_{2 n}(X)$ is a necessary condition for $\mathcal{P}$ to be well defined. The following are sufficient conditions:

(1) $X$ is an $M$-variety;

(2) $[\mathbb{R} X]=u_{2 n}(X)$ and $X$ is $\mathbb{Z}_{2}$-Galois maximal (see A.1.4);

(3) $[\mathbb{R} X]=u_{2 n}(X)$ and $H_{i}(X)=0$ for $0<i<2 n$.

Assume now that $n=1$, i.e., $X$ is a surface. The condition $u_{2}\left(X^{\prime} \backslash \mathbb{R} X\right)=0$ in 3.3.5 is then equivalent to the requirement that $u_{2}\left(X^{\prime}\right)=w_{2}\left(X^{\prime}\right)$ belong to the image of the inclusion homomorphism $H_{2}(\mathbb{R} X) \rightarrow H_{2}\left(X^{\prime}\right)$, i.e., $\mathbb{R} X$ contains a subsurface characteristic in $X^{\prime}$. Such subsurfaces $F \subset \mathbb{R} X$ are characterized by the property $\mathcal{P}(x)=2([F] \circ x) \bmod 4$ for all $x \in \mathcal{F}^{2} \cap H_{0}(\mathbb{R} X)$. Given such a surface $F$, denote by $H_{F}$ the image of $\mathcal{F}^{2}$ under the natural map $\mathcal{F}^{2} \hookrightarrow H_{*}(\mathbb{R} X) \rightarrow$ $H_{1}(\mathbb{R} X) \rightarrow H_{1}(F)$ and define a quadratic function $\mathcal{P}_{F}: H_{F} \rightarrow \mathbb{Z}_{4}$ via

$$
x_{1} \mapsto \mathcal{P}\left(x_{1}+x_{0}\right)+2\left([F] \circ x_{0}\right),
$$

where $x_{0} \in H_{0}(\mathbb{R} X)$ is any element such that $x_{1}+x_{0} \in \mathcal{F}^{2}$.

3.3.7. Proposition. The function $\mathcal{P}_{F}: H_{F} \rightarrow \mathbb{Z}_{4}$ coincides with the RokhlinGuillou-Marin form of the characteristic surface $F \subset X^{\prime}$. In particular, $H_{F}$ coincides with the kernel of the inclusion homomorphism $H_{1}(F) \rightarrow H_{1}\left(X^{\prime}\right)$, the subspace $H_{F} \subset H_{1}(F)$ is informative (in respect to $\mathcal{P}_{F}$; see B.1 for the definition), and one has

$$
\chi(F)=\frac{1}{4}[\chi(\mathbb{R} X)-\sigma(X)]+\operatorname{Br} \mathcal{P}_{F} \bmod 8 .
$$

If, in addition, $H_{1}(X)=0$, the last statement implies 3.3.4(3) (for $A=\varnothing$ ); furthermore, in this case one can see that the complex separation defined in 3.3 can be expressed in terms of $\mathcal{P}$ : two components $C_{1}, C_{2}$ of $\mathbb{R} X$ belong to the same half $\mathbb{R} X_{+}^{w}$ or $\mathbb{R} X_{-}^{w}$ if and only if $\mathcal{P}\left(\left\langle C_{1}-C_{2}\right\rangle\right)=0$.

3.4. Spin-congruences. Below we reproduce two extremal congruences which take into account $w_{2}(X)$ and orientability of $\mathbb{R} X$. Other similar results, which employ more subtle information about the homotopy type of $X$, can be found in 3.7 (see also [DIK], devoted to detailed study of real Enriques surfaces; in fact, the congruences below are a by-product of the results and tools developed in [DIK]). 
3.4.1. Extremal congruence for non Spin-surfaces. Let $X$ be a complex closed surface with real structure and $w_{2}(X) \neq 0$.

(1) If $X$ is an $M$-surface, then $\mathbb{R} X$ is nonorientable.

(2) If $w_{2}(X)$ belongs to the image of Tors $H_{2}(X ; \mathbb{Z})$ in $H_{2}(X)$ and $\mathbb{R} X$ is orientable, then $X$ is an $(M-d)$-surface, $d \geqslant 2$, and

- if $d=2$, then $\chi(\mathbb{R} X)=\sigma(X) \bmod 16$;

- if $d=3$, then $\chi(\mathbb{R} X)=\sigma(X) \pm 2 \bmod 16$;

- if $d=4$ and $\chi(\mathbb{R} X)=\sigma(X)+8 \bmod 16$, then $\mathbb{R} X$ is of type $\mathrm{I}_{\text {tors }}$.

3.4.2. Extremal congruence for Spin-surfaces. Let $X$ be a complex closed surface with real structure and $w_{2}(X)=0$.

(1) If $H_{1}(X)=0$, then $\mathbb{R} X$ is orientable.

(2) If $b_{1}(X)=0$ and $\mathbb{R} X$ is nonorientable, then $X$ is an $(M-d)$-surface, $d \geqslant 2$, and

- if $d=2$, then $\chi(\mathbb{R} X)=\sigma(X) \bmod 16$;

- if $d=3$, then $\chi(\mathbb{R} X)=\sigma(X) \pm 2 \bmod 16$;

- if $d=4$ and $\chi(\mathbb{R} X)=\sigma(X)+8 \bmod 16$, then $\mathbb{R} X$ is of type $\mathrm{I}_{\text {tors }}$.

To prove the congruence part (Statement (2)) of 3.4.2 and 3.4.1 we show that a part of $T_{2}(X)=$ Tors $H_{2}(X ; \mathbb{Z}) \otimes \mathbb{Z}_{2} \subset H_{2}(X)$ dies in ${ }^{\infty} E_{2}$ : then $\operatorname{dim} J<d$ and the congruences follow (cf. 2.7).

Proof of 3.4.1. The class $w_{2}(X) \neq 0$ dies in ${ }^{\infty} E_{2}$ if and only if $\mathbb{R} X$ is orientable (see Table 1 in A.2.6), and the statement follows.

Proof of 3.4.2. (1) If there is $x_{1} \in H_{1}(\mathbb{R} X)$ with $x_{1}^{2}=1$, then $\left(\operatorname{bv}_{2} x_{1}\right)^{2}=1$. ( $\mathrm{bv}_{2} x_{1}$ is well defined since $H_{1}(X)=0$.) This contradicts to the assumption that the intersection form in $H_{2}(X)$ and, hence, in ${ }^{\infty} E_{2}$ is even.

(2) By the assumption, there is $x_{1} \in H_{1}(\mathbb{R} X ; \mathbb{Z})$ with $x_{1}^{2}=1$. Similar to (1) one concludes that $\mathrm{bv}_{1} x_{1} \notin \mathrm{bv}_{1} H_{0}(\mathbb{R} X)$. Furthermore, any nonorientable component $C_{i}$ of $\mathbb{R} X$ is of even genus (as otherwise $\left[C_{i}\right]^{2}=1 \bmod 2$ ). Then bv $x_{1} \notin$ $\mathrm{Sq}_{1}{ }^{\infty} E_{2}$ and, hence, $H_{2}(X) / H_{2}(X ; \mathbb{Z}) \otimes \mathbb{Z}_{2}$ is not covered by $\operatorname{Im} b_{2}$.

Remark. Alternatively, 3.4.2(1) can be derived from 2.9.1: if $H_{1}(X)=0$, the only Spin-structure on $X$ is obviously conj-invariant, and the construction of 2.9 .1 produces a canonical semi-orientation on $\mathbb{R} X$.

3.5. Surfaces in $\mathbb{P}^{3}$. In the next few section we cite the known classification results for surfaces. In order to describe the homeomorphism type of the real part of a surface we denote by $S_{p}, p \geqslant 0$, an orientable closed surface of genus $p$, $S_{p}=\#_{p}\left(S^{1} \times S^{1}\right)$, and by $V_{q}, q \geqslant 1$, a nonorientable closed surface of genus $q / 2$, $V_{q}=\#_{q} \mathbb{R P}^{2}$. (See 4.1 for our definition of genus in this case.) Let $S=S_{0}$.

From the topological point of view, surfaces in $\mathbb{P}^{3}$ are traditionally studied up to one of the following equivalence relations: homeomorphism of the real part of a surface, ambient isotopy of the real parts in $\mathbb{R P}^{3}$, rigid isotopy (i.e., isotopy in the class of nonsingular or, more generally, equisingular in some appropriate sense surfaces of the same degree), and rough projective equivalence (i.e., projective transformation and rigid isotopy). The difference between the last two relations is due to the fact that the group $P G L(4 ; \mathbb{R})$ of projective transformations of $\mathbb{R P}^{3}$ has 
two connected components. Certainly, the transformations in the component of unity transform a surface into a rigidly isotopic one.

For any degree the number of classes is finite (for each of the four equivalence relations); this follows from the fact that for each relation the universal family of hypersurfaces of a given degree admits a finite (semi-algebraic) stratification such that, in respect to the chosen relation, the family is locally trivial over each strata. (For topological equivalence relations the existence of a stratification was proved by A. Wallace [Wc] and A. N. Varchenko [Va1].)

Obviously, a real surface in $\mathbb{P}^{3}$ of degree 1 is a real projective plane. The classification of surfaces of degree 2 is a subject of classical analytic geometry. Up to rigid isotopy there are eight types of such surfaces (including singular ones), which are distinguished by the topology of their real parts. A nonsingular real surface $X \subset \mathbb{P}^{3}$ of degree 2 may be either hyperboloid $\left(\mathbb{R} X=S_{1}\right)$, or ellipsoid $(\mathbb{R} X=S)$, or empty surface $(\mathbb{R} X=\varnothing)$. Note that a nonsingular quadric $X$ is isomorphic to $\mathbb{P}^{1} \times \mathbb{P}^{1}$, and when considered as an abstract surface, $X$ admits four nonequivalent real structures: there are two real structures with empty real part.

In accordance with the general setting of this survey we consider nonsingular surfaces only. For such surfaces a complete classification (for each of the four equivalence relations) is known in degrees $\leqslant 4$. In degrees $\geqslant 5$ even the maximal number of connected components of the real part of a surface is not known. (Note that the maximum can always be realized by a nonsingular surface.) The best known upper bound is obtained by adding the Smith and Comessatti (PetrovskyOleinik) inequalities. It is worth mentioning that each inequality separately is sharp (see [V2], where the refined Comessatti inequality $1-\chi(\mathbb{R} X) \leqslant \sigma^{-}(X)-2 p_{+}$is also shown to be sharp). However, there is an evidence that the derived bounds for $\beta_{0}$ and $\beta_{1}$ are not sharp. Asymptotically one has $\max \beta_{i} \sim k_{i} d^{3}, i=0,1$, where $d$ is the degree, $\frac{13}{36} \leqslant k_{0} \leqslant \frac{5}{12}$, and $\frac{13}{18} \leqslant k_{1} \leqslant \frac{5}{6}$ (examples establishing the lower bounds are constructed by F. Bihan $[\mathrm{Bi}])$.

3.5.1. Real cubic surfaces. The classification of real surfaces in $\mathbb{P}^{3}$ of degree 3 is due to L. Schläfli [Sch], F. Klein [Kl], and H. G. Zeuthen [Ze].

3.5.2. There are five rigid isotopy types of nonsingular real cubic surfaces in $\mathbb{P}^{3}$. They are distinguished by their real parts, which are $V_{7}, V_{5}, V_{3}, V_{1} \sqcup S$, or $V_{1}$.

Proof. The topological invariants of the complex part of a nonsingular cubic surface $X \subset \mathbb{P}^{3}$ are given by 3.1.6: $\beta_{*}(X)=9, \sigma(X)=-4$, and $\sigma^{-}(X)=5$. Hence, the five homeomorphism types of the real part listed above are all not prohibited by the Smith inequality 3.1.1, extremal congruence 2.7.1, and Comessatti-Petrovsky inequality 3.1.2. Thus, it remains to show that all the five topological types are realized and that the space of nonsingular cubics has at most five connected components. Consider the space $\mathbb{R C}_{3}=\mathbb{R P}^{19}$ of all real cubic surfaces in $\mathbb{P}^{3}$ and let $\mathbb{R} \Delta \subset \mathbb{R C}$ be the set of singular cubics, $\Delta_{0} \subset \mathbb{R} \Delta$, the set of cubics with a single nondegenerate double point, and $\mathcal{S}=\mathbb{R} \Delta \backslash \Delta_{0}$. Then $\operatorname{dim} \mathbb{R} \Delta=18$ and $\operatorname{dim} \mathcal{S} \leqslant 17$, and from the Poincaré duality and the exact sequence of the triple $\left(\mathbb{R} \mathcal{C}_{3}, \mathbb{R} \Delta, \mathcal{S}\right)$ it follows that $\operatorname{dim} H^{0}\left(\mathbb{R C}_{3} \backslash \mathbb{R} \Delta\right)=1+\operatorname{dim} \operatorname{Ker}$ in $_{*}$, where $\operatorname{in}_{*}: H_{18}(\mathbb{R} \Delta, \mathcal{S}) \rightarrow H_{18}\left(\mathbb{R C}_{3}, \mathcal{S}\right)$ is induced by the inclusion. The group $H_{18}(\mathbb{R} \Delta, \mathcal{S})$ is generated by fundamental classes of the connected components of $\Delta_{0}$, i.e., rigid isotopy classes of cubics with a single nondegenerate double point. Such a cubic (assuming that the singular point is $(1: 0: 0: 0))$ is given by an equation of the 
form

$$
x_{0} p_{2}\left(x_{1}, x_{2}, x_{3}\right)+p_{3}\left(x_{1}, x_{2}, x_{3}\right)=0,
$$

where $p_{2}$ and $p_{3}$ are real homogeneous polynomials of degree 2 and 3 respectively. $(1: 0: 0: 0)$ is the only singular point of the surface and it is a nondegenerate double point if and only if the quadric $C_{2}=\left\{p_{2}=0\right\}$ is nonsingular and the cubic $C_{3}=$ $\left\{p_{3}=0\right\}$ intersects $C_{2}$ transversally. (Note that $C_{3}$ may be singular.) Through any set of six disjoint points of $C_{2}$ one can trace a cubic curve not containing $C_{2}$, and such curves form a 3 -dimensional affine space. Hence, $\Delta_{0}$ has at most five connected components: either $\mathbb{R} C_{2}=\varnothing$, or $\mathbb{R} C_{2} \cong S^{1}$ and there are $2 i, i=0,1,2,3$, real intersection points of $C_{2}$ and $C_{3}$. Perturbing the singular surfaces obtained in this way one can construct all the five topological types of nonsingular cubic surfaces.

It remains to prove that $\operatorname{dim} \operatorname{Kerin}_{*} \leqslant 4=\operatorname{dim} H_{18}(\mathbb{R} \Delta, \mathcal{S})-1$, and for this purpose, again due to the Poincaré duality, it suffices to construct a topological circle in $\mathbb{R} \mathcal{C}_{3} \backslash \mathcal{S}$ which intersects $\Delta_{0}$ transversally at two points in distinct strata. Such a circle is contained in the versal 2-parameter perturbation of the cuspidal surface given by

$$
x_{0}\left(x_{1}^{2}+x_{2}^{2}\right)+x_{3}^{3}+x_{2}^{3}=0 .
$$

Remark. Topologically, the $V_{q}$ component of the real part of a nonsingular cubic surface is situated in $\mathbb{R P}^{3}$ as the standard $\mathbb{R} \mathbb{P}^{2}$ with unlinked and unknotted handles attached. This can be deduced, e.g., from the construction of the surfaces indicated in the proof above.

3.5.3. Real quartic surfaces. The systematic study of the topology of the surfaces of degree 4 was launched by K. Rohn and D. Hilbert at the beginning of this century. (Hilbert posed this question as part of his 16-th problem.) However, first complete proofs of some of their statements appeared later and are due to I. Petrovsky and O. Oleinik (the bound on the number of spherical components) and to R. Thom (the bound on the total Betti number). The study was continued in the 60 th-70th by G. Utkin, and the classification was completed by $\mathrm{V}$. Kharlamov [Kh3], [Kh6] (the topological and isotopic classification; one isotopy class was eliminated in collaboration with V. Nikulin) and V. Nikulin [Nik1] (the rough projective classification). The chirality of degree 4 surfaces was studied by V. Kharlamov [Kh7], [Kh8].

As it is the case for any nonsingular real surface of even degree, the real part of a nonsingular quartic is orientable. Its position in $\mathbb{R P}^{3}$ is relatively simple: it is isotopic to a union of ellipsoids and hyperboloids with unknotted and unlinked handles. With one exception the components are outside each other; in the exceptional case the real part consists of two nested spheres. (In most cases these results can be derived from the Bezout theorem.)

With the only exception of two nested spheres the position of $\mathbb{R} X$ in $\mathbb{R P}^{3}$ is determined up to isotopy by the images of the inclusion homomorphisms $H_{1}\left(C_{j}\right) \rightarrow$ $H_{1}\left(\mathbb{R P}^{3}\right)=\mathbb{Z}_{2}$, where $C_{j} \subset \mathbb{R} X$ are the components of $\mathbb{R} X$. (This follows from general theorems of 3-dimensional topology and the fact that the handles are unknotted and unlinked.) For a nonspherical component we use the notation $S_{p}^{0}$ (respectively, $S_{p}^{1}$ ) if the image is (respectively, is not) trivial. Further, $n S$ stands for $n$ unnested spheres and $S(S)$, for a nest of two spheres.

In the case of degree 4 surfaces all the four classifications (topological, isotopic, rough projective, and rigid) are distinct. 


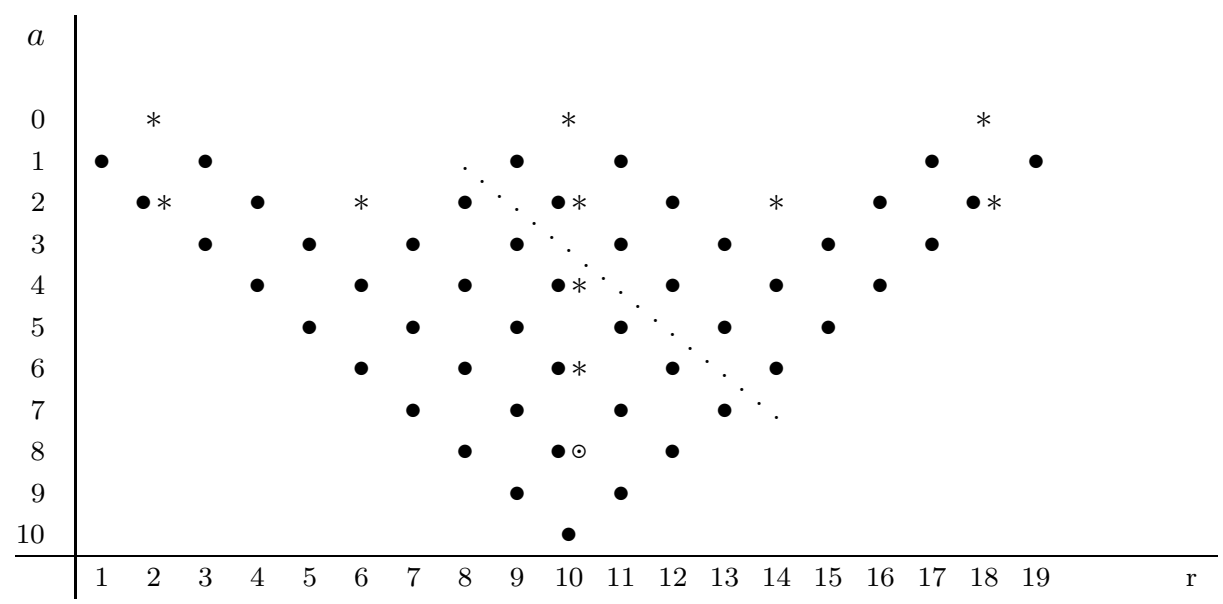

TABLE 1. Noncontractible quartic surfaces in $\mathbb{R}^{3}$

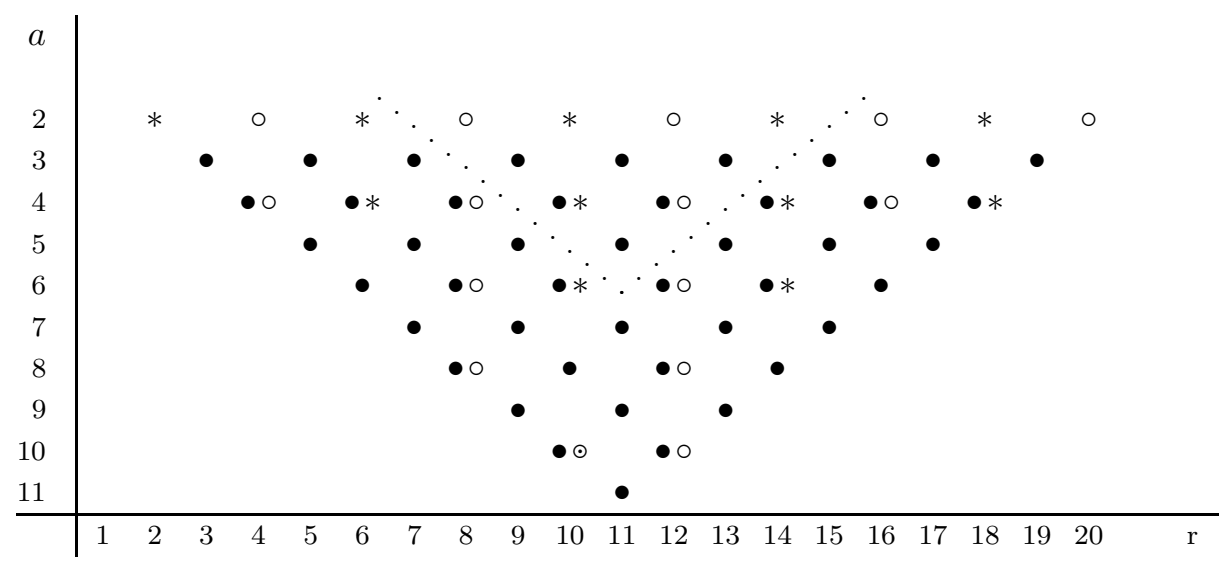

TABLE 2. Contractible quartic surfaces in $\mathbb{R P}^{3}$

* and $\odot$ stand for a surface of type $\mathrm{I}_{0}$, $\odot$ stands for a surface of type $\mathrm{I}_{\mathrm{hp}}$, and $\bullet$ stands for a surface of type II. The surfaces of type $\mathrm{I}_{0}$ denoted by $\odot$ have $\mathbb{R} X=2 S_{1}((a, r)=(8,10)$ in Table 1$)$ or $\mathbb{R} X=\varnothing((a, r)=(10,10)$ in Table 2$)$; the surfaces with $a+r=22$ (the right edge of Table 2) have $\mathbb{R} X=(r-10) S$; the other surfaces have $\mathbb{R} X=S_{g} \sqcup k S$, where $g=\frac{1}{2}(22-a-r)$ and $k=\frac{1}{2}(r-a)$. The surfaces below (respectively, above) the dotted lines are (respectively, are not) rigidly isotopic to their mirror images.

3.5.4. Classifications of real quartic surfaces. A real nonsingular surface of degree 4 is determined up to rigid isotopy by the isotopy type of its real part $\mathbb{R} X$, its complex type $\left(\mathrm{I}_{0}, \mathrm{I}_{\mathrm{hp}}\right.$, or II, see 2.6$)$ and its chirality, i.e., whether the surface is rigidly isotopic to its mirror image. The isotopy types of the real parts are those obtained by a sequence of Morse simplifications (see 3.7) from the following extremal types:

(1) $M$-surfaces: $S_{10}^{1} \sqcup S, S_{6}^{1} \sqcup 5 S, S_{2}^{1} \sqcup 9 S$;

(2) (M-2)-surfaces: $S_{7}^{1} \sqcup 2 S, S_{3}^{1} \sqcup 6 S, S_{a}^{0} \sqcup(9-a) S, 0 \leqslant a \leqslant 9$; 
(3) pairs of tori: $S_{1}^{1} \sqcup S_{1}^{1}, S_{1}^{0} \sqcup S_{1}^{0}$;

(4) pair of nested spheres: $S(S)$.

The following is the complete list of nonchiral isotopy types: $p S \sqcup S_{q}^{0}, p \geqslant 3, q \geqslant 3$, and $p S \sqcup S_{q}^{1}, p \geqslant 4$. The complex types are shown in Tables 1 and 2 .

Remark. As it follows from 3.5.4, with the exception of the case $\mathbb{R} X=S(S)$ the isotopy type of the real part $\mathbb{R} X$ of a real quartic surface in $\mathbb{P}^{3}$ is determined by its topological type and whether $\mathbb{R} X$ is contractible in $\mathbb{R} \mathbb{P}^{3}$ or not.

3.5.5. Surfaces of degree 5. Starting from degree 5, one observes the following phenomenon: on one hand, any surface obtained by a small deformation of a surface of a given degree in $\mathbb{P}^{3}$ can be embedded in $\mathbb{P}^{3}$ (as a surface of the same degree); on the other hand, by a big deformation one can obtain a surface which can not be embedded in $\mathbb{P}^{3}$. (It is worth mentioning that in degree 4 the situation is just the opposite: a nonsingular quartic surface can be made nonprojective by a small deformation, and, on the other hand, any surface of the same deformation class, i.e., a $K 3$-surface, see 3.7.2 below, can be made a nonsingular quartic by a small deformation. This explains why the topological classification of real nonsingular degree 4 surfaces is the same as the topological classification of real $K 3$-surfaces.)

In degree 5 the phenomenon was studied by E. Horikawa [Ho]. He proved that the base of a versal deformation of a nonsingular quintic consists of two smooth irreducible components $M_{0}, M_{1}$ intersecting normally, $\operatorname{dim}_{\mathbb{C}} M_{0}=\operatorname{dim}_{\mathbb{C}} M_{1}=40$, and $\operatorname{dim}_{\mathbb{C}} M_{0} \cap M_{1}=39$. The points of $M_{0} \backslash\left(M_{0} \cap M_{1}\right)$ correspond to nonsingular quintics, those of $M_{1} \backslash\left(M_{0} \cap M_{1}\right)$, to double coverings of $\mathbb{P}^{1} \times \mathbb{P}^{1}$, and those of $M_{0} \cap M_{1}$, to double coverings of the quadratic cone $\Sigma_{2}$. Certainly, the deformation of a real quintic can be made equivariant and its members can be used to produce quintics of the same real deformation class. This observation was used to prove the existence part of 3.5.6 and 3.5.7.

3.5.6. Proposition. The extremal values of the Betti numbers of nonsingular quintics in $\mathbb{P}^{3}$ are in the range $22 \leqslant \max \beta_{0} \leqslant 25, \max \beta_{1}=45$ or 47 . For the surfaces in the same deformation class one has $23 \leqslant \max \beta_{0} \leqslant 25$ and $\max \beta_{1}=47$.

Proof. For a nonsingular quintic $X$ one has $h^{1,1}(X)=45, \beta_{*}(X)=55$, and $\sigma(X)=-35$. Thus, the upper bounds follow from the Smith and Comessatti inequalities 3.1.1, 3.1.2 and the extremal congruence 2.7.1. Quintics with $\beta_{1}=45$ were constructed by V. Kharlamov [Kh9], quintics with $\beta_{0}=22$, by I. Itenberg and V. Kharlamov [IKh], and $\mathbb{C}$-deformation equivalent surfaces with $\beta_{0}=23$ and those with $\beta_{1}=47$, by F. Bihan [Bi].

3.5.7. Proposition. The real part of an $M$-surface $\mathbb{C}$-deformation equivalent to a nonsingular quintic can only have $5,9,13,17,21$, or 25 connected components. $M$-quintics with 5, 9, 13, 17, and 21 connected components of the real part do exist.

Proof. The other values are prohibited by the extremal congruence 2.7.1 and the Smith inequality 3.1.1. $M$-surfaces with $5,9,13,17$, and 21 components were constructed by V. Kharlamov [Kh9] (the construction for 17 and 21 components is based on some auxiliary curves constructed by O. Viro).

The $M$-surfaces constructed in [Kh9] are

$$
20 S \sqcup V_{13}, \quad 16 S \sqcup V_{21}, \quad 12 S \sqcup V_{29}, \quad 8 S \sqcup V_{37}, \quad 4 S \sqcup V_{45} .
$$


Their twins, obtained by switching the sign of the double covering, are

$$
4 S \sqcup V_{43}, \quad 8 S \sqcup V_{35}, \quad 12 S \sqcup V_{27}, \quad 11 S \sqcup S_{1} \sqcup V_{25}, \quad 16 S \sqcup V_{19} .
$$

Among other pairs constructed at the moment are $19 S \sqcup S_{2} \sqcup V_{7}, 4 S \sqcup V_{43}$ and $19 S \sqcup S_{1} \sqcup V_{7}, 4 S \sqcup V_{45}$. As it follows from the construction of the auxiliary curves, the Morse simplifications of all the topological types above can be realized by real quintics. The variety of the examples obtained in this way has never been analyzed systematically.

The surface with 22 components constructed by V. Kharlamov and I. Itenberg in [IKh] is $21 S \sqcup V_{7}$. Its twin is $S \sqcup S_{2} \sqcup V_{41}$, with $\beta_{1}=45$.

Recently B. Chevallier (unpublished) found some additional examples. His approach looks different (it is based on Viro's patching method), but there is an evidence that both the approaches are close or even equivalent: the approach based on the Horikawa theorem also relies on Viro's method to construct auxiliary curves, while Chevallier's approach also uses double quadrics. The new types with the total Betti number or Euler characteristic close to extremal ones are

$$
\begin{gathered}
18 S \sqcup S_{1} \sqcup V_{9}, \quad 17 S \sqcup 2 S_{1} \sqcup V_{3}, \quad 17 S \sqcup S_{2} \sqcup V_{5}, \ldots, 4 S \sqcup S_{8} \sqcup V_{23}, \\
3 S \sqcup S_{1} \sqcup V_{39}, \quad 3 S \sqcup S_{7} \sqcup V_{27}, \quad 2 S \sqcup S_{1} \sqcup V_{41}, \quad S \sqcup S_{18} \sqcup V_{3}, \quad S \sqcup S_{10} \sqcup V_{21} .
\end{gathered}
$$

It is worth mentioning that if an $M$-quintic with 25 components does exist, then, according to the known inequalities and congruences, it must be of one of the following topological types:

$$
24 S \sqcup V_{5}, \quad 23 S \sqcup S_{1} \sqcup V_{3}, \quad 23 S \sqcup S_{2} \sqcup V_{1}, \quad 22 S \sqcup S_{1} \sqcup S_{1} \sqcup V_{1} .
$$

The two last types are prohibited (the forth one, O. Viro, and the third one, by V. Kharlamov and I. Itenberg, cf.[Kh10]). The problem of existence of $M$-surfaces of degree 5 of the two other topological types is still open.

3.6. Rational Surfaces. In accordance with the general setting of this survey, by a real rational surface we mean a rational (over $\mathbb{C}$ ) surface supplied with a real structure. It is worth emphasizing that such a surface does not need to be rational over $\mathbb{R}$; Theorem 3.6.1 below gives plenty of examples. Real rational surfaces were classified by Comessatti [Co2]-[Co4]. Following Comessatti's approach, let us confine ourselves to minimal (over $\mathbb{R}$ ) real rational surfaces. (Recall that a nonsingular surface $X$ is called (relatively) minimal if any holomorphic degree 1 map $X \rightarrow X^{\prime}$ is a biholomorphism. A real surface $(X$, conj) is minimal over $\mathbb{R}$ if the above condition holds for conj-equivariant maps. A surface is minimal over $\mathbb{C}$ if and only if it does not contain a (-1)-curve, and it is minimal over $\mathbb{R}$ if and only if it does not contain a real (-1)-curve or a pair of disjoint conjugate $(-1)$-curves.)

3.6.1. Minimal real rational surfaces. The following is a complete list of rigid isotopy types of minimal over $\mathbb{R}$ rational surfaces with anti-holomorphic involution:

(1) real projective plane $\mathbb{P}^{2}: \mathbb{R} X=V_{1}$;

(2) real quadric $\mathbb{P}^{1} \times \mathbb{P}^{1}$ : there are four types: $\mathbb{R} X=S_{0}, \mathbb{R} X=S_{1}$, and two nonequivalent surfaces with $\mathbb{R} X=\varnothing$;

(3) ruled rational surfaces $\Sigma_{m}, m \geqslant 2$ : 
$m$ even: $\mathbb{R} X=\varnothing$ or $S_{1}, \quad \quad m$ odd: $\quad \mathbb{R} X=V_{2} ;$

(4) real conic bundles over $\mathbb{P}^{1}$ whose reducible fibers are all real and consist of pairs of complex conjugate exceptional curves: $\mathbb{R} X=m S$, where $2 m \geqslant 4$ is the number of reducible fibers;

(5) Del Pezzo surfaces of degree $d=K^{2}=1$ or 2 :

$$
d=1: \quad \mathbb{R} X=V_{1} \sqcup 4 S, \quad d=2: \quad \mathbb{R} X=3 S \text { or } 4 S .
$$

(From the complex point of view a Del Pezzo surface of degree $d \leqslant 9$ is obtained from $\mathbb{P}^{2}$ by blowing up $(9-d)$ points in general position, i.e., such that no three of them lie in a line and no six of them lie in a quadric.) Note that the Del Pezzo surface of degree 2 with $\mathbb{R} X=3 S$ can also be represented as a conic bundle over $\mathbb{P}^{1}$ with six reducible fibers. Note also that in the above list only surfaces (1)-(3) are minimal over $\mathbb{C}$.

Theorem 3.6.1 depends on a classification of anti-birational transformations of $\mathbb{P}^{2}$, which, in turn, is based on considering the fundamental points of such a transformation. A representation of Comessatti's proof in the modern language can be found in $[\mathrm{CP}]$.

3.7. Abelian, K3-, and Enriques Surfaces. The next group in the classification of complex algebraic surfaces are surfaces of Kodaira dimension 0, i.e., those whose minimal models are either abelian, or hyperelliptic, or $K 3$-, or Enriques surfaces, each type, except hyperelliptic surfaces, forming one deformation family. Since the minimal model of a complex surface of Kodaira dimension $\geqslant 0$ is unique (see, e.g., Hartshorne [Ha]), it must be real if the original surface is real. Hence, it suffices to consider surfaces minimal over $\mathbb{C}$; any other surface can be obtained from a minimal (over $\mathbb{C}$ ) one by a sequence of blow-ups at real points or pairs of complex conjugate points.

The classification of real hyperelliptic surfaces is still in progress. Below we treat the other three types.

An abelian surface $X$ (which topologically is a 4 -torus $S^{1} \times S^{1} \times S^{1} \times S^{1}$ ) can be characterized by $b_{1}(X)=4$ and $K_{X}=0$. Anti-holomorphic involutions on such surfaces were classified by Comessatti [Co4], whose result is the following:

3.7.1. Real Abelian Surfaces. Up to equivariant deformation an anti-holomorphic involution conj on a complex torus $X$ is determined by the real point set $\mathbb{R} X$ and, if $\mathbb{R} X=\varnothing$, by the action of $\operatorname{conj}_{*}$ on $H_{1}(X ; \mathbb{Z})$. There are five classes: $\mathbb{R} X=k S_{1}$ with $k=1,2,4$ and two classes with $\mathbb{R} X=\varnothing$.

A description of the moduli space of algebraic abelian surfaces with nonempty real part can be found in Silhol [Sil].

Sketch of the proof. Consider the uniformization $X=\mathbb{C}^{2} / L$, where $L \subset \mathbb{C}^{2}$ is a lattice of rank 4, and lift conj to an anti-automorphism $c$ of $\mathbb{C}^{2}$. If $\mathbb{R} X \neq \varnothing$, the lift can be chosen to be an involution preserving $L$. Since $c$ has eigenvalues \pm 1 of multiplicity 2 each, there are three isomorphism classes of the restriction $\left.c\right|_{L}$, and for each class there is a unique up to homotopy equivariant embedding to $\mathbb{C}^{2}$ (considered with the standard conjugation).

If $\mathbb{R} X=\varnothing$, the above arguments apply to the adjoint action Ad conj on $X$ considered as group, and conj itself is the composition of Ad conj and the affine shift by an element $a \in \operatorname{Ker}(1+\mathrm{Ad}$ conj $)$, which is to be considered up to $\operatorname{Im}(1-\mathrm{Ad}$ conj). Up to isomorphism, there are two solutions, one with Ad conj = id, and one with 


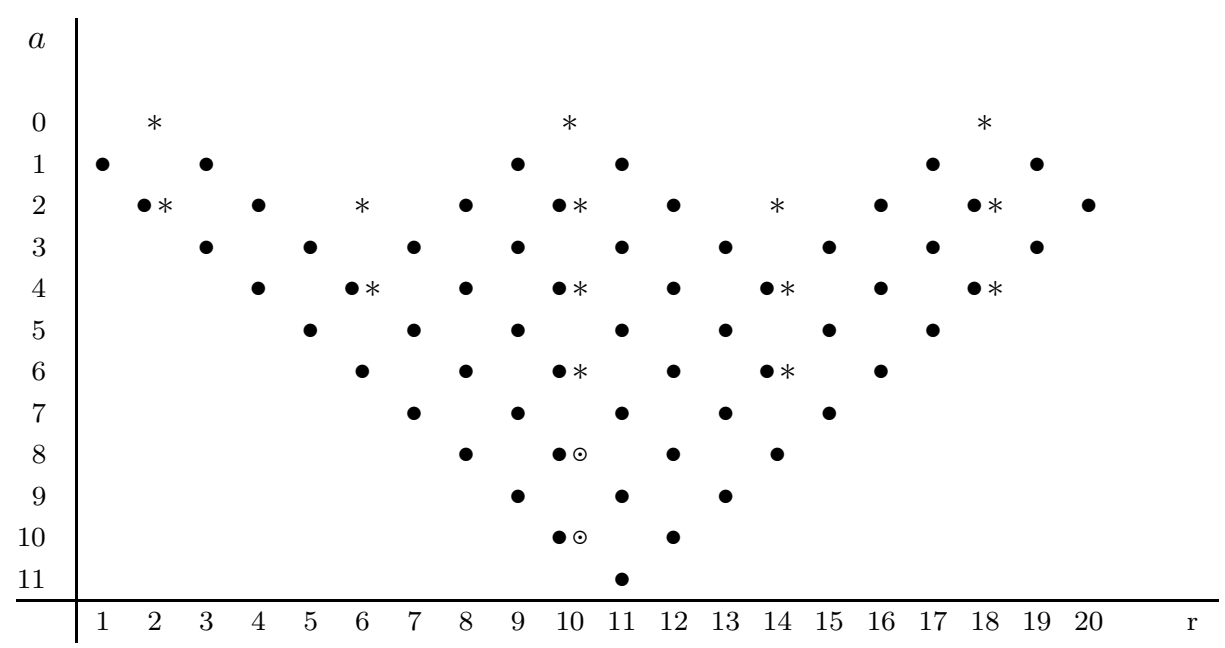

TABLE 3. Topological types of real $K 3$-surfaces

* and $\odot$ stand for a surface of type I and $\bullet$ stands for a surface of type II. The surfaces of type I denoted by $\odot$ have $\mathbb{R} X=2 S_{1}((a, r)=(8,10))$ or $\mathbb{R} X=\varnothing((a, r)=(10,10))$; the surfaces with $a+r=22$ (the right edge) have $\mathbb{R} X=(r-10) S$; the other surfaces have $\mathbb{R} X=S_{g} \sqcup k S$, where $g=\frac{1}{2}(22-a-r)$ and $k=\frac{1}{2}(r-a)$.

Ad conj $=\operatorname{id} \oplus \sigma$, where $\sigma$ is a real structure on an elliptic curve $C^{\prime}=\mathbb{C} / L^{\prime}$ such that $\mathbb{R} C=S^{1}$.

A complex $K 3$-surface $X$ is characterized by $b_{1}(X)=0, K_{X}=0$. (Topologically $X$ is a simply connected 4 -manifold with $\left.H_{2}(X ; \mathbb{Z})=2\left(-E_{8}\right) \oplus 3 U\right)$. In order to state the classification result for real $K 3$-surfaces, let us introduce the following notion: a topological type (i.e., a class of surfaces with homeomorphic real parts) is called extremal (within a fixed deformation family of complex surfaces) if it cannot be obtained from another topological type by a Morse simplification, i.e., Morse modofication decreasing the total Betti number. (A Morse simplification is either removing a spherical component or contracting a handle.) The following result is due to V. Kharlamov [Kh3] and N. Nikulin [Nik1]:

3.7.2. Real $K 3$-surfaces. A $K 3$-surface $X$ with anti-holomorphic involution is determined up to equivariant deformation by the topological type of its real part $\mathbb{R} X$ and its type (i.e., whether $[\mathbb{R} X]$ is or is not homologous to zero in $H_{2}(X)$, see 2.6). The topological types of the real parts are those and only those which can be obtained by a sequence of Morse simplifications from the following extremal types:

(1) $M$-surfaces: $S_{10} \sqcup S, S_{6} \sqcup 5 S, S_{2} \sqcup 9 S$;

(2) $(M-2)$-surfaces: $S_{7} \sqcup 2 S, S_{3} \sqcup 6 S$;

(3) pair of tori: $2 S_{1}$.

The topological types of the real part of a real K3-surface are listed in Table 3.

Proof of Theorem 3.7.2 is based on the global Torelli theorem for $K 3$-surfaces, see $[\mathrm{BPV}]$, which describes their moduli space. This reduces the problem of classification of anti-holomorphic involutions on $K 3$-surfaces up to rigid isotopy to the arithmetical problem of classification of involutive isometries on $2\left(-E_{8}\right) \oplus 3 U$ up to automorphism of the lattice. We omit the details; see [Nik1] or [DIK]. 
The remaining deformation type, Enriques surfaces, can be characterized by $2 K_{X}=0, K_{X} \neq 0$. (Another definition is the following: $X$ is an Enriques surface if $\pi_{1}(X)=\mathbb{Z}_{2}$ and the universal covering of $X$ is a $K 3$-surface.) Like the previous cases, a real Enriques surface $X$ is determined up to deformation by the topology of the complex conjugation involution conj: $X \rightarrow X$. However, the topology of the real part $\mathbb{R} X$ and its type are not sufficient anymore to determine the topology of the involution; one needs more sophisticated invariants. Below we cite the principal results on the deformation classification of real Enriques surfaces; details and a complete list of the deformation classes are found in [DIK].

3.7.3. Topology of the real part. There are 87 topological types of Enriques surfaces with anti-holomorphic involution. Each of them can be obtained by a sequence of Morse simplifications from one of the 22 extremal types listed below. Conversely, with the exception of the two types $6 S_{0}$ and $S_{1} \sqcup 5 S_{0}$, any topological type obtained in this way is realized by a real Enriques surface.

The 22 extremal types are:

(1) $M$-surfaces:

$$
\begin{array}{clll}
\text { (a) } \chi(\mathbb{R} X)=8: & & (\text { b) } \chi(\mathbb{R} X)=-8: & \\
4 V_{1} \sqcup 2 S_{0}, & 2 V_{2} \sqcup 4 S_{0}, & V_{11} \sqcup V_{1}, & V_{8} \sqcup V_{4}, \\
V_{2} \sqcup 2 V_{1} \sqcup 3 S_{0}, & V_{4} \sqcup 5 S_{0}, & V_{10} \sqcup V_{2}, & V_{7} \sqcup V_{5}, \\
V_{3} \sqcup V_{1} \sqcup 4 S_{0}, & V_{2} \sqcup S_{1} \sqcup 4 S_{0}, & V_{9} \sqcup V_{3}, & 2 V_{6}, \\
& & \\
& & \\
\text { (2) }(M-2) \text {-surfaces with } \chi(\mathbb{R} X)=0: & & \\
V_{4} \sqcup 2 V_{1}, & V_{5} \sqcup V_{1} \sqcup S_{0}, & 2 V_{3} \sqcup S_{0}, & \\
V_{3} \sqcup V_{2} \sqcup V_{1}, & V_{4} \sqcup V_{2} \sqcup S_{0}, & V_{4} \sqcup S_{1} \sqcup S_{0}, & 2 V_{2} \sqcup S_{1} ;
\end{array}
$$

(3) pair of tori: $2 S_{1}$.

Let $X$ be a real Enriques surface and $\tilde{X} \rightarrow X$ the covering $K 3$-surface. The real structure conj on $X$ lifts to two commuting real structures $t^{(1)}, t^{(2)} \tilde{X} \rightarrow \tilde{X}$, whose real parts $\mathbb{R} \tilde{X}^{(1)}, \mathbb{R} \tilde{X}^{(2)}$ are disjoint. The images $\mathbb{R} X^{(i)}$ of $\mathbb{R} \tilde{X}^{(i)}, i=1,2$, are also disjoint. Thus, the real part $\mathbb{R} X$ of $X$ splits into two subsets $\mathbb{R} X^{(1)}, \mathbb{R} X^{(2)}$ (which are unions of whole components), called halves. The half decomposition $\mathbb{R} X=\mathbb{R} X^{(1)} \cup \mathbb{R} X^{(2)}$ is an important topological (and deformation) invariant of the surface; it is designated by an expression of the form

$$
\mathbb{R} X=\left\{\text { components of } \mathbb{R} X^{(1)}\right\} \sqcup\left\{\text { components of } \mathbb{R} X^{(2)}\right\} .
$$

Clearly, one can speak about the type $\left(\mathrm{I}_{0}, \mathrm{I}_{u}\right.$, or II) of each half $\mathbb{R} X^{(i)}$ separately, as well as about the types (I or II) of $\mathbb{R} \tilde{X}^{(i)}$. Furthermore, $\mathbb{R} X^{(1)}$ is naturally identified with the fixed point set of the descent of $t^{(1)}$ to $\tilde{X} / t^{(2)}$; thus, one can also consider the type of $\mathbb{R} X^{(1)}$ in $\tilde{X} / t^{(2)}$. The latter quotient is diffeomorphic to a rational surface, if $\mathbb{R} X^{(2)} \neq \varnothing$, or to an Enriques surface otherwise. In the former case the possible types are I and II, in the latter case, $\mathrm{I}_{0}, \mathrm{I}_{u}$, and II.

3.7.4. Proposition. The covering $\mathbb{R} \tilde{X}^{(1)} \cup \mathbb{R} \tilde{X}^{(2)} \rightarrow \mathbb{R} X$ is the orientation double covering.

3.7.5. Half decomposition. Each half of a real Enriques surface is either

(1) $\alpha V_{g} \sqcup a V_{1} \sqcup b S$ with $g>1, a \geqslant 0, b \geqslant 0, \alpha=0,1$, or

(2) $2 V_{2}$, or

(3) $S_{1}$. 


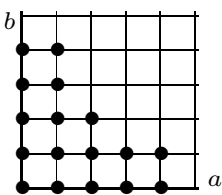

$\left\{a S_{0}\right\} \sqcup\left\{b S_{0}\right\}$, $\left\{V_{2} \sqcup a S_{0}\right\} \sqcup\left\{b S_{0}\right\}$

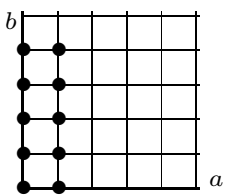

$\left\{V_{4} \sqcup a S_{0}\right\} \sqcup\left\{b S_{0}\right\}$

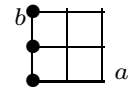

$\left\{V_{6} \sqcup a S_{0}\right\} \sqcup\left\{b S_{0}\right\}$

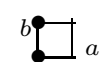

$\left\{V_{8} \sqcup a S_{0}\right\} \sqcup\left\{b S_{0}\right\}$, $\left\{V_{10} \sqcup a S_{0}\right\} \sqcup\left\{b S_{0}\right\}$

Figure 4. Half decompositions of exceptional real Enriques surfaces

With the exception of the cases $\mathbb{R} X=k S$ and $\mathbb{R} X=V_{2 r} \sqcup k S$ a half decomposition of a real part $\mathbb{R} X$ as in 3.7 .3 is realizable if and only if it satisfies (1)-(3) above. The realizable half decompositions of the exceptional real parts are listed in Figure 4.

Proposition 3.7.4 follows essentially from the fact that $\tilde{X}$ is Spin and $X$ is not; thus, the Enriques involution reverses the Spin-orientation of $\mathbb{R} \tilde{X}$ (see 2.9.1). Together with the restrictions to the topology of $\mathbb{R} \tilde{X}$ (see 3.7.2) this gives the possible forms (1)-(3) for a half of $\mathbb{R} X$. The exceptional half decompositions are treated using certain congruences similar to the extremal congruences 2.7.1.

In order to prohibit almost all nonexisting topological types it suffices to combine 3.7.4 (and 3.7.2) with the Smith inequality 3.1.1, generalized ComessattiPetrovsky inequality 3.1.2, and extremal congruences 2.7.1. After that, there are only two types, $S_{1} \sqcup 5 S_{0}$ and $3 V_{2}$, and one series, $S_{1} \sqcup V_{1} \sqcup \ldots$, still left to be prohibited. This is done by considering Kalinin's spectral sequence (see [DIK] for details).

For a technical reason real Enriques surfaces are subdivided into three classes, those of hyperbolic, parabolic, or elliptic type, depending on whether the minimal Euler characteristic of the components of $\mathbb{R} X$ is negative, zero, or positive.

3.7.6. Deformation classes. With few exceptions listed below the deformation class of a real Enriques surface $X$ with a distinguished half $\mathbb{R} X^{(1)}$ is determined by the topology of its half decomposition. The exceptions are:

(1) $M$-surfaces of parabolic and elliptic type, i.e., those with $\mathbb{R} X=2 V_{2} \sqcup 4 S$, $V_{2} \sqcup 2 V_{1} \sqcup 3 S$, or $4 V_{1} \sqcup 2 S$; the invariant is the Pontrjagin-Viro form;

(2) surfaces with $\mathbb{R} X=2 V_{1} \sqcup 4 S$;

(3) other surfaces with a half $\mathbb{R} X^{(1)}=4 S$; the additional invariants are the types, $\mathrm{I}_{u}, \mathrm{I}_{0}$, or II, of $\mathbb{R} X^{(1)}$ in $X$ and $\tilde{X} / t^{(2)}$;

(4) surfaces with $\mathbb{R} X=\left\{V_{10}\right\} \sqcup\{\varnothing\},\left\{V_{4} \sqcup S\right\} \sqcup\{\varnothing\},\left\{V_{2} \sqcup 4 S\right\} \sqcup\{\varnothing\}$, and $\{2 S\} \sqcup\{2 S\}$; the additional invariant is the type, $\mathrm{I}_{u}$ or $\mathrm{I}_{0}$, of $\mathbb{R} X$ in $X$;

(5) surfaces with $\mathbb{R} X=2 V_{1} \sqcup 3 S$; the additional invariant is the type, $\mathrm{I}_{u}$ or II, of $\mathbb{R} X$ in $X$;

(6) surfaces with $\mathbb{R} X=\left\{S_{1}\right\} \sqcup\left\{S_{1}\right\}$.

The additional invariant needed to distinguish the deformation classes in cases 3.7.6(2) and (6) are more sophisticated and depend on the special arithmetical properties of the induced $\left(\mathbb{Z}_{2} \times \mathbb{Z}_{2}\right)$-action on $H_{2}(\tilde{X} ; \mathbb{Z})$ (in case $(2)$ ) or the topology of mutual position of the two halves $\mathbb{R} \tilde{X}^{(i)}=S_{1} \sqcup S_{1}$ in $\tilde{X}$ (in case (6)).

The deformation classification of real Enriques surfaces is based upon two different approaches: Donaldson's trick and arithmetical calculations using the global 
Torelli theorem for $K 3$-surfaces. (Although each of the two approaches could do the job, the complexity of the resulting auxiliary problem depends essentially on the surface in question. The former, more geometric approach, works well for surfaces of hyperbolic and parabolic types; the latter, for those of elliptic type.) Donaldson's trick employs the hyper-Kähler structure on $\tilde{X}$ : one can change the complex structure so that the Enriques involution becomes anti-holomorphic and $t^{(2)}$ becomes holomorphic; then the quotient $\tilde{X} / t^{(2)}$ is a real rational surface (unless $\mathbb{R} X^{(2)}=\varnothing$ ) and the problem reduces to the study of real rational surfaces with a nonsingular anti-bicanonical curve (the image of $\mathbb{R} X^{(2)}$ ). In the second approach one uses the global Torelli theorem to establish a one-to-one correspondence between the deformation classes of real Enriques surfaces and the isomorphism classes of $\left(\mathbb{Z}_{2} \times \mathbb{Z}_{2}\right)$ actions (induced by $t^{(1)}$ and $\left.t^{(2)}\right)$ on the intersection lattice $H_{2}(\tilde{X} ; \mathbb{Z})=2\left(-E_{8}\right) \oplus 3 U$; the action must satisfy certain arithmetic conditions to ensure the realizability by anti-holomorphic involutions and the freeness of the composition $t^{(1)} \circ t^{(2)}$. The resulting arithmetical problem is solved using advanced theory of discriminant forms, and the relation between the properties of the action and the topology of $(X, \mathrm{conj})$ is established using mainly Kalinin's spectral sequence.

\section{Curves on Surfaces}

4.1. Congruences. A topological curve (i.e., disjoint union of embedded circles) $L$ in a topological surface $S$ is said to be totally homologous to zero if the inclusion homomorphism $H_{1}(L) \rightarrow H_{1}(S)$ is trivial. Further, define the genus of a topological surface $S$ to be

$$
g(S)=b_{0}(S)-\frac{1}{2}\left(\chi(S)+b_{0}(\partial S)\right) .
$$

(As there seems to be no commonly accepted definition of genus of a nonorientable or disconnected surface, we choose the one that suits our needs. Certainly, for a connected orientable surface our definition gives the usual genus. In general, the genus is the total number of handles, a cross-cup counted as half a handle.) Note that a surface of genus 0 is a union of spheres with holes, and a surface of genus $1 / 2$ is a union of spheres with holes and one real projective plane with holes. It is also easy to check that if a closed surface $S$ is represented as the union $S_{1} \cup S_{2}$ of two surfaces so that their common boundary $\partial S_{1}=\partial S_{2}$ is totally homologous to zero in $S$, then $\operatorname{dim} \operatorname{Im}\left[\operatorname{in}_{*}: H_{1}\left(S_{i}\right) \rightarrow H_{1}(S)\right]=2 g\left(S_{i}\right), i=1,2$.

4.1.1. Theorem. Let $X$ be a complex surface with real structure, $A$ an ample even real divisor on $X$ with $\mathbb{R} A \neq \varnothing$, and $Y$ the double covering of $X$ branched over $A$. Assume that $H_{1}(X)=0$. The following are necessary conditions for $\mathbb{R} Y_{-}$ to be an $M$ - or $(M-1)$-surface: $X$ is an $M$-surface, $\mathbb{R} A$ lies entirely in a single component of $\mathbb{R} X$, all the other components of $\mathbb{R} X$ belong to $\mathbb{R} X$ - and are covered trivially by $\mathbb{R} Y_{-}$, and $\mathbb{R} A$ is totally homologous to zero in $\mathbb{R} X$. If these conditions are satisfied, then

(1) $\mathbb{R} Y_{-}$is an $M$-surface if and only if $A$ is an $M$-curve and $\mathbb{R} X_{+}$is of genus 0 . In this case $\chi\left(\mathbb{R} X_{+}\right)=\frac{1}{4}[A]^{2} \bmod 8$.

(2) $\mathbb{R} Y_{-}$is an $(M-1)$-surface if and only if either $A$ is an $(M-1)$-curve and $\mathbb{R} X_{+}$is of genus 0 , or $A$ is an $M$-curve and $\mathbb{R} X_{+}$is of genus $1 / 2$. In this case $\chi\left(\mathbb{R} X_{+}\right)=\frac{1}{4}[A]^{2} \pm 1 \bmod 8$. 
Proof. Let $X, Y$, and $A$ be, respectively, an $\left(M-d_{X}\right)$ - and $\left(M-d_{Y}\right)$-surfaces and an $\left(M-d_{A}\right)$-curve. Using the exact sequences of pairs $(\mathbb{R} X, \mathbb{R} A)$ and $\left(\mathbb{R} X, \mathbb{R} X_{+}\right)$, the obvious fact that $\beta_{*}\left(\mathbb{R} X, \mathbb{R} X_{+}\right)=\beta_{*}\left(\mathbb{R} X_{-}\right)$, and the expressions for $\beta_{*}\left(\mathbb{R} Y_{-}\right)$ and $\beta_{*}(Y)$ found, respectively, in the left and right hand sides of the last inequality in 3.1.1 (cf. 2.3.2 and its proof), one obtains

$$
d_{Y}=2 d_{X}+d_{A}+2\left(\beta_{0}(\mathbb{R} X)-c_{-}-1\right)+\left(\delta+\varepsilon_{+}\right)-2 \beta_{1}(X)
$$

where $c_{-}$is the number of closed components of $\mathbb{R} X_{-}$covered trivially, and

$$
\begin{aligned}
\delta & =\operatorname{dim} \operatorname{Im}\left[\operatorname{in}_{*}: H_{1}(\mathbb{R} A) \rightarrow H_{1}(\mathbb{R} X)\right], \\
\varepsilon_{+} & =\operatorname{dim} \operatorname{Im}\left[\operatorname{in}_{*}: H_{1}\left(\mathbb{R} X_{+}\right) \rightarrow H_{1}(\mathbb{R} X)\right]
\end{aligned}
$$

This gives the conditions for $\mathbb{R} Y_{-}$to be an $M$ - or $(M-1)$-surface. The congruences follow from the basic extremal congruences 2.7.1 applied to $\mathbb{R} Y_{-}$and $\mathbb{R} X$.

Note that, unlike the case of plane curves, in general $\mathbb{R} Y_{-}$and $\mathbb{R} Y_{+}$cannot both be close to $M$-surfaces. More precisely, arguing as in the proof of 4.1.1 one obtains (in the obvious notation) $d_{Y_{-}}+d_{Y_{+}}-\beta_{2}(X)=\beta_{2}(X)+\beta_{*}(A)-\left(\beta_{*}\left(\mathbb{R} X_{-}\right)+\right.$ $\left.\beta_{*}\left(\mathbb{R} X_{+}\right)-2 c_{0}\left(\mathbb{R} X_{-}\right)-2 c_{0}\left(\mathbb{R} X_{+}\right)\right)$, and from 3.1.1 it follows that

$$
d_{Y_{-}}+d_{Y_{+}} \geqslant \beta_{2}(X)
$$

Given $X, A$ and $Y$ as above, let us discuss whether the covering $\mathbb{R} Y_{-} \rightarrow \mathbb{R} X_{-}$ is trivial and whether $\mathbb{R} Y_{-}$is orientable. Since $A$ is even and ample, there exists a real curve $E \subset X$ such that $2[E]$ is an odd multiple of $[A]$. (Indeed, one can divide $[A]$ by 2 and replace the result with its large odd multiple in order to make it effective.) Denote by $e$ the class of $[E]$ in ${ }^{\infty} E_{2}(X)$, see A.2. Since $H_{2}(X ; \mathbb{Z})$ is torsion free and $\mathrm{bv}_{2}[\mathbb{R} E]=e$, both $e$ and the class of $[\mathbb{R} E]$ in $H_{1}(\mathbb{R} X)$ are uniquely determined by $[A]$. Next statement follows immediately from the definition of the characteristic class of a double covering and the multiplicative properties of $\mathrm{bv}_{2}$, see A.2.2.

4.1.3. Let $X, A$, and $E$ be as above. Then the characteristic class of the covering $\mathbb{R} Y_{-} \rightarrow \mathbb{R} X_{-}$, considered as a homomorphism $H_{1}\left(\mathbb{R} X_{-}\right) \rightarrow \mathbb{Z}_{2}$, is the restriction to $H_{1}\left(\mathbb{R} X_{-}\right)$of the map $x \mapsto \mathrm{bv}_{2} x \circ e=x \circ[\mathbb{R} E]$.

4.1.4. Corollary. Let $X, A$, and $E$ be as above. If $\mathbb{R} X_{+}$is of genus 0 (or of genus $1 / 2$ ), the projection $\mathbb{R} Y_{-} \rightarrow \mathbb{R} X_{-}$is the orientation double covering if and only if $[\mathbb{R} E]=w_{1}(\mathbb{R} X)$ (respectively, $[\mathbb{R} E]=w_{1}(\mathbb{R} X)+\varkappa c$, where $c$ is the class of a one-sided circle in $\mathbb{R} X_{+}$and $\varkappa \in \mathbb{Z}_{2}$ ).

If $X$ is an $M$-surface, $[\mathbb{R} E]=w_{1}(\mathbb{R} X)$ if and only if $\frac{1}{2}[A]$ is a characteristic element of $H_{2}(X)$.

Note that $\frac{1}{2}[A]$ is a characteristic element of $H_{2}(X)$ if and only if $Y$ is Spin. This follows from the projection formula for $w_{2}$ and the fact that the covering projection induces an epimorphism $H_{2}(Y) \rightarrow H_{2}(X)$, see 2.2.2. Combining 4.1.1 and 4.1.4 with Nikulin's congruence 3.1.5 gives the following result: 
4.1.5. Nikulin's congruence for curves. Let $X$ be a complex surface with real structure, $H_{1}(X)=0$, and $A$ an ample even real divisor on $X$. Assume that

- $X$ is an $M$-surface and $A$ is an $M$-curve,

- $\mathbb{R} A$ is not empty and lies entirely in a single component of $\mathbb{R} X$,

- the other components of $\mathbb{R} X$ belong to $\mathbb{R} X$ - and are orientable,

- $\mathbb{R} A$ is totally homologous to zero in $\mathbb{R} X$,

- $\frac{1}{2}[A]$ is a characteristic element in $H_{2}(X)$.

Assume, further, that for some integer $r \geqslant 0$ the Euler characteristics of all the closed components of $\mathbb{R} X_{-}$are divisible by $2^{r+1}$ and those of the other components are divisible by $2^{r}$ for some integer $r \geqslant 0$. Then:

- if $\mathbb{R} X_{+}$is of genus 0 , then $\chi\left(\mathbb{R} X_{-}\right)=0 \bmod 2^{r+3}$;

- if $\mathbb{R} X_{+}$is of genus $1 / 2$, then $r=0$ and $\chi\left(\mathbb{R} X_{-}\right)= \pm 1 \bmod 8$.

Remark. Complementary Fiedler's congruence, see [Fi1], obtained by means of Rokhlin-Guillou-Marin formula, can also be generalized from plane curves to curves on surfaces.

4.2. Generalizations of Rokhlin's formula of complex orientations. Consider a nonsingular complex surface $X$ with real structure and a dividing real curve $A \subset X$ without real singular points. Denote by $A^{k}$ the $\mathbb{R}$-components of $A$ and assume that each of $A^{k}$ is nonsingular over $\mathbb{C}$. Let $C_{1}, \ldots, C_{n}$ be the connected components of $\mathbb{R} X$, and for each $i=1, \ldots, n$ let $C_{i j}, j=1, \ldots, m_{i}$, be the orientable components of $C_{i} \backslash \mathbb{R} A$. If $C_{i}$ is orientable, fix an orientation on it and endow $C_{i j}$ with the induced orientation; otherwise pick an orientation of $C_{i j}$ arbitrarily. Denote by $\left[C_{i}\right]$ (for orientable $C_{i}$ ) and $\left[C_{i j}\right]$ the fundamental classes in $H_{2}(\mathbb{R} X, \mathbb{R} A ; \mathbb{Z}) \subset H_{2}(\mathbb{R} X, \mathbb{R} A ; \mathbb{Q})$ corresponding to the chosen orientations. Obviously, $\left[C_{i j}\right]$ form a basis of $H_{2}(\mathbb{R} X, \mathbb{R} A ; \mathbb{Z})$ and $\left[C_{i}\right]=\sum_{j=1}^{m_{i}}\left[C_{i j}\right]$ whenever $C_{i}$ is orientable.

Since $A$ is dividing, so are its $\mathbb{R}$-components and, for each $k, \mathbb{R} A^{k}$ separates $A^{k}$ into two halves $A_{ \pm}^{k}$. Let $\left[A_{ \pm}^{k}\right] \in H_{2}(X, \mathbb{R} A ; \mathbb{Z})$ be the class realized by $A_{ \pm}^{k}$ with its complex orientation. Obviously, $\left[A_{+}^{k}\right]+\left[A_{-}^{k}\right]=\left[A^{k}\right]$. (We identify $\left[A^{k}\right],\left[A_{ \pm}^{k}\right]$, etc. with their images under various inclusion and coefficient homomorphisms.) The halves $A_{ \pm}^{k}$ induce a pair of opposite orientations on $\mathbb{R} A^{k}$, called the complex orientations. The corresponding fundamental classes are denoted by $\left[\mathbb{R} A_{ \pm}^{k}\right]$. Let $\left[\mathbb{R} A_{ \pm}\right]=\sum\left[\mathbb{R} A_{ \pm}^{k}\right]$.

Let in: $A \rightarrow X$ be the inclusion. The most straightforward generalization of Rokhlin's formula 1.1 (underlined by V. Zvonilov [Zv2] under slightly stronger assumption) is obtained under the assumption that $\left[\mathbb{R} A_{+}\right]$vanishes in $H_{1}(\mathbb{R} X ; \mathbb{Q})$, which is equivalent to the existence of certain rational numbers $x_{i j}, i=1, \ldots, n$, $j=1, \ldots, m_{i}$, such that

$$
\left[\mathbb{R} A_{+}\right]=\partial \sum x_{i j}\left[C_{i j}\right]
$$

If $C_{i}$ is nonorientable, $x_{i j}$ are uniquely determined by $\mathbb{R} A \subset \mathbb{R} X$ and its complex orientation. If $C_{i}$ is orientable, the class $\sum_{j=1}^{m_{i}} x_{i j}\left[C_{i j}\right]$ is well defined up to a multiple of $\left[C_{i}\right]$. In this case, unless $C_{i}$ is a torus, there is a unique choice of $x_{i j}$ so that $\sum x_{i j} \chi\left(C_{i j}\right)=0$. (If $C_{i}$ is a torus, $\sum x_{i j} \chi\left(C_{i j}\right)$ is determined by $\mathbb{R} A$ and its complex orientation.) 
Denote by $x$ the image of $\sum x_{i j}\left[C_{i j}\right]$ in $H_{2}(X, \mathbb{R} A ; \mathbb{Q})$. There are unique classes $\xi_{+}, \xi_{-} \in H_{2}(X ; \mathbb{Q})$ taken, respectively, into $\left[A_{+}\right]-x,\left[A_{-}\right]+x$ by the relativization homomorphism. It is clear that $\xi_{+}+\xi_{-}=[A]$. Note that, unlike the case of plane projective curves, these classes may not be determined by the complex orientation of $\mathbb{R} A$; thus, they provide a new rigid isotopy invariant of a dividing curve in $X$. According to V. Zvonilov, there are examples when this invariant distinguishes curves with the same complex orientations.

If $A$ is nonsingular, $A_{+} \cap A_{-}=\mathbb{R} A$. In general, $A_{+} \cap A_{-}$differs from $\mathbb{R} A$ by a finite number of points. Denote by $d^{+-}$the number of the points, counted with their multiplicities. Clearly, $0 \leqslant d^{+-} \leqslant \frac{1}{2} \sum_{i<j}\left[A_{i}\right] \circ\left[A_{j}\right]$.

4.2.2. Theorem. Let $A \subset X$ be as above and $\operatorname{in}_{*}\left[\mathbb{R} A_{+}\right]=0$ in $H_{1}(\mathbb{R} X ; \mathbb{Q})$. Then

$$
\xi_{+} \circ \xi_{-}=d^{+-}+\sum_{i, j=1}^{n, m_{i}} x_{i j}^{2} \chi\left(C_{i j}\right)
$$

Furthermore, for each orientable component $C_{i}$ of $\mathbb{R} X$

$$
\xi_{+} \circ\left[C_{i}\right]=-\xi_{-} \circ\left[C_{i}\right]=\sum_{j=1}^{m_{i}} x_{i j} \chi\left(C_{i j}\right) .
$$

Proof. The statement is proved similar to Rokhlin's formula, using the Lagrangian property of $\mathbb{R} X$, (i.e., the fact that the multiplication by $\sqrt{-1}$ transforms the tangent bundle of $\mathbb{R} X$ into its normal bundle with the opposite orientation).

4.2.3. Complement. One has $\xi_{+} \circ[A]=\xi_{-} \circ[A]=\frac{1}{2}[A]^{2}$.

In the case of projective plane $\xi_{+}=\xi_{-}=\frac{1}{2}[A]$, and replacing the left hand side of the first equation in 4.2 .2 by $\frac{1}{4}[A]^{2}$ gives true Rokhlin's formula 1.1 (as well as the Rokhlin-Mishachev formula for curves of odd degree and Rokhlin-Zvonilov-Viro formula for reducible curves). In general one cannot assert that $\xi_{+}=\xi_{-}$and give an implicit form to the above substitute of Rokhlin's formula. From comparing the exact sequences of pairs $(A, \mathbb{R} A)$ and $(X, \mathbb{R} X)$ it follows that $\operatorname{in}_{*}\left[\mathbb{R} A_{+}\right]=0$ and $\xi_{+}=\xi_{-}$(under an appropriate choice of $x_{i j}$ ) if and only if the kernel of the inclusion homomorphism $H_{2}(A, \mathbb{R} A ; \mathbb{Q}) \rightarrow H_{2}(X, \mathbb{R} X ; \mathbb{Q})$ is nontrivial. There is another sufficient condition for $\xi_{+}=\xi_{-}$, which follows from the second equation in 4.2.2 and the obvious fact that $\xi_{+}-\xi_{-}$is $\operatorname{conj}_{*}$-invariant:

4.2.4. Proposition. If $\mathrm{in}_{*}\left[\mathbb{R} A_{+}\right]=0$ in $H_{1}(\mathbb{R} X ; \mathbb{Q})$ and the $(+1)$-eigenspace $H^{+}$ of conj $_{*}$ on $H_{2}(X ; \mathbb{Q})$ is spanned by the fundamental classes of the orientable components of $\mathbb{R} X$, then $\xi_{+}=\xi_{-}$, provided that the coefficients $x_{i j}$ are chosen so that $\sum_{j=1}^{m_{j}} x_{i j} \chi\left(C_{i j}\right)=0$ for each orientable component $C_{i}$ of $\mathbb{R} X$. (Under the hypotheses the last equation holds automatically for all toroidal components.)

If $X$ is Kähler, since $\operatorname{dim} H^{+}=\frac{1}{2}\left(b_{2}(X)+\chi(\mathbb{R} X)\right)-1$, the last hypothesis of 4.2.4 is satisfied, for example, in the following cases:

(1) $b_{2}(X)+\chi(\mathbb{R} X)=2$;

(2) $\mathbb{R} X$ is an orientable $M$-surface, $b_{0}(\mathbb{R} X) \leqslant 2$, and $\mathbb{R} X$ is not a pair of tori;

(3) $\mathbb{R} X$ is an orientable $(M-1)$-surface, $b_{0}(\mathbb{R} X)=1$, and $\mathbb{R} X$ is not a torus. 
Remark: Versions of Arnol'd congruence. Under a stronger assumption in $*[\mathbb{R} A]=$ 0 in $H_{1}(\mathbb{R} X ; \mathbb{Z})$, the coefficients $x_{i j}$ in $(4.2 .1)$ can be chosen integral, and 4.2.2 implies that $\chi(B)=\xi_{+} \circ \xi_{-} \bmod 4$, where $B$ with $\partial B=\mathbb{R} A$ is the union of $C_{i j}$ with $x_{i j}$ odd. On the other hand, if $\operatorname{in}_{*}[\mathbb{R} A]=0$ in $H_{1}(\mathbb{R} X)$ and $B$ is any piece of $\mathbb{R} X$ such that $\partial B=\mathbb{R} A$, then $\chi(B)=\mathcal{P}(\xi)-\chi(A)-\frac{1}{2}[A]^{2} \bmod 4$, where $\mathcal{P}$ is the Pontrjagin square and $\xi \in H_{2}(X)$ is the class of $A_{+}+B$. A piece $B$ with $\partial B=\mathbb{R} A$ exists, e.g., if $\operatorname{in}_{*}[A]=0 \in H_{2}(X)$, and in this case $\chi(B)=\mathcal{P}(\xi) \bmod 4$. If, in addition, $H_{1}(X)=0$, then $X$ is $\mathbb{Z}_{2}$-Galois maximal and $\xi=\frac{1}{2} A+\mathrm{bv}_{2} w_{1}(B)$, where $B$ is a half of $\mathbb{R} X$ corresponding to the double covering; thus, $\mathcal{P}(\xi)$ can be evaluated using the Pontrjagin-Viro form.

Another generalization of Rokhlin's formula, suggested by J.-Y. Welschinger [We1], uses the assumption $\operatorname{in}_{*}[\mathbb{R} A] \in 2 l$ in $_{*}\left[H_{1}(\mathbb{R} A ; \mathbb{Z})\right] \subset H_{1}(\mathbb{R} X ; \mathbb{Z}), l \in \mathbb{Z}$. (In fact, the assumption in [We1] is a bit more general.) It unifies original Rokhlin's formula, Arnol'd congruence, and some of Mikhalkin's congruences [Mik1]. The proof uses the canonical quadratic map $\mathcal{P}_{\text {conj }}: H_{2}(X ; \mathbb{Z} / 2 l \mathbb{Z}) \rightarrow \mathbb{Z} / 4 l \mathbb{Z}$, which extends the reduction of the twisted quadratic form $H_{2}(X ; \mathbb{Z}) \rightarrow \mathbb{Z}, x \mapsto x \circ \operatorname{conj} x$.

Under the above assumption there are integers $x_{i j}, i=1, \ldots, n, j=1, \ldots, m_{i}$, such that $\left[\mathbb{R} A_{+}\right]=\partial \sum x_{i j}\left[C_{i j}\right] \bmod 2 l$. Denote by $x$ the image of $\sum x_{i j}\left[C_{i j}\right]$ in $H_{2}(X, \mathbb{R} A ; \mathbb{Z})$. There are unique classes $\xi_{+}, \xi_{-} \in H_{2}(X ; \mathbb{Z} / 2 l \mathbb{Z})$ taken, respectively, to $\left[A_{+}\right]-x,\left[A_{-}\right]+x$ by the relativization homomorphism. As before, $\xi_{+}+\xi_{-}=[A]$.

4.2.5. Theorem. Given a dividing curve $A$ with $\operatorname{in}_{*}[\mathbb{R} A] \in 2 l \mathrm{in}_{*}\left[H_{1}(\mathbb{R} A ; \mathbb{Z})\right]$, $l \in \mathbb{Z}$, one has

$$
\mathcal{P}_{\text {conj }}\left(\xi_{+}\right)=-\sum_{i, j=1}^{n, m_{i}} x_{i j}^{2} \chi\left(C_{i j}\right) \bmod 4 l .
$$

In some cases, e.g., if $H_{2}(X ; \mathbb{Z})$ is generated by real algebraic curves with known position in respect to $\mathbb{R} A$, the element $\xi_{+}$can be calculated explicitly in terms of the complex orientation of $\mathbb{R} A$. Then 4.2.5 gives some new prohibitions on the complex orientation. Some precise statements can be found in [We1].

4.3. Adjunction formulas. Rokhlin's complex orientation formula is closely related to the Bishop-Lai generalized adjunction formula stated below: they both count the algebraic number of virtual double points and, when they both apply, they lead to the same result; moreover, their geometric proofs go along the same lines.

The traditional adjunction formula for a nonsingular curve $D$ in a nonsingular surface $X$ states that

$$
2 g(D)-2=[D]^{2}+\left[K_{X}\right] \circ[D],
$$

or, equivalently,

$$
\chi(D)+D^{2}=c_{1}(X) \cap D .
$$

If $D$ is singular, one can interpret the defect $\frac{1}{2}\left(\chi(D)+D^{2}-c_{1}(X) \cap D\right)$ as the virtual number of double points of $D$.

The formula can easily be generalized from algebraic curves to immersed real 2manifolds (see [La], [EKh]). Let $F$ be a real oriented compact 2-manifold (possibly disconnected and with boundary) and $f: F \rightarrow X$ an immersion into a complex surface $X$. If $\partial F \neq \varnothing$, we assume that either $f$ has no complex points on $\partial F$ (a 
point $x \in F$ is complex if $d f\left(T_{x} F\right) \subset T_{f(x)} X=\mathbb{C}^{2}$ is a complex line) or all the points of $\partial F$ are complex. Pick a nondegenerate vector field $n: \partial F \rightarrow f^{*}(T X)$ normal to $\partial F$ as follows: if $f$ has no complex points on $\partial F$, let $n: \partial F \rightarrow T F \subset f^{*}(T X)$ be normal to $\partial F$ in $F$; otherwise, $T F \subset f^{*}(T X)$ is a complex line subbundle of $f^{*}(T X)$ over $\partial F$ and $n: \partial F \rightarrow f^{*}(T X)$ is taken normal to $T F$.

Denote by $\nu(f) \in \mathbb{Z}$ the obstruction to extending $\sqrt{-1} n$ to a vector field normal to $F$, and by $c_{1}(f) \in \mathbb{Z}$, the obstruction to extending $(\tau, n)$, where $\tau$ is a nondegenerate tangent field on $\partial F$, to a complex 2 -frame field in $f^{*}(T X)$ over $F$. The latter is the Maslov index of the framing with respect to to the membrane $F$. If $\partial F=\varnothing$, then $\nu(f)$ is the normal Euler number of $F$ in $f^{*}(T X)$ and $c_{1}(f)=c_{1}(X) \cap f_{*}[F]$. In this notation, the Bishop-Lai formula states

$$
\chi(F)+\nu(f)-c_{1}(f)=2 d_{-}(f), \quad \chi(F)+\nu(f)+c_{1}(f)=2 d_{+}(f),
$$

where $d_{ \pm}(f)$ are determined by the complex points of $f$. More precisely, if $f$ is generic, then $d_{ \pm}(f)=e_{ \pm}(f)-h_{ \pm}(f)$, where $e_{ \pm}$(respectively, $h_{ \pm}$) are the numbers of positive and negative elliptic (respectively, hyperbolic) points of $f$. In general, $d_{+}(f)$ and $d_{-}(f)$ can be defined as the intersection numbers of the image of $F$ in the Gauss fibration of oriented real 2-planes in $f^{*}(T X)$ with the cycle of complex lines equipped by their complex or, respectively, anti-complex orientation. If a component of $\partial F$ consists of complex points, it is first shifted off the cycle in the tangent directions $\tau^{*} \otimes n+\sqrt{-1} \tau^{*} \otimes \sqrt{-1} n$ determined by the real 2-planes $(\tau, n)$. If $f$ is generic, $e_{+}$and $h_{+}$(respectively, $e_{-}$and $h_{-}$) are the numbers of the positive and negative intersection points of $F$ with the complex (respectively, anti-complex) orientation cycle. A definition of elliptic and hyperbolic points in local coordinates can be found, e.g., in [EKh].

4.3.3. The definition of $\nu(f)$ and $c_{1}(f)$ and the Bishop-Lai formula extend to a wider class of surfaces: one can assume that $F$ and $f: F \rightarrow X$ are sewed (along boundary components) of surfaces $F_{i}$ and immersions $f_{i}: F_{i} \rightarrow X$ satisfying the above conditions, so that any two adjacent pieces are normal in $X$. (We do not impose any requirements on the sewn boundary fields, as we do not use additivity relations.) Note also that, if $F$ is closed, the algebraic number $i(f)$ of virtual double points of $f$ is given by

$$
i(f)=\frac{1}{2}\left(\left(f_{*}[F]\right)^{2}-\nu(f)\right)=\frac{1}{2}\left(\chi(F)+\left(f_{*}[F]\right)^{2} \pm c_{1}(f)\right)-d_{ \pm}(f) .
$$

The following statement is a straightforward consequence of (4.3.2).

4.3.5. Let $F$ be a closed oriented real 2-manifold, $X$ a complex surface, and $f: F \rightarrow$ $X$ a piecewise smooth map as in 4.3.3 whose image has no negative complex points. Then

$$
\chi(F)+\nu(f)=c_{1}(f) .
$$

4.3.6. Corollary. In the notation of 4.2 .2 one has

$$
\xi_{ \pm}^{2}=\frac{1}{2}[A]^{2}-d^{+-}-\sum_{i, j=1}^{n, m_{i}} x_{i j}^{2} \chi\left(C_{i j}\right) .
$$

Proof. Apply 4.3.5 twice: first to $\xi$ (or to each of its smooth pieces), and then to $A$. (Certainly, the same result follows from 4.2 .2 and 4.2 .3 , cf. the beginning of this section.)

In the case of plane curves 4.3.6 turns into Rokhlin's formula. 
4.4. An application of the adjunction inequality. Let $A \subset X$ be as in 4.2 . Assume that $\operatorname{in}_{*}\left[\mathbb{R} A_{+}\right]=0 \in H_{1}(\mathbb{R} X ; \mathbb{Z})$ and denote by $x_{i j}$ the integers resolving (4.2.1). The cycle $\xi_{+}$, see 4.2 , can be represented as $f_{*}[F]$ for some map $f: F \rightarrow X$ and some surface $F$ sewed of $A_{+}$and $\left|x_{i j}\right|$ copies of $C_{i j}$. Such a cycle has no negative complex points and, in view of (4.3.4) and 4.3.5, the number $i_{+}\left(f^{\prime}\right)$ of positive double points of a generic bifurcation $f^{\prime}$ of $f$ satisfies the adjunction inequality

$$
i_{+}\left(f^{\prime}\right) \geqslant \frac{1}{2}\left(\chi(F)+\left(f_{*}[F]\right)^{2}-c_{1}(f)\right) .
$$

The results obtained using the inequality are weaker than Rokhlin's formula (which, in this case, is equivalent to the Bishop-Lai formula). However, as it was observed by G. Mikhalkin [Mik2], new prohibitions on reducible real curves can be obtained applying the adjunction inequality to similar mixed cycles with the orientation on some of holomorphic pieces reversed. (For the sake of simplicity we ignore the somewhat more cumbersome case where the irreducible components have real intersection points; on the other hand, we treat curves on arbitrary Kähler surfaces.)

Thus, fix an integer $s \geqslant 1$ and let $f^{\prime}: F \rightarrow X$ be identical to $f$ over all $C_{i j}$ and all $A_{+}^{k}$ with $k>s$ and equal to conj $\circ$ over all $A_{+}^{k}$ with $1 \leqslant k \leqslant s$. Denote by $d_{1}, d_{2} \in H_{2}(X ; \mathbb{Z})$ the elements realized by $\bigcup_{k>s} A_{k}$ and $\bigcup_{k \leqslant s} A_{k}$, respectively, and let $x^{++}$be the algebraic intersection number of $\bigcup_{k>s} A_{+}^{k}$ and $\bigcup_{k \leqslant s} A_{+}^{k}$ (recall that, due to the assumption made, see 4.2 , the boundaries of the two chains are disjoint).

4.4.1. Theorem. Let $A \subset X$ be as in 4.2 and $\operatorname{in}_{*}\left[\mathbb{R} A_{+}\right]=0$ in $H_{1}(\mathbb{R} X ; \mathbb{Z})$. If $X$ is Kähler and $\omega \cap\left(d_{1}-d_{2}\right)>0$, where $\omega$ is the Kähler class, then

$$
j_{-} \geqslant x^{++}+\frac{1}{2}\left(c_{1}(X) \cap d_{2}-d_{1} \circ d_{2}\right),
$$

where $j_{-}=\sum \frac{1}{2}\left|x_{i j}\right|\left(\left|x_{i j}\right|-1\right)$, the sum taken over all $\left|x_{i j}\right| \geqslant 2$ corresponding to the components $C_{i j}$ homeomorphic to a disc.

Proof. The inequality is the difference between 4.3 .5 applied to $f$ and the adjunction inequality applied to the usual Lagrangian perturbation of $f^{\prime}$, which provides an immersion with $j_{-}+\frac{1}{2}\left(d_{1} \circ d_{2}\right)-x^{++}$negative double points.

4.4.2. Corollary. Let $X, d_{1}, d_{2}$ be as above and $\omega \cap\left(d_{1}-d_{2}\right)>0$ (see 4.4.1). Assume that the $(+1)$-eigenspace of $\operatorname{conj}_{*}$ on $H_{2}(X ; \mathbb{Q})$ is spanned by the fundamental classes of the orientable components of $\mathbb{R} X$ and that for every $k$ each connected component of $\mathbb{R} A^{k}$ bounds a disc. Then

$$
j_{-} \geqslant z^{+}-z^{-}+\frac{1}{2} c_{1}(X) \cap d_{2}-\frac{1}{4}\left(d_{1} \circ d_{2}\right),
$$

where $z^{ \pm}$are the numbers of positive and negative injective pairs of ovals such that one of the ovals is in $A_{k}$ with $k \leqslant s$ and the other one, in $A_{k}$ with $k>s$. (In order to define injective pairs for each connected component of $\mathbb{R} A$ one picks and fixes a disk bounded by this component.)

Proof. Construct the Rokhlin cycles by filling $\bigcup_{k>s} A_{+}^{k}$ and $\bigcup_{k \leqslant s} A_{-}^{k}$ with disks. Then $x^{++}$is found by calculating the intersection of the cycles in two ways: homologically (applying 4.2.4) and geometrically. 
The following example of application of 4.4.2 (combined with Rokhlin's formula) is also due to Mikhalkin. Let $A$ be a real plane curve of degree $2 k$ with the arrangement $\left\langle\frac{3}{2} k(k-1)\right\rangle \sqcup 1\left\langle\frac{1}{2}(k-1)(k-2)\right\rangle$ (Harnack arrangement), and $C$ a real conic with $\mathbb{R} C$ disjoint from $\mathbb{R} A$. Then at most $(k-2)$ of the interior ovals of $\mathbb{R} A$ are inside the oval of $\mathbb{R} C$.

4.5. Orevkov's formula. S. Orevkov [Or1] observed that, if a plane $M$-curve of degree $d$ has a nest of depth $k-1, k=[d / 2]$, Rokhlin's formula splits into two separate identities. Under the assumption the nonempty ovals are pairwise nested and, thus, are divided into positive and negative (e.g., with respect to the outermost oval of the nest). Denote by $\pi_{+}^{+}$and $\pi_{+}^{-}$(respectively, $\pi_{-}^{+}$and $\pi_{-}^{-}$) the number of positive and negative injective pairs whose inner oval is empty and outer oval is positive (respectively, negative).

4.5.1. Orevkov's formula. For any plane $M$-curve of degree $2 k$ with a nest of depth $k-1$ one has

$$
\pi_{+}^{-}-\pi_{+}^{+}=k_{+}^{2}, \quad \pi_{-}^{+}-\pi_{-}^{-}=k_{-}^{2},
$$

where $k_{+}$and $k_{-}$are the number of positive and negative nonempty ovals.

The formula above and a similar formula for curves of odd degree give a new information on the complex orientation in degrees $\geqslant 7$. Both the formulas extend to nonmaximal dividing curves.

The original proof (see [Or1]) is based on counting the self-linking numbers of the braids cut by $\mathbb{C} A$ on the boundary of the two 4-balls formed by the imaginary points of the imaginary lines through a point in an innermost oval of the nest. J.-Y. Welschinger [We2] observed that Orevkov's formula can also be obtained from the adjunction formula applied to appropriate mixed singular cycles and generalized the formula to curves on conic bundles. For simplicity, we state his result for geometrically ruled surfaces, i.e., conic bundles without singular fibers.

Let $\pi: X \rightarrow B$ be a holomorphic equivariant map of a real surface to a real curve. Assume that $B$ is a dividing curve and each fiber $F$ of $\pi$ is a smooth irreducible rational curve. Assume, in addition, that $\pi$ has a real section $E$. The fundamental classes of $F$ and $E$ form a basis in $H_{2}(X ; \mathbb{Z})$.

Denote by $B_{1}, \ldots, B_{p}$ the connected components of $\mathbb{R} B$ and by $X_{1}, \ldots, X_{p}$, their pull-backs in $\mathbb{R} X$. (Each $X_{j}$ is a torus or a Klein bottle.) Pick a half $B_{+}$of $B$ and equip $\mathbb{R} B$ with the corresponding complex orientation.

Let $A \subset X$ be a nonsingular irreducible dividing real $(M-2 r)$-curve with a contractible oval on each real component of $X$ (the latter assumption is not essential for 4.5.2). Pick a half $A_{+}$of $A$ and equip $\mathbb{R} A$ with the corresponding complex orientation. For each $i, 1 \leqslant i \leqslant p$, consider the components of $\mathbb{R} A$ which project to $B_{i}$ with a positive (respectively, negative) degree and denote by $n_{i}^{+}$(respectively, $n_{i}^{-}$) their total degree. For each $i$ there is a positive integer $k_{i}$ such that $n_{i}^{+}+n_{i}^{-}+$ $2 k_{i}=[A] \circ[F]$. If $k_{i}=1$ for each $i, 1 \leqslant i \leqslant p$, we say that $A$ is a curve with a deep nest. Clearly, for a curve with a deep nest the weights $n^{+}=n_{i}^{+}$and $n^{-}=n_{i}^{-}$do not depend on $i$.

From now on assume that $A$ is a curve with a deep nest. Then each real fiber of $\pi$ meets $A$ in at most two imaginary points. If a real fiber $F$ does meet $A$ at two imaginary points, pick a point $x \in \mathbb{R} F$ not in $E$. Such a choice can be made continuously. Moreover, if all the turn points (i.e., the points of tangency of $\mathbb{R} A$ and a fiber) lie outside $E$, there is a canonical choice: trace the circle $\gamma$ in $F$ through 
the two imaginary points and the point of $E$ and take for $x$ the intersection point of $\gamma$ and $\mathbb{R} F$ other than the point of $E$. The chosen points form a finite collection of $\operatorname{arcs} S_{j}$, each connecting two turn points. The union of $S_{j}$ is completed to a closed, not necessarily connected or simple, curve $\Gamma^{+}$(respectively, $\Gamma^{-}$) by arcs of $\mathbb{R} A$ projecting to $\mathbb{R} B$ with positive (respectively, negative) local degree. The complex orientation of $\mathbb{R} A$ defines a coorientation of $S_{j}$ at its boundary points; it extends to a continuous coorientation of $S_{j}$. Denote the extended coorientation by $\omega$. Without lost of generality one can assume that the intersection points of the interiors of $S_{j}$ and $\mathbb{R} A$ are transversal, and attribute to each point sign +1 if $\omega$ coincides with the orientation of $\mathbb{R} A$ and -1 otherwise. Let $\Lambda^{ \pm}$be the sum of the signs over all the double points of $\Gamma^{ \pm}$.

To make $\Lambda^{ \pm}$more invariant, remove the zig-zags on $\mathbb{R} A$ (each zig-zag changes one of $\Lambda^{ \pm}$by $n^{ \pm}-1$ or 1 , depending on whether it crosses $\mathbb{R} E$ or not). Adjusted so, $\Lambda^{ \pm}$are preserved by the isotopies of nonorientable components of $\mathbb{R} X$ identical on $\mathbb{R} E$ and on each component of $\mathbb{R} A$ intersecting $\mathbb{R} E$. More generally, $\Lambda^{ \pm}$are preserved by an isotopy of nonorientable components of $\mathbb{R} X$ identical on $\mathbb{R} E$ if during the isotopy the turn points do not cross $\mathbb{R} E$ (each crossing changing both $\Lambda^{ \pm}$by $n^{ \pm}$). An isotopy of an orientable component of $\mathbb{R} X$ preserves $\Lambda^{ \pm}$, e.g., if during the isotopy (as well as at the initial position) $\mathbb{R} A$ does not intersect $\mathbb{R} E$.

4.5.2. Theorem. Under the above assumption there are integers $\mu^{ \pm}, g^{ \pm}, a^{ \pm}$such that

$$
\Lambda^{ \pm}+n^{ \pm} a^{ \pm}+\mu^{ \pm}-g^{ \pm}=\frac{1}{2}\left(\chi(B)-n^{ \pm} E^{2}\right)\left(n^{ \pm}+1\right)
$$

and

$$
\begin{gathered}
g^{ \pm} \geqslant c^{ \pm}, \quad g^{+}+g^{-}+2-\mu^{+}-\mu^{+}=c^{+}+c^{-}+2 r, \\
\mu^{ \pm} \geqslant 1, \mu^{+}+\mu^{-} \leqslant p+1, \\
a^{+}+a^{-}=a, \quad a^{ \pm}+E^{2}\left(n^{ \pm}+1\right)=\mathbb{R} E \circ \Gamma^{ \pm} \bmod 2, \\
\left|\mathbb{R} E \cap \Gamma^{ \pm}\right| \leqslant a+E^{2}\left(n^{ \pm}+1\right) \leqslant a^{ \pm}+E^{2} b-\left|\mathbb{R} E \cap \Gamma^{\mp}\right|,
\end{gathered}
$$

where $a[F]+b[E]=[A]$ and $c^{+}, c^{-}$are the numbers of the components of $\mathbb{R} A$ projecting to a component of $\mathbb{R} B$ with a positive (respectively, negative) degree.

Proof. Consider the surface $W^{ \pm}=\left(A^{ \pm} \cap \pi^{-1}\left(B^{+}\right)\right) \cup\left(A^{\mp} \cap \pi^{-1}\left(B^{-}\right)\right)$and fill its holes with embedded discs contained in the pull-back of $\mathbb{R} B$, with the segments $S_{j}$ as diameters (e.g., one can use arcs of the circles described in the construction of $S_{j}$ ). The statement follows from the adjunction formula 4.3.5 applied to the resulting singular cycle $\Sigma^{ \pm}$, using $\nu=\left(\Sigma^{ \pm}\right)^{2}+2 \Lambda^{ \pm}, \Sigma^{ \pm} \circ F=n^{ \pm}+1$, introducing $a^{ \pm}$by $\left[\Sigma^{ \pm}\right]=a^{ \pm}[F]+\left(n^{ \pm}+1\right)[E]$ and taking for $g^{ \pm}$and $\mu^{ \pm}$respectively the genus and the number of connected components of $W^{ \pm}$.

4.5.3. Corollary. If $A$ as in 4.5.2 does not intersect $E$, one has

$$
\Lambda^{ \pm}+\mu^{ \pm}-g^{ \pm}=\frac{1}{2}\left(\chi(B)+E^{2} n^{ \pm}\right)\left(n^{ \pm}+1\right) .
$$

Remark. To obtain 4.5.1 from 4.5.3, blow up a point in the innermost oval of a nest of depth $k-1$; then one has $\chi(B)=2, E^{2}=-1, \mu^{ \pm}=1, g^{ \pm}=K_{ \pm}$, and $\Lambda^{ \pm}=\Pi_{ \pm}^{-}-\Pi_{ \pm}^{+}$, where $K$ and $\Pi$ differ from $k$ and $\pi$ in 4.5 .1 by the fact that the innermost oval chosen contributes to $K$ and does not contribute to $\Pi$, whereas it does not contribute to $k$ and contributes to $\pi$. 
4.6. The Bezout Theorem. The Bezout theorem plays an important rôle in theory of real plane projective algebraic curves. For an arbitrary surface $X$, in its complex form this famous theorem states that given two curves $A, B \subset X$ without common component, the number of intersection points of $A$ and $B$, counted with their multiplicities, equals $[A] \circ[B]$. An important fact is that the multiplicity of an intersection point of two analytic curves without common components is a positive integer. This gives the following real version of the Bezout theorem (cf. a remark in A.2): if $X$ is real and $A, B \subset X$ are real curves without common components, then the number of intersection points of $\mathbb{R} A$ and $\mathbb{R} B$, counted with their multiplicities, does not exceed $[A] \circ[B]$ and is congruent to $[A] \circ[B] \bmod 2$.

One of the typical applications of the Bezout theorem is tracing an auxiliary curve $D$ through a certain number of appropriately chosen points and estimating the number of intersection points of $\mathbb{R} D$ and the given curve $\mathbb{R} A$ (it is in this way that Harnack obtained his bound for the number of real components of plane curve). Sometimes one also needs to know that $\mathbb{R} D$ is connected or, more generally, that all the intersection points belong to the same component of $\mathbb{R} D$. The simplest way to assure this is to consider rational curves.

Thus, in order to apply the Bezout theorem on an arbitrary surface $X$ one needs to know, first, whether a given class $x \in H_{2}(X ; \mathbb{Z})$ can be realized by a (formal linear combination of) analytic curves, second, the dimension of the space of such curves, and third, the genus of such curves. The answer to the first question in the case when $X$ is projective is given by the Lefschetz-Hodge theorem (see [KS]): a homology class $x \in H_{2}(X ; \mathbb{Z})$ can be realized by a formal linear combination of algebraic curves on $X$ if and only if the class Poincaré dual to $x$ belongs to $H^{1,1}(X)$ (see 2.4). Though the dimension $h^{1,1}(X)=\operatorname{dim} H^{1,1}(X)$ for surfaces is a topological invariant, $h^{1,1}(X)=\sigma^{-}(X)+1$, the position of $H^{1,1}$ in $H^{2}(X ; \mathbb{Z})$ depends on the complex structure on $X$ and may change when $X$ varies within its deformation type. Thus, in general one can only assert that the rational multiples of the hyperplane section class (those which are still integral classes) can be realized by combinations of algebraic curves. However, if $h^{1,1}(X)=b_{2}(X)$, i.e., $\sigma^{+}(X)=1$, all classes in $H_{2}(X ; \mathbb{Z})$ are algebraic. Examples are rational surfaces and Enriques surfaces.

The dimension of a linear system $|D|$ can, in certain cases, be estimated using the Riemann-Roch theorem:

$$
\operatorname{dim}|D|+\operatorname{dim}\left|K_{X}-D\right| \geqslant \frac{1}{2}\left([D]^{2}-\left[K_{X}\right] \circ[D]\right)+p_{a}(X)-1,
$$

where $p_{a}(X)=\frac{1}{2}\left(\sigma^{+}(X)-b_{1}(X)+1\right)$ is the arithmetic genus of $X$. If $D$ is ample (recall that for an effective divisor on a surface this is equivalent to $[D]^{2}>0$ and $[D] \circ\left[D^{\prime}\right] \geqslant 0$ for all irreducible components of $\left.D\right)$, then $\left|K_{X}-D\right|=\varnothing$ and in 4.6.1 equality is attained:

$$
\operatorname{dim}|D|=\frac{1}{2}\left([D]^{2}-\left[K_{X}\right] \circ[D]\right)+p_{a}(X)
$$

The following statement is an easy consequence of (4.6.2).

4.6.3. Proposition. Let $D$ be an ample real divisor and $s_{D}=\frac{1}{2}\left([D]^{2}-\left[K_{X}\right]\right.$ 。 $[D])+p_{a}(X)$. Then through any collection of $s_{D}$ points one can trace a real curve 
in $|D|$. Moreover, for any $\left(s_{D}-s\right)$-point set $S, s \geqslant 0$, the real curves in $|D|$ containing $S$ form a real linear system of dimension $\geqslant s$.

Unfortunately, this result is not sufficient even to generalize the well known Hilbert bounds to the higher numbers of nests. The difficulty is that the number of connected components of a curve given by 4.6 .3 is not easy to control. In general one can only claim that this number is between 1 and $2+\frac{1}{2}\left([D]^{2}+\left[K_{X}\right] \circ[D]\right)$ i.e., the Harnack bound. Thus, it would be useful to know, in addition, through what number of points one can trace a connected component of a curve in $|D|$. (This and other related questions, more or less explicitly raised in [R3], seem to be of an independent interest, regardless of bounds on the nests).

Denote by $c(D)$ the maximal number $c$ such that through any $c$ points of $\mathbb{R} X$ in general position one can trace a real curve in $|D|$ with connected real point set. Denote, further, by $c^{\prime}(D)$ the maximal number $c$ such that through any $c$ points of $\mathbb{R} X$ (not necessarily in general position) one can trace a connected component of the real point set of a real curve in $|D|$.

4.6.4. Proposition. $c^{\prime}(D) \geqslant c(D)$ for any divisor $D$.

4.6.5. Lemma. Let $\left\{F_{n}\right\}$ be a sequence of continuous functions on a Hausdorff compact space $Y$ converging uniformly to a function $F$. If the zero set $Z_{n}$ of $F_{n}$ is connected for each $n$, then the zero set $Z$ of $F$ has a connected component which contains $\bigcap_{n} Z_{n}$.

Proof. Assume that $\bigcap_{n} Z_{n} \neq \varnothing$. It is contained in $Z$. Pick a point $z \in \bigcap_{n} Z_{n}$ and an open-closed subset $C$ of $Z$ containing $z$. Since $Z$ is closed in $Y$, so are $C$ and $Z \backslash C$ and, hence, they are separated by a neighborhood $U$ (i.e, $U \supset C$ and $\mathrm{Cl} U \cap(Z \backslash C)=\varnothing)$.

Since the convergence $F_{n} \rightarrow F$ is uniform, there is an integer $N$ such that $Z_{n} \subset U \cup(Y \backslash \mathrm{Cl} U)$ for all $n>N$. Then, since all $Z_{n}$ are connected and intersect $C \subset U$, each $Z_{n}$ with $n>N$ is contained in $U$ and, hence, $\bigcap_{n} Z_{n}$ is contained in $C=U \cap Z$. It remains to notice that in a compact Hausdorff space the intersection of all open-closed subsets containing a given point is the component of the point.

Proof of 4.6.4. Let $c$ be a number such that through any generic set of $c$ points of $\mathbb{R} X$ one can trace a real algebraic curve in $|D|$ with connected real point set. We need to prove that an arbitrary $c$-point set in $\mathbb{R} X$ is contained in a connected component of a real curve in $|D|$.

Consider a $c$-point set $\Sigma \subset \mathbb{R} X$. By the assumption, there is a sequence $\left\{\Sigma_{n}\right\}$ of $c$-point sets in $\mathbb{R} X$ converging to $\Sigma$ and such that each $\Sigma_{n}$ is contained in a real connected curve $\mathbb{R} A_{n}$ in $|D|$. Let $\xi$ be the $\mathbb{R}$-bundle over $\mathbb{R} X$ corresponding to $|D|$. Its tensor square, as the square of any $\mathbb{R}$-bundle, is trivial. Let $F_{n}, n \geqslant 1$, be a real section of the $\xi$ representing $A_{n}$. The space of sections is finite dimensional. Hence, after a proper scaling $\left\{F_{n}\right\}$ contains a convergent subsequence. As usual, one can assume that $\left\{F_{n}\right\}$ converges itself. It remains to apply Lemma 4.6 .5 to $F_{n}^{2}$, considered as functions on $\mathbb{R} X$.

The following additivity property is an immediate consequence of the definitions.

4.6.6. Proposition. For any divisors $D_{1}, D_{2}$ one has

$$
c\left(D_{1}+D_{2}\right) \geqslant c\left(D_{1}\right)+c\left(D_{2}\right)-1 \text { and } c^{\prime}\left(D_{1}+D_{2}\right) \geqslant c^{\prime}\left(D_{1}\right)+c^{\prime}\left(D_{2}\right)-1 .
$$


Some other related problems are also worth mentioning. First, it is natural to consider curves with a bounded number of components. Then $c(D)$ becomes the first member $c_{1}(D)$ of a larger family of characteristics $c_{\leqslant k}(D)$, defined similar to $c(D)$ with connected curves replaced by curves with at most $k$ connected components. Second, one can consider the minimax $\nu(r, D)$ and maximin $\mu(r, D)$ of the number of connected components in a linear subsystem of given dimension $r$ in $|D|$. Another possibility is to consider other topological characteristics of a linear system, say, typical Morse numbers, etc.

4.6.7. Marin's Bound. If $D$ is very ample and $\chi(X)+D^{2}$ is odd, any generic pencil of real curves in $|D|$ contains at least one $(M-j)$-curve with $j \geqslant 3$.

This result can be restated as $\mu(1, D) \leqslant M(D)-3$, where $M(D)$ is the Harnack number $2+\frac{1}{2}\left([D]^{2}+\left[K_{X}\right] \circ[D]\right)$.

4.6.8. Klein's statement. If a real curve of type I undergoes a Morse surgery through a nondegenerate double point, its number of connected components can not increase.

Note that the number of connected components of the curve does not need to change, cf. 4.6.9 below. In other words, Klein's statement means that for any $q$ the number of connected components, considered as a piecewise constant function on the space $\mathbb{R} C_{q}$ of curves of degree $q$, attains a 'local maximum' at each component of $\mathbb{R} C_{q} \backslash \mathbb{R} D_{q}$ (where $\mathbb{R} D_{q}$ is the hypersurface of singular curves) formed by real curves of type I. Using complex orientations one can check that, in addition to 4.6.7 and 4.6.9, there is no wall (i.e., principal stratum of $\mathbb{R} D_{q}$ ) adjacent to two components formed by curves of type I. (There is an exception: $q=2$ and the wall corresponding to turning the oval inside out; however, this wall is not adjacent to two different components!)

Remark. There are extensions of 4.6 .8 to surfaces (see [V3]) and to hypersurfaces in a projective space (see $[\mathrm{Ka}]$ ).

Proof of 4.6.8. Let $A_{t}, t \in[-\varepsilon, \varepsilon]$, be a family of real curves such that: $A_{0}$ is a curve with a double nondegenerate point, $A_{<0}$ are of type I, and the number of connected components decreases from $\mathbb{R} A_{>0}$ to $\mathbb{R} A_{<0}$. The last assumption implies that the singular connected component of $\mathbb{R} A_{0}$ lifted to the normalization of $A_{0}$ is a topological circle. In particular, the number of real components of the normalization of $A_{0}$ is equal to the number of components of $\mathbb{R} A_{<0}$. On the other hand, the genera of $A_{0}$ and $A_{\neq 0}$ differ by $1: g\left(A_{0}\right)=g\left(A_{\neq 0}\right)-1$. Thus, due to, e.g., Klein's congruence (which states that the number of real components of a dividing curve has the same parity as the genus, cf. Introduction), $A_{0}$ is not a curve of type I. Therefore, two conjugate points in $A_{0} \backslash \mathbb{R} A_{0}$ can be joined by a path in $A_{0} \backslash \mathbb{R} A_{0}$. Hence, such a path exists also in each close curve $A_{t}, t \neq 0$, which contradicts to the assumption that the curves $A_{t}$ with $t<0$ are of type I.

For $(M-2)$-curves Klein's statement can be strengthened.

4.6.9. Proposition. If two $(M-2)$-curves are obtained from each other by a Morse surgery through a double nondegenerate point, one of them is of type I and the other one is of type II.

Proof. Denote by $A_{t}, t \in[-\varepsilon, \varepsilon]$, a family of real curves such that: $A_{0}$ is a curve with a double nondegenerate point and $A_{\neq 0}$ are $(M-2)$-curves. Since the number of 
connected components does not change from $\mathbb{R} A_{>0}$ to $\mathbb{R} A_{<0}$, the singular connected component of $\mathbb{R} A_{0}$ lifted to the normalization of $A_{0}$ turns into two topological circles. In particular, the number of real components of the normalization of $A_{0}$ is greater by 1 than the number of components of $\mathbb{R} A_{t}$ with $t \neq 0$. On the other hand, $g\left(A_{0}\right)=g\left(A_{\neq 0}\right)-1$. Thus, the normalization is an $M$-curve and, hence, it is of type I, and so is its perturbation coherent with the complex orientation (while the other one is of type II).

Proof of 4.6.7. Consider graph $\Gamma$ with 4 vertices representing $M-,(M-1)$-, and $(M-2)$-curves of type I and $(M-2)$-curves of type II. Two vertices are connected by an edge if there are adjacent curves representing them. Due to 4.6.8 and 4.6.9, $\Gamma$ has only three edges: they connect $M$ and $M-1, M-1$ and $M-2$ of type II, and $M-2$ of type II and $M-2$ of type I. In particular, $\Gamma$ supports no cycle of odd length.

A generic pencil of real curves in $|D|$, considered as a line in the corresponding projective space, intersects the discriminant hypersurface of singular curves transversally, the intersection points representing surgeries of the real parts of the curves. The degree of the discriminant has the same parity as $\chi(X)+D^{2}$. Hence, if the pencil does not contain an $(M-j)$-curve with $j \geqslant 3$, it defines an odd length cycle in the graph constructed above, which is a contradiction.

To conclude this section we apply the above results to derive some bounds on nests. The first theorem is stated so that it depends only on a lower bound for $c^{\prime}(D)$.

4.6.10. Proposition. If a real curve $C$ has $c \leqslant c^{\prime}(D)$ disjoint nests of depths $h_{1}, \ldots, h_{c}$, so that no oval of the nests of depth $>1$ envelopes all the other ovals of the nests, then

$$
h_{1}+\cdots+h_{c}+\left[\frac{1}{2}\left(c^{\prime}(D)-c\right)\right] \leqslant \frac{1}{2} C \circ D .
$$

In particular, if $C$ has $c \leqslant c^{\prime}(D)-1$ disjoint nests of depths $h_{1}, \ldots, h_{c}$, so that no oval of the nests of depth $>1$ envelopes all the other ovals of the nests, and

$$
h_{1}+\cdots+h_{c}+\left[\frac{1}{2}\left(c^{\prime}(D)-c\right)\right]=\left[\frac{1}{2} C \circ D\right],
$$

then the union of the nests exhausts the whole curve.

Proof. Take a point on (or inside, if $h>1$ ) the innermost oval of each nest and $c^{\prime}(D)-c$ more points on one of these ovals. Trace a connected component of a real curve of $|D|$ trough the $c^{\prime}(D)=c+\left(c^{\prime}(D)-c\right)$ points obtained. It intersects the original curve in at least $2\left(h_{1}+\cdots+h_{c}\right)+r$ points, where $r=2\left[\frac{1}{2}\left(c^{\prime}(D)-c\right)\right]$. Now the bound follows from the Bezout theorem, and the extremal property is its direct consequence.

If the union of the nests does not exhaust the real part of the curve, the estimate can be sharpened slightly, by choosing the additional $c^{\prime}(D)-c$ points on an oval not in the nests.

4.6.11. Proposition. Let a real curve $C$ have $s=\frac{1}{2} D \circ(D-K)-l$ nests outside each other. If $s>\mu(l, D)$, then there are $s-\mu(l, D)+1$ nests (among the given ones) whose total weight is at most $\frac{1}{2} C \circ D-(\mu(l, D)-1)$.

Proof. Take a point on the innermost oval of each nest and consider the linear system of curves in $|D|$ through these points. Its dimension is at least $l$, and by 
the definition of $\mu(l, D)$ the system contains a curve $D_{0}$ with $\leqslant \mu(l, D)$ connected components. Hence, at least $s-\mu(l, D)+1$ nests contribute at least twice their depth to the intersection with $D_{0}$. Each other nest contributes at least two, by its innermost oval. It remains to apply the Bezout inequality.

4.6.12. Corollary. If a curve $C$ has $\frac{1}{2} D \circ(D-K)$ nests outside each other, then among these nests there are $r=-D \circ K-1$ nests whose total weight is at most $\frac{1}{2} C \circ D-\frac{1}{2} D \circ(D+K)-1$.

Proof. This is a special case of 4.6 .11 corresponding to $l=0$. Indeed, $\mu(0, q)=$ $\frac{1}{2} D \circ(D+K)+2$ and $r=\frac{1}{2} D \circ(D-K)-\mu(0, D)+1$.

4.7. Plane curves through given points. In the case of plane curves a linear system $|D|$ is determined by the degree $q$ of $D$. Thus, one deals with sequences $\{c(q)\},\left\{c^{\prime}(q)\right\}$. Their first four terms are known.

4.7.1. Proposition. One has $c(1)=c^{\prime}(1)=2, c(2)=c^{\prime}(2)=5, c(3)=c^{\prime}(3)=8$, and $c(4)=c^{\prime}(4)=13$.

Note that, in fact, in any real pencil of cubics there is a real cubic with the connected real part.

Proof. The real point set of any real curve of degree 1 or 2 is connected. Due to 4.6 .4 it remains to prove that

$$
8 \leqslant c(3), c^{\prime}(3)<9, \quad 13 \leqslant c(4), c^{\prime}(4)<14 .
$$

The bound $c(3) \geqslant 8$ follows from 4.7.3. To show that $c^{\prime}(3)<9$, take an irreducible real curve $A$ of degree 3 with two real components and pick 5 points on its two-sided component and 4 points on the one-sided component. By Poincaré duality, any real curve $A^{\prime}$ of degree 3 through the 9 chosen points has a fifth intersection point with the one-sided component (or, as a special case, one of the four chosen points is multiple). The Bezout theorem implies then that $A^{\prime}$ coincides with $A$, but no component of $A$ contains all the 9 points.

The bound $c(4) \geqslant 13$ is a straightforward consequence of 4.6.7.

To show that $c^{\prime}(4)<14$, take a real curve $A$ of degree 4 with 4 real components and pick 11 points on one of the ovals and one point on each of the other ones. By Poincaré duality, any real curve $A^{\prime}$ of degree 4 through the 14 chosen points intersects each oval of $A$ at least one more point (or has a multiple intersection point). Thus, the total number of intersection points is $>16$, and the Bezout theorem implies that $A^{\prime}$ coincides with $A$ (as a curve of degree $\geqslant 4$ with the maximal number of components, $A$ is nonsingular and irreducible). It remains to notice that none of the component of $A^{\prime}=A$ contains all the 14 points.

Remark. In the above proof of $c_{3}^{\prime}<9$ the 9 points are chosen so that they do not lie on a real cubic other than $A$. From the proof it follows also that a connected real cubic can be traced through 9 points of an $M$-cubic $C$ if and only if they belong to a cubic $C^{\prime}$ other than $C$. The existence of $C^{\prime}$ can be expressed in terms of the group structure on $C$ : it exists if and only if the sum of the 9 points equals 0 (or, more precisely, 9 times an inflection point). Certainly, the set of 9 points should be invariant with respect to the complex conjugation; then $C^{\prime}$ can be chosen real. As a consequence, the 9-tuples not on a connected cubic form an open nonempty subset in the space of all 9-tuples of points of $\mathbb{R} \mathbb{P}^{2}$.

The precise values of the other terms of $\{c(q)\},\left\{c^{\prime}(q)\right\}$ are not known. Below we give a few simple general lower and upper bounds. 
4.7.2. Proposition. For any $q$ one has $c(q) \geqslant 3 q-1$.

Digression: real rational curves. As far as we know, the following problem is still open: is it possible to trace an irreducible real rational curve (or, more precisely, its connected component) of degree $q$ through any set of $3 q-1$ real points in general position. In [R3] the question is answered in the affirmative; however, the proof has been never published; probably, it contained a gap. Alternatively, one can lift the general position assumption and pose the question of existence of some (not necessarily irreducible) rational curve.

The first nontrivial case (and the only one where the complete answer is known) is $q=3$ : through 8 generic points one can trace 12 cubics; depending on the position of the points, 8,10 , or 12 of them are real. (All the three values occur; all the 12 rational cubics in a pencil are real if and only if the pencil contains two cubics with a solitary double point.)

There also are a few simple results on collections of points with prescribed multiplicities. For example, if $q-1$ of the $3 q-1$ points coincide, the answer is in the affirmative. In fact, in an affine coordinate system such that the multiple point, $M$, and one of the other $2 q$ points, $N$, lie at infinity, the problem becomes linear: if the $x$-axis contains $N$ and the $y$-axis contains $M$, it reduces to interpolating $2 q-1$ values $y_{j}$ by a rational function $y=P(x) / Q(x)$ of multidegree $(\operatorname{deg} P, \operatorname{deg} Q)=(q-1, q-1)$. The explicit solution is called Cauchy interpolation formula. As this construction shows, through any $2 q$ real points one can trace a real rational curve of degree $q$. The result can also be obtained by a proper small perturbation of a family of $q$ lines through the given points, considering in the space $\mathbb{R} C_{q}$ of real curves of degree $q$ the subvariety of curves having $\frac{1}{2}(q-1)(q-2)$ double points and passing through the given points. Replacing lines by conics or rational cubics yields slightly better results: a real rational curve of degree $q$ can be traced through any $\frac{5}{2} q$ or, respectively, $\frac{8}{3} q$ real points.

4.7.3. Proposition. Given 8 generic points in $\mathbb{R P}^{2}$, denote by $c_{+}$and $c_{-}$the numbers of real cubics through them with, respectively, a solitary double point and a real crossing point. Then $c_{-}-c_{+}=8$ and $c_{-}+c_{+} \leqslant 12$. In particular, there are at least 8 real rational cubics through the given points.

Proof. The pencil of cubics through the 8 given points defines a (singular) fibration $X \rightarrow \mathbb{R} \mathbb{P}^{1}$, where $X$ is $\mathbb{R} \mathbb{P}^{2}$ blown up at the 8 points, and the statement follows from counting the Euler characteristic of $X$.

Note that in a similar way one can prove that in any pencil of cubics there is one with connected real part.

Proof of 4.7.2. In view of 4.7.1 it suffices to consider $q>4$. The proof depends on the residue $q \bmod 4$.

If $q=4 k+1, k \in \mathbb{Z}$, then $3(4 k+1)-1=12 k+2$. Trace a real line through any two of the $12 k+2$ points. The other $12 k$ points decompose into $k$ groups of 12 points. Each group and one of the first 2 points can be joined by a real curve of degree 4 with connected real point set. The product of the line and the constructed curves of degree 4 is of degree $4 k+1$; its real point set is connected and contains all the $12 k+2$ points.

If $q=4 k+2, k \in \mathbb{Z}$, then $3(4 k+2)-1=12 k+5$. Trace a real conic through any five of the $12 k+5$ points. The other $12 k$ points decompose into $k$ groups 
of 12 points. Each group and one of the first 5 points can be joined by a real curve of degree 4 with connected real point set. The product of the conic and the constructed curves of degree 4 is of degree $4 k+2$; its real point set is connected and contains all the $12 k+5$ points.

If $q=4 k+3, k \in \mathbb{Z}$, then $3(4 k+3)-1=12 k+8$, and a curve is constructed as above, starting with a cubic through any eight of the $12 k+8$ points.

If $q=4 k, k \in \mathbb{Z}$, then the bound $12 k-1$ can be replaced with $12 k+1$. Pick one of the points. The other $12 k$ points decompose into $k$ groups of 12 points. Each group and the fixed point can be joined by a real curve of degree 4 with connected real point set. The product of these curves has degree $4 k$, its real point set is connected and contains all the $12 k+1$ points.

4.7.4. Proposition. If $q$ is odd, then $c^{\prime}(q) \leqslant \frac{1}{2} q(q+3)-\left[\frac{1}{8}\left(q^{2}-1\right)\right]$. If $q$ is even, then $c^{\prime}(q) \leqslant \frac{1}{2} q(q+3)-\left[\frac{1}{8} q(q-2)\right]$.

Proof. First, consider the case of $q$ odd, $q \geqslant 3$. (For $q=1$ the inequality follows from $\left.c^{\prime}(1)=2\right)$. Put $q=2 k-1$ and $m=k+1$. Then the bound takes the form $c^{\prime}(q) \leqslant \frac{3}{2} k(k+1)-1$. Take an $M$-curve $A$ of degree $m$. Its real part has $\frac{1}{2} k(k-1)+1$ connected components. Pick $3 k$ points on one of the components and 3 points on each of the others, totally $\frac{3}{2} k(k+1)$ points. If $k$ is even, the distinguished component should be one-sided. Suppose that $c^{\prime}(q) \geqslant \frac{3}{2} k(k+1)$. Then there is a real curve $B$ of degree $q$ with a connected component which contains all the $\frac{3}{2} k(k+1)$ points. By the Poincaré duality, each of the components of $\mathbb{R} A$ intersects $\mathbb{R} B$ in at least one additional point (or one of the intersection points is multiple). Thus, the number of intersection points of $A$ and $B$, counted with their multiplicity, is at least $2 k^{2}+k+1>m q$, and, by the Bezout theorem, $B$ contains $A$ as a component (as an $M$-curve, $A$ is irreducible). Thus, $B$ decomposes into two curves, $A$ and $B^{\prime}$, of degree $m=k+1$ and $q-m=k-2$, respectively. Furthermore, $\mathbb{R} A$ is contained in a connected component of $\mathbb{R} B^{\prime}$; hence, $A$ and $B^{\prime}$ intersect in at least $k(k-1)>(k+1)(k-2)$ points. Since $A$ is irreducible, this contradicts to the Bezout theorem.

If $q$ is even put $q=2 k$ and $m=q+4=2(k+2)$ and consider a Wiman $M$-curve $A$ of degree $m$ (i.e., a curve with the real scheme $\langle(k+1)(k+3)+1\rangle \sqcup \frac{1}{2} k(k+1)\langle 1\langle 1\rangle\rangle$; it can be constructed by a small perturbation of the square of an $M$-curve of degree $k+2)$. Suppose that $c^{\prime}(q) \geqslant \frac{1}{2} q(q+3)-\frac{1}{8} q(q-2)+1=\frac{3}{2} k(k+1)+2 k+1$. Pick one point in the inner oval of each nest of $A$ and one point in $k^{2}+3 k+1$ of $k^{2}+4 k+4$ empty outer ovals, totally $\frac{3}{2} k(k+1)+2 k+1$ points, and trace through them a connected component of a real curve $B$ of degree $q$. Then $A$ and $B$ intersect in at least $4 k^{2}+8 k+2>4 k^{2}+8 k$ points, which contradicts to the Bezout theorem.

Remark. The case of $q$ odd can be treated in an alternative way, similar to that of $q$ even. Surprisingly, this does not improve the bound.

4.7.5. Corollary. One has $14 \leqslant c(5) \leqslant c^{\prime}(5) \leqslant 17,17 \leqslant c(6) \leqslant c^{\prime}(6) \leqslant 24$, and $20 \leqslant c(7) \leqslant c^{\prime}(7) \leqslant 29$.

The upper bound for $c^{\prime}(6)$ can easily be improved:

4.7.6. Proposition. $c^{\prime}(6) \leqslant 23$.

Proof. Suppose that $c^{\prime}(6) \geqslant 24$, pick a (necessarily irreducible) $M$-curve of degree 9 with the scheme $J \sqcup\langle 1\langle a\rangle\rangle \sqcup\langle 1\langle b\rangle\rangle \sqcup\langle 1\langle c\rangle\rangle \sqcup\langle 1\langle d\rangle\rangle, a, b, c, d>0, a+b+c+d=24$, 

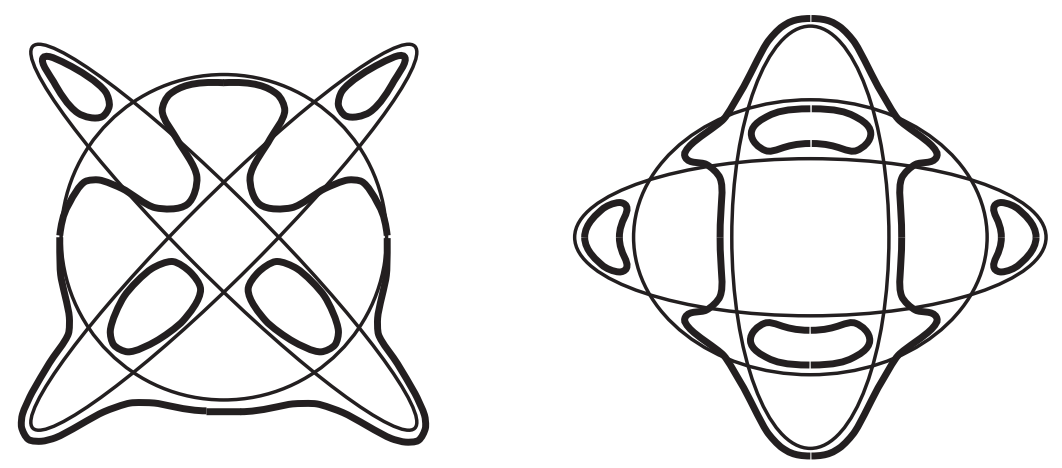

Figure 5. Sextic without and with a lock

and trace a connected component of a real sextic through all the 24 empty ovals. The two curves intersect in at least $48+8=56>54$ points.

4.8. Obstructions to rigid isotopy. The topological characteristics of a real curve, such as its real scheme, type, and complex scheme (i.e., real scheme enhanced with the complex orientations) for curves of type I are preserved under rigid isotopies. Another powerful source of obstructions is the Bezout theorem applied to appropriate auxiliary curves.

To our knowledge, obstructions derived from the Bezout theorem have never been studied systematically. Thus, we confine ourselves to an explanation of the principal idea and its simplest realizations taken from [Fi2] and [Ma2].

Let us start from auxiliary curves of the lowest possible degree: consider lines. In this case we look for pairs of points, in $\mathbb{R P}^{2} \backslash \mathbb{R} A$ for simplicity, selected topologically in such a way that the line $L$ through them would intersect the curve only at real points. If such a selection is possible, the position of $\mathbb{R} A$ with respect to $\mathbb{R} L$ is preserved under rigid isotopies of $A$. In particular, the position of $\mathbb{R} A$ is the same with respect to all real lines close to $L$.

To make this idea more constructive, let us introduce the following definition. A real line $L$ with 2 marked points $p_{1}, p_{2} \in \mathbb{R} L$ outside the real part of a real nonsingular curve $A$, is called a lock of $A$, if (1) the number of points in $S=\mathbb{R} L \cap \mathbb{R} A$ is equal to the degree of $A,(2)$ for any pair of points (possibly coinciding) in $S$ bounding a segment in $\mathbb{R} L \backslash\left(\left\{p_{1}, p_{2}\right\} \cup S\right)$ and an arc in $\mathbb{R} A \backslash S$, the cycle formed by the segment and the arc either is not homologous to 0 in $\mathbb{R P}^{2}$ or envelops at least one oval of $A$. It is clear that a lock extends continuously to any rigid isotopy of $A$ and, moreover, regardless of the extension the topological type of the quadruple $\left(\mathbb{R P}^{2}, \mathbb{R} A \cup \mathbb{R} L, \mathbb{R} L,\left\{p_{1}, p_{2}\right\}\right)$ does not change. Even this simple consideration gives us two invariants: existence of a lock and the topological types of the quadruples corresponding to all locks.

As an example, consider two curves of degree 6 with the scheme $\langle 2\rangle \sqcup\langle 1\langle 2\rangle\rangle$ constructed from 2 products of ellipses, as shown in Figure 5. The first curve has no lock. For the second one, any pair of points, one inside each of the two outer ovals, define a lock. Hence, the curves are not rigidly isotopic.

There are situations where simultaneous consideration of several locks is useful. Example: the two curves of degree 5 with 4 ovals shown in Figure 6 . In both the curves any pair of ovals defines a lock; hence, any triple defines a triple lock. The 

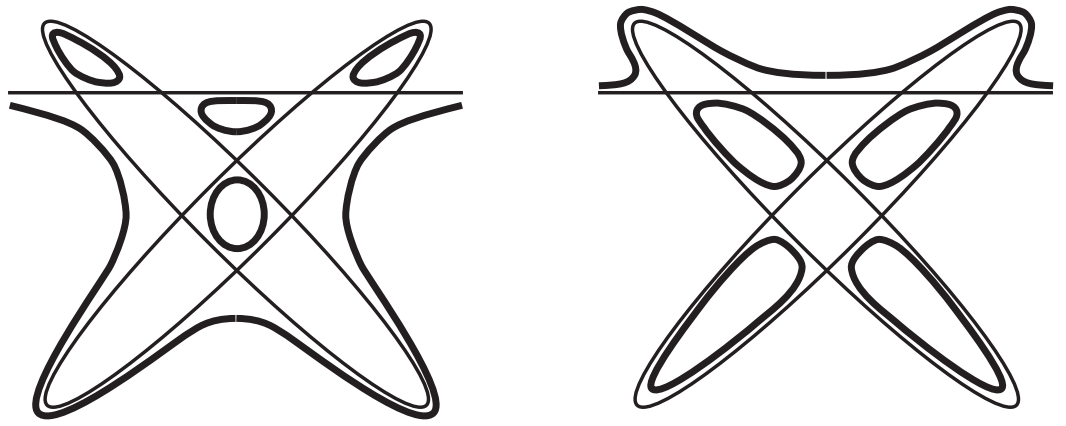

Figure 6 . Two quintic with different triple locks
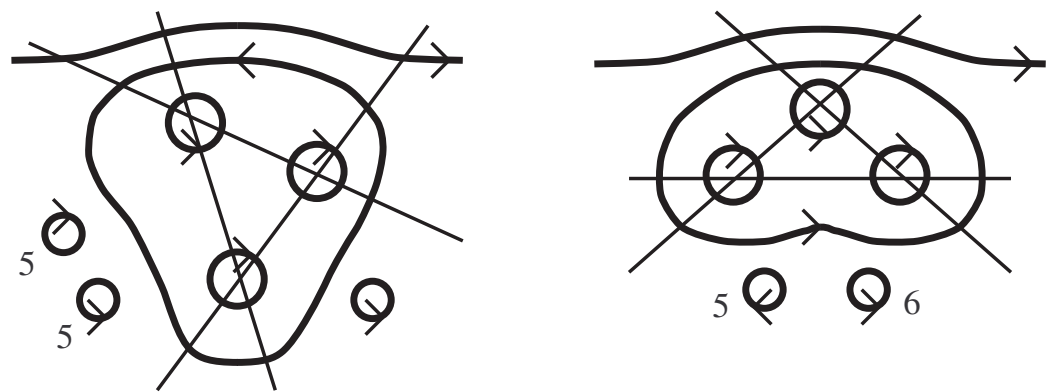

Figure 7. Two curves of degree seven with different triple locks

first curve has a triple of ovals whose triple lock has the following properties: one of the connected components of the complement of the three lines contains the fourth oval and does not intersect the one-sided component. The other curve has no such triple. Hence, the two curves are not rigidly isotopic.

In fact, in both the examples the curves differ by their complex scheme: as one can see from the construction, in each example one curve is of type I and the other, of type II. Pairs of not rigidly isotopic curves with the same complex scheme appear first in degree 7. An example, due to A. Marin [Ma2], is shown in Figure 7. The two curves with the complex scheme $\left\langle J \sqcup 1^{+}\left\langle 1^{+} \sqcup 2^{-}\right\rangle \sqcup 6^{+} \sqcup 5^{-}\right\rangle$are constructed by the Hilbert method, with a difference at the last step. The three inner ovals define a triple lock, and the two curves differ by the distribution of the outer ovals among the connected components of the complement of the three lines.

Another example of not rigidly isotopic curves of degree 7 with the same complex scheme was constructed by T. Fiedler [Fi2]. (In his example the curves are not dividing.)

There are examples where quadrics are used for locks instead of lines. In one of them, due to I. Itenberg, the inner ovals of the two negative injective pairs of the sextic $\left\langle 1\left\langle 3^{+} \sqcup 2^{-}\right\rangle\right\rangle \sqcup\langle 5\rangle$ are separated from each other by a quadric trough the three other inner ovals and two outer ovals. Certainly, locks of higher degrees can also be considered. We interrupt this theme because it is not yet sufficiently understood.

Back to Marin's example, note that it is not known whether the two curves are equivariantly isotopic. More generally, the following two fundamental problems are 
still open: Are there Bezout like obstructions which are not flexible? Do there exist curves which are equivariantly isotopic but not rigidly isotopic? (When this survey was ready, S. Fiedler-Le Touzé and S. Orevkov announced that they found a flexible affine sextic not realizable algebraically because of a Bezout like obstructions in respect to cubics.)

4.9. Curves on Quadrics. We conclude this section with a brief account of known results on curves on nonsingular quadrics, which seem to be the only case (except $\mathbb{P}^{2}$ ) that has been seriously studied. Let $X$ be such a quadric. From the complex point of view $X=\mathbb{P}^{1} \times \mathbb{P}^{1}$, and up to biholomorphism $X$ admits two anti-holomorphic involutions with nonempty real part; the resulting real surfaces are the hyperboloid, which is an $M$-surface with real part homeomorphic to torus, and the ellipsoid, an $(M-1)$-surface with $\mathbb{R} X \cong S^{2}$.

Fix a pair $P_{1}, P_{2}$ of generatrices of $X$. The fundamental classes $\left[P_{1}\right],\left[P_{2}\right]$ form a basis of $H_{2}(X) \cong \mathbb{Z} \oplus \mathbb{Z}$; the intersection form is given by $\left[P_{1}\right]^{2}=\left[P_{2}\right]^{2}=0$, $\left[P_{1}\right] \circ\left[P_{2}\right]=1$. For any other curve $A \subset X$ one has $[A]=m_{1}\left[P_{1}\right]+m_{2}\left[P_{2}\right]$ for some nonnegative integers $m_{1}, m_{2}$. The pair $\left(m_{1}, m_{2}\right)$ is called the bidegree of $A$. If $\left(x_{0}: x_{1}\right),\left(y_{0}: y_{1}\right)$ are homogeneous coordinates in $P_{1}, P_{2}$ respectively, then $A$ is given by a polynomial

$$
F\left(x_{0}, x_{1} ; y_{0}, y_{1}\right)=\sum_{i, j=1}^{m_{1}, m_{2}} a_{i j} x_{1}^{i} x_{0}^{m_{1}-i} y_{1}^{j} y_{0}^{m_{2}-j}
$$

homogeneous of degree $m_{1}$ in $\left(x_{0}, x_{1}\right)$ and of degree $m_{2}$ in $\left(y_{0}, y_{1}\right)$. Such curves form a projective space of dimension $m_{1} m_{2}+m_{1}+m_{2}$; if $A$ is nonsingular, the genus of $A$ equals $\left(m_{1}-1\right)\left(m_{2}-1\right)$ (see $(4.9) ; K_{X}$ has bidegree $(-2,-2)$ ).

In the case of hyperboloid conj acts by conjugating all the four coordinates; hence, $A$ is real if and only if all $a_{i j}$ are real. In the case of ellipsoid conj acts via $\left[\left(x_{0}: x_{1}\right),\left(y_{0}: y_{1}\right)\right] \mapsto\left[\left(\bar{y}_{0}: \bar{y}_{1}\right),\left(\bar{x}_{0}: \bar{x}_{1}\right)\right]$, and $A$ is real if and only if $a_{i j}=\bar{a}_{j i}$ (in particular, $\left.m_{1}=m_{2}\right)$.

From now on we assume that $A$ is a real curve. First, let $(X$, conj) be a hyperboloid. The fundamental classes $\left[\mathbb{R} P_{1}\right],\left[\mathbb{R} P_{2}\right]$, endowed with some orientations (which are to be fixed) form a basis of $H_{1}(\mathbb{R} X) \cong \mathbb{Z} \oplus \mathbb{Z}$. The real part $\mathbb{R} A$ may have components of two types: contractible in $\mathbb{R} X$ and noncontractible. The contractible components are called ovals, their number is denoted by $l$. Each oval bounds a disk in $\mathbb{R} X$, which is called the interior of this oval. All the noncontractible components realize the same nontrivial class $\left(c_{1}, c_{2}\right) \in H_{1}(\mathbb{R} X)$, where $c_{1}, c_{2}$ must be relatively prime. The number of such components is denoted by $h$. In order to encode the isotopy type of $\mathbb{R} A \subset \mathbb{R} X$ we will use the notation

$$
\left\langle\left(c_{1}, c_{2}\right), \text { scheme }_{1},\left(c_{1}, c_{2}\right), \text { scheme }_{2}, \ldots,\left(c_{1}, c_{2}\right), \text { scheme }_{h}\right\rangle,
$$

where scheme $1, \ldots$, scheme $_{h}$ are the schemes of ovals of $\mathbb{R} A$ in the annulus bounded by two consequent noncontractible components.

If both $m_{1}$ and $m_{2}$ are even, $\mathbb{R} A$ separates $\mathbb{R} X$ into two parts $\mathbb{R} X_{ \pm}$. Obviously, $\chi\left(\mathbb{R} X_{-}\right)=-\chi\left(\mathbb{R} X_{+}\right)$. If all the components of $\mathbb{R} A$ are ovals, we denote by $\mathbb{R} X_{+}$ the part of genus 1 .

4.9.1. Prohibitions for curves on a hyperboloid. Let $A$ be a real curve of bidegree $\left(m_{1}, m_{2}\right)$ on a hyperboloid $X$, and $l, h, c_{1}, c_{2}$ as above. Then $A$ satisfies the following restrictions: 


\section{The Harnack inequality:}

(1) $l+h \leqslant\left(m_{1}-1\right)\left(m_{2}-1\right)+1$.

Congruences: Assume that $m_{1}, m_{2}$ are even and $\frac{1}{2}\left(m_{1} c_{2}+m_{2} c_{1}\right)=c_{1} c_{2} \bmod 2$. Then (with the above convention on the choice of $\mathbb{R} X_{+}$)

(2) If $A$ is an $M$-curve, then $\chi\left(\mathbb{R} X_{+}\right)=\frac{1}{2} m_{1} m_{2} \bmod 8$;

(3) If $A$ is an $(M-1)$-curve, then $\chi\left(\mathbb{R} X_{+}\right)=\frac{1}{2} m_{1} m_{2} \pm 1 \bmod 8$;

(4) If $A$ is an $(M-2)$-curve and $\chi\left(\mathbb{R} X_{+}\right)=\frac{1}{2} m_{1} m_{2}+4 \bmod 8$, then $A$ is of type I;

(5) If $A$ is of type I, then $\chi\left(\mathbb{R} X_{+}\right)=0 \bmod 4$.

\section{Consequences of the Bezout Theorem:}

(6) $h c_{i}=m_{1} \bmod 2$ and $h\left|c_{i}\right| \leqslant m_{1}$ for $i=1,2$;

(7) if $\mathbb{R} A$ has $r$ pairwise disjoint nests of depths $s_{1}, \ldots, s_{r}$, then

$$
\sum_{j=1}^{r} s_{j} \leqslant \frac{1}{2}\left(m_{1}-h\left|c_{1}\right|+[r / 2]\left(m_{2}+h\left|c_{2}\right|\right)\right)
$$

(and a similar inequality with $m_{1}, c_{1}$ interchanged with $m_{2}, c_{2}$ ).

Proof. (1) is the classical Harnack-Klein bound. (2)-(5) follow from 3.3.2 and 3.3.4. Statement (6) is the real version of the Bezout theorem (see 4.6) applied to $A$ and curves of bidegree $(1,0)$ and $(0,1)$, and $(7)$ is proved by applying the Bezout theorem to $A$ and the curve of bidegree $([r / 2], 1)$ traced through $r$ points inside the innermost ovals of the nests (see [Zv3]).

Remark. In a weaker form the above congruences appeared in [Mat1], [Mat2]. This inspired [Mik1], where the congruences were improved and generalized to take the form of 3.3.2.

If $(X$, conj $)$ is an ellipsoid, all the components of $\mathbb{R} A$ are contractible and are called ovals; their number is denoted by $l$. Fix an exterior point $\infty \in \mathbb{R} X \backslash \mathbb{R} A$ and define the interior of an oval $C \subset \mathbb{R} X$ as the component of $\mathbb{R} X \backslash C$ which does not contain $\infty$. Then a natural partial order arises, which can be encoded as usual.

Since $\mathbb{R} X \cong S^{2}$, any curve $\mathbb{R} A$ divides $\mathbb{R} X$ into two parts, denoted by $\mathbb{R} X_{ \pm}$.

4.9.2. Prohibitions for curves on an ellipsoid. Let $A$ be a real curve of bidegree $(m, m)$ on an ellipsoid $X$, and $l$ the number of components of $\mathbb{R} A$. Then $A$ satisfies the following restrictions:

The Harnack inequality:

(1) $l \leqslant\left(m_{1}-1\right)\left(m_{2}-1\right)+1$.

Congruneces: Let $m$ be odd.

(2) If $A$ is an $M$-curve, then $\chi\left(\mathbb{R} X_{ \pm}\right)=\frac{1}{2}\left(m^{2}+1\right) \bmod 8$;

(3) If $A$ is an $(M-1)$-curve, then $\chi\left(\mathbb{R} X_{ \pm}\right)=\frac{1}{2}\left(m^{2}+1\right) \pm 1 \bmod 8$;

(4) If $A$ is an $(M-2)$-curve and $\chi\left(\mathbb{R} X_{ \pm}\right)=\frac{1}{2}\left(m^{2}+1\right)+4 \bmod 8$, then $A$ is of type I;

(5) If $A$ is of type I, then $\chi\left(\mathbb{R} X_{ \pm}\right)=1 \bmod 4$. 


\section{Consequences of the Bezout Theorem:}

(6) The total number of ovals in any three pairwise disjoint nests does not exceed $m$.

Proof is similar to that of 4.9.1.

Next two well known statements are found, e.g., in [Zv4]. Their standard proofs, reproduced below, serve as a model for a number of similar cases (such as rational and hyperelliptic curves on ruled surfaces, intersections of quadrics, or homotopy classification of nondegenerate homogeneous polynomial vector fields).

4.9.3. Curves of Bidegree $(m, 1)$. A curve of bidegree $(m, 1)$ on a hyperboloid is determined up to rigid isotopy by its real scheme, which is $\langle(w, 1)\rangle$ with $-m \leqslant$ $w \leqslant m$ and $w=m \bmod 2$. All such curves are of type I.

There are two rigid isotopy classes of curves of bidegree $(1,1)$ on an ellipsoid: $\langle 0\rangle$ and $\langle 1\rangle$, the latter being of type I.

Proof. A nonsingular curve of bidegree $(m, 1)$ on a hyperboloid has equation of the form

$$
y_{0} a\left(x_{0}, x_{1}\right)+y_{1} b\left(x_{0}, x_{1}\right)=0,
$$

where $a$ and $b$ are nonzero homogeneous polynomials of degree $m$ without common roots. The roots of $a$ and $b$ being fixed, the polynomials are recovered uniquely up to constant factors. Any pair of consecutive real root of one of these polynomials not separated by real roots of the other one can be removed to the complex domain. After all such pairs are removed, $|w|$ is the number of real roots of $a$ (or $b$ ); for each nonzero value of $|w|$ there are two classes of curves which differ by the signs of $a$ and $b$ at a fixed generic point.

Any curve of bidegree $(1,1)$ on an ellipsoid is cut by a plane, and it is obvious that there are only two possibilities.

4.9.4. Curves of bidegree $(m, 2)$. A curve of bidegree $(m, 2)$ on a hyperboloid is determined up to rigid isotopy by its real scheme, which is either

$$
\left\langle(0,1), \alpha_{1},(0,1), \alpha_{2}, \ldots,(0,1), \alpha_{h}\right\rangle
$$

with $0 \leqslant h \leqslant m, h=m \bmod 2$, and $h+\sum \alpha_{i} \leqslant m$, or

$$
\left\langle 2\left(\frac{1}{2} w, 1\right)\right\rangle \text { for } m \text { even or }\langle(w, 2)\rangle \text { for } m \text { odd }
$$

with $-m \leqslant w \leqslant m$ and $w=m \bmod 2$. The latter scheme and the $M$-schemes are of type I; the others are of type II.

There are three rigid isotopy classes of curves of bidegree $(2,2)$ on an ellipsoid: $\varnothing,\langle 1\rangle$, and $\langle 2\rangle$. The only scheme of type $\mathrm{I}$ is $\langle 2\rangle$.

The proof given below works, more or less without changes, whenever the curves under consideration are hyperelliptic (so that the hyperelliptic structure is induced from a ruling of the surface). For this reason we reproduce all the essential details.

Proof. A curve of bidegree $(m, 2)$ on a hyperboloid has equation of the form

$$
y_{0}^{2} a\left(x_{0}, x_{1}\right)+2 y_{0} y_{1} b\left(x_{0}, x_{1}\right)+y_{1}^{2} c\left(x_{0}, x_{1}\right)=0,
$$


where $a, b$, and $c$ are homogeneous polynomials of degree $m$. Such a curve is nonsingular if and only if the discriminant $D=b^{2}-a c$ has no multiple roots.

Let $V=\left\{\left(x_{0}: x_{1}\right) \in \mathbb{R P}^{1} \mid D\left(x_{0}, x_{1}\right) \geqslant 0\right\}$. Consider an affine part $x_{1} \neq 0$. The connected components of the set consisting of the nonsingular curves with fixed $V$ and fixed roots $u_{1}, \ldots, u_{m}$ of $a$ (which may be multiple) are determined by the choice of the values $b\left(u_{i}\right)= \pm \sqrt{D\left(u_{i}\right)}, i=1, \ldots, m$, i.e., by the choice of the sign of $b$ at the real roots of $a$ (which belong to $V$ ) and one of the two branches of the square root at each pair of complex conjugate roots. The components can be joined using the following moves:

(1) a pair of consecutive real roots of $a$ at which $b$ has the same sign can be removed to the complex domain; a pair of complex conjugate roots can be moved to any component of $V$;

(2) the sign of $b$ at a real root of $a$ not separated from $\partial V$ by other roots can be changed (by moving this root to $\partial V$ );

(3) the branch of $\sqrt{D}$ at any pair of complex conjugate roots of $a$ can be changed (by moving this pair to a pair of roots of $D$ ).

Thus, if $V=\varnothing$ or $\partial V \neq \varnothing$, the sign of $b$ at each (real or imaginary) root of $a$ can be reversed and the curve is determined up to rigid isotopy by the components of $V$ and the parity of the number of real roots of $a$ in each component: the corresponding component of the curve is an oval if this number is even or of class $(1,0)$ if it is odd. If $V=\mathbb{R} \mathbb{P}^{1}$, then it contains $|w|$ real roots of $a$ so that $b$ has opposite signs at each pair of consecutive roots. This gives the second group of real schemes in 4.9.4.

Consider now the case of ellipsoid. All empty curves are rigidly isotopic (in fact, in any bidegree), as they form a convex set. If the curve is nonempty, we blow up a real point of this curve and blow down the proper inverse images of the two imaginary generatrices through this point. The result is a nonsingular cubic in $\mathbb{R} \mathbb{P}^{2}$, and the inverse transformation is given by a pair of complex conjugate point of this cubic. The two rigid isotopy classes of cubics correspond to the two classes of curves on the ellipsoid.

4.9.5. Curves of bidegree $(3,3)$. A curve of bidegree $(3,3)$ on a hyperboloid or an ellipsoid is determined up to rigid isotopy by its real scheme and, in the case of hyperboloid, its type. On a hyperboloid the real schemes are

$$
\langle(1, \pm 1), \alpha\rangle \text { with } \alpha \leqslant 4, \quad\langle 3(1, \pm 1)\rangle, \quad\langle(3, \pm 1)\rangle, \quad\langle(1, \pm 3)\rangle .
$$

The schemes $\langle(1, \pm 1), 4\rangle,\langle 3(1, \pm 1)\rangle,\langle(3, \pm 1)\rangle$, and $\langle(1, \pm 3)\rangle$ are of type $\mathrm{I}$; the scheme $\langle(1, \pm 1), 2\rangle$ is of indefinite type; the other schemes are of type II. On an ellipsoid the real schemes are

$$
\langle\alpha\rangle \text { with } \alpha \leqslant 5 \text { and }\langle 1\langle 1\langle 1\rangle\rangle\rangle \text {. }
$$

The schemes $\langle 5\rangle$ and $\langle 1\langle 1\langle 1\rangle\rangle\rangle$ are of type I; the others are of type II.

Similar to 3.5.2, the proof of 4.9.5 is based on enumerating the walls in the space of curves; the latter problem is mainly reduced to the classification of plane quartics with a fixed tangent. Details can be found in [DZ].

The remaining results of this section (Propositions 4.9.6-4.9.8) only list the possible schemes for certain bidegrees. All the schemes not on the lists are prohibited 
by 4.9.1 and 4.9.2. The listed schemes have been realized by D. Gudkov, A. Usachev, and E. Shustin (see [G3], [GU], and [GS]). The assertions on the type of the schemes follow mainly from Rokhlin's formula of complex orientations 4.2.2. Note also that the complex orientations are those and only those which do not contradict to Rokhlin's and Orevkov's formulas. Details and realization of the schemes of indefinite type can be found in Zvonilov [Zv3].

4.9.6. Schemes of bidegree $(m, 3)$. A curve of bidegree $(m, 3)$ with $m>3$ on a hyperboloid can only have one of the following real schemes:

(1) $\langle 3(w, 1)\rangle$ with $3|w| \leqslant m$ and $w=m \bmod 2$;

(2) $\langle(w, 3)\rangle$ with $|w| \leqslant m, w=m \bmod 2$, and $w \neq 0 \bmod 3$;

(3) $\langle(m, \pm 1)\rangle$;

(4) $\langle(w, 1), \alpha\rangle$ with $|w| \leqslant m-2, w=m \bmod 2$, and $0 \leqslant \alpha \leqslant 2 m-2$.

Schemes (1)-(3) are of type I; schemes (4) are of type I when $\alpha=2 m-2$ and are of type II when $\alpha$ is odd or zero.

All the schemes above (including complex orientations) were realized by A. Korchagin [Ko], who used Viro's patching method. According to V. Zvonilov, they can as well be realized by $T$-curves. A new promising approach to the study of trigonal curves on ruled surfaces by means of Grothendieck's dessins d'enfant applied to the discriminant of the curve was recently suggested by S. Orevkov [Or2].

4.9.7. Schemes of bidegree $(4,4)$ on a hyperboloid. The following is a complete list of real schemes of bidegree $(4,4)$ on a hyperboloid:

(1) $\langle 4(1,0)\rangle,\langle 4(0,1)\rangle$, and $\langle 4(1, \pm 1)\rangle$;

(2) $\langle 2(2, \pm 1)\rangle$ and $\langle 2(1, \pm 2)\rangle$;

(3) $\langle(1, \pm 1), \alpha,(1, \pm 1), \beta\rangle$ with $0 \leqslant \alpha+\beta \leqslant 8$ and $\alpha \geqslant \beta$;

(4) $\langle(1,0), \alpha,(1,0), \beta\rangle$ and $\langle(0,1), \alpha,(0,1), \beta\rangle$ with either $0 \leqslant \alpha+\beta \leqslant 6$ and $\alpha \geqslant \beta$, or $(\alpha, \beta)=(8,0),(4,4),(7,0)$, or $(4,3)$;

(5) $\langle\alpha \sqcup 1\langle\beta\rangle\rangle$ with either $0 \leqslant \alpha+\beta \leqslant 7$, or $(\alpha, \beta)=(0,9),(4,5),(8,1),(0,8)$, $(3,5),(4,4),(7,1)$, or $(8,0)$;

(6) $\langle 2\langle 1\rangle\rangle$;

(7) $\langle 0\rangle$.

Schemes (1), (2), and (6) are of type I. Scheme (7) is of type II. Schemes (3) are of type I if $\alpha+\beta=8$, are of type II if $\alpha+\beta$ is odd or $(\alpha, \beta)=(0,0),(1,1)$, or $(4,0)$, and are of indefinite type otherwise. Schemes (4) are of type I if $(\alpha, \beta)=(8,0)$, $(4,4)$, or $(5,1)$, are of indefinite type if $(\alpha, \beta)=(3,3),(4,0),(2,2)$, or $(1,1)$, and are of type II otherwise. Schemes (5) are of type I if $(\alpha, \beta)=(0,9),(4,5),(8,1)$, $(1,6)$, or $(5,2)$, are of indefinite type if $(\alpha, \beta)=(3,4),(7,0),(2,3)$, or $(4,1)$, and are of type II otherwise.

4.9.8. Schemes of bidegree $(4,4)$ on an ellipsoid. The following is a complete list of real schemes of bidegree $(4,4)$ on an ellipsoid:

(1) $\langle\alpha \sqcup 1\langle\beta\rangle\rangle$ with $0 \leqslant \alpha+\beta \leqslant 9$ and $\alpha \geqslant \beta$;

(2) $\langle 1\langle 1\langle 1\langle 1\rangle\rangle\rangle\rangle$

(3) $\langle 0\rangle$.

Schemes (2) and (1) with $\alpha+\beta=9$ are of type I; schemes (3) and (1) with $\alpha+\beta \leqslant 4$ or even or $(\alpha, \beta)=(5,0)$ are of type II; the other schemes are of indefinite type.

The double covering of a quadric ramified in a curve of bidegree $(4,4)$ is a $K 3$ surface whose Picard group contains $U(2)$ (i.e., a pair $a, b$ with $a^{2}=b^{2}=0$ 
and $a b=2)$, and, vice versa, any $K 3$-surface $X$ with a distinguished subgroup $U(2) \subset \operatorname{Pic} X$ is obtained in this way (see, e.g., [Nik1]). Thus, the curves of bidegree $(4,4)$ can be studied similar to plane sextics. In particular, this techniques can provide their rigid isotopy classification. First steps in this direction are made in [Mat3]. In the case of hyperboloid it will not be a surprise if the list of 4.9.7 turns out to coincide with the classification up to rigid isotopies and automorphisms of the surface. (In the case of ellipsoid there probably are additional invariants.) Note that some curves on the hyperboloid, e.g., $\langle 1\langle 9\rangle\rangle,\langle 4 \sqcup 1\langle 5\rangle\rangle,\langle 1\langle 8\rangle\rangle,\langle 3 \sqcup 1\langle 5\rangle\rangle$, and $\langle 4 \sqcup 1\langle 4\rangle\rangle$, are not chiral, see [Kh7].

As to curves on surfaces of higher degree little is known. We can only cite [Seg] and [Mik4], which treat, respectively, real lines and real quadric sections on real cubic surfaces.

\section{Appendix A. Topology of involutions}

A.1. Smith theory. Most results cited in this section are due to P. A. Smith; proofs can be found, e.g., in [Br, Chapter 3]. Throughout the section we consider a topological space $X$ with involution $c: X \rightarrow X$ and denote $F=$ Fix $c$ and $X^{\prime}=X / c$.

A.1.1. Theorem. There are two natural, in respect to equivariant maps, exact sequences, called (homology and cohomology) Smith sequences of $(X, c)$ :

$$
\begin{aligned}
& \rightarrow H_{p+1}\left(X^{\prime}, F\right) \stackrel{\Delta}{\longrightarrow} H_{p}\left(X^{\prime}, F\right) \oplus H_{p}(F) \stackrel{\mathrm{tr}^{*}+\mathrm{in}_{*}}{\longrightarrow} H_{p}(X) \stackrel{\mathrm{pr}_{*}}{\longrightarrow} H_{p}\left(X^{\prime}, F\right) \rightarrow, \\
& \rightarrow H^{p}\left(X^{\prime}, F\right) \stackrel{\mathrm{pr}^{*}}{\longrightarrow} H^{p}(X) \stackrel{\operatorname{tr}_{*} \oplus \mathrm{in}^{*}}{\longrightarrow} H^{p}\left(X^{\prime}, F\right) \oplus H^{p}(F) \stackrel{\Delta}{\longrightarrow} H^{p+1}\left(X^{\prime}, F\right) \rightarrow .
\end{aligned}
$$

The homology and cohomology connecting homomorphisms $\Delta$ are given by

$$
x \mapsto x \cap \omega \oplus \partial x \quad \text { and } \quad x \oplus f \mapsto x \cup \omega+\delta f,
$$

respectively, where $\omega \in H^{1}\left(X^{\prime} \backslash F\right)$ is the characteristic class of the double covering $X \backslash F \rightarrow X^{\prime} \backslash F$. The images of $\mathrm{tr}^{*}+\mathrm{in}_{*}$ and $\mathrm{pr}^{*}$ consist of invariant classes: $\operatorname{Im} \operatorname{tr}^{*} \subset \operatorname{Ker}\left(1+c_{*}\right)$ and $\operatorname{Im} \operatorname{pr}^{*} \subset \operatorname{Ker}\left(1+c^{*}\right)$.

A.1.2. The transfer homomorphisms $\operatorname{tr}^{*}$ and $\operatorname{tr}_{*}$ in A.1.1 can be described as follows. Represent $X$ as the $S^{0}$-bundle associated with a relative $D^{1}$-bundle $T \rightarrow X^{\prime}$ whose characteristic class is $\omega$. Namely, take the cylinder of the projection $X \rightarrow X^{\prime}$ and for each $f \in F$ contract the fiber over $f$. Then $\operatorname{tr}^{*}$ and $\operatorname{tr}_{*}$ are the compositions

$$
\begin{array}{ll}
\operatorname{tr}^{*}: & H_{p}\left(X^{\prime}, F\right) \stackrel{\mathrm{th}^{-1}}{\longrightarrow} H_{p+1}(T, X) \stackrel{\partial}{\longrightarrow} H_{p}(X), \\
\operatorname{tr}_{*}: & H^{p}(X) \stackrel{\delta}{\longrightarrow} H^{p+1}(T, X) \stackrel{\mathrm{th}^{-1}}{\longrightarrow} H^{p}\left(X^{\prime}, F\right),
\end{array}
$$

where th stands for the Thom isomorphisms.

The following immediate consequences of A.1.1, which we state in the homology setting, has an obvious counterpart for cohomology.

A.1.3. Corollary (of A.1.1).

(1) $\operatorname{dim} H_{*}(F)+2 \sum_{p} \operatorname{dim} \operatorname{Coker}\left(\operatorname{tr}^{p}+\operatorname{in}_{p}\right)=\operatorname{dim} H_{*}(X)$ (Smith identity);

(2) $\operatorname{dim} H_{*}(F) \leqslant \operatorname{dim} H_{*}(X)$ (Smith inequality);

(3) $\operatorname{dim} H_{*}(F) \leqslant \operatorname{dim} H^{1}\left(\mathbb{Z}_{2} ; H_{*}(X)\right)$ (Borel-Swan inequality);

(4) $\operatorname{dim} H_{*}(F)=\operatorname{dim} H_{*}(X) \bmod 2$;

(5) $\chi(X)=\chi(F)+2 \chi\left(X^{\prime}, F\right)$;

(6) $\chi(X)=2 \chi\left(X^{\prime}\right)-\chi(F)$ 
(Recall that $H^{1}\left(\mathbb{Z}_{2} ; H_{*}(X)\right)=\operatorname{Ker}\left(1+c_{*}\right) / \operatorname{Im}\left(1+c_{*}\right)$.)

A.1.4. If A.1.3(2) turns into an equality, i.e., $\operatorname{dim} H_{*}(F)=\operatorname{dim} H_{*}(X)$, one says that $c$ is an $M$-involution. If A.1.3(3) turns into an equality (which is equivalent to $\left.\operatorname{Im}\left(\operatorname{tr}^{*}+\operatorname{in}_{*}\right) \supset \operatorname{Ker}\left(1+c_{*}\right)\right), c$ is called $\left(\mathbb{Z}_{2^{-}}\right)$Galois maximal. (This terminology is introduced by V. A. Krasnov [Kr1]. R. Thom [Th1] calls a dimension $p \in \mathbb{N}$ regular for $(X, c)$ if $\operatorname{Im}\left(\operatorname{tr}^{p}+\operatorname{in}_{p}\right) \supset \operatorname{Ker}\left(1+c_{p}\right)$.)

In general, due to A.1.3(2) and (4), one has $\operatorname{dim} H_{*}(F)=\operatorname{dim} H_{*}(X)-2 d$ for some integer $d \geqslant 0$; in this case $c$ is called an $(M-d)$-involution.

A.1.5. Geometrical construction of the Smith sequences. Introduce the Smith chain complexes

$$
\begin{aligned}
\operatorname{Sm}_{*}(X) & =\operatorname{Ker}\left[\left(1+c_{*}\right): S_{*}(X) \rightarrow S_{*}(X)\right], \\
\operatorname{Sm}_{*}(X, F) & =\operatorname{Ker}\left[\left(1+c_{*}\right): S_{*}(X, F) \rightarrow S_{*}(X, F)\right] .
\end{aligned}
$$

and Smith homology $H_{r}\left(\operatorname{Sm}_{*}(X)\right)$ and $H_{r}\left(\operatorname{Sm}_{*}(X, F)\right)$. There is a canonical isomorphism $\operatorname{Sm}_{*}(X, F)=\operatorname{Im}\left[\left(1+c_{*}\right): S_{*}(X) \rightarrow S_{*}(X)\right]$ and a canonical splitting $\mathrm{Sm}_{*}(X)=S_{*}(F) \oplus \operatorname{Im}\left(1+c_{*}\right)$. Furthermore, tr* $: S_{*}\left(X^{\prime}, F\right) \rightarrow \operatorname{Sm}_{*}(X, F)$ is an isomorphism, and in view of the above identifications the Smith sequences are the long homology and cohomology exact sequences associated with the short exact sequence of complexes

$$
0 \rightarrow \mathrm{Sm}_{*}(X) \stackrel{\text { inclusion }}{\longrightarrow} S_{*}(X) \stackrel{1+c_{*}}{\longrightarrow} \mathrm{Sm}_{*}(X, F) \rightarrow 0 .
$$

A.1.6. Steenrod squares in the Smith sequences. For any $x \in H_{p}\left(X^{\prime}, F\right)$ and $y \in H^{p}(X)$ one has $\operatorname{Sq}^{*} x=\operatorname{tr}^{*} \operatorname{Sq}(x \cap(1+\omega))$ and $\operatorname{tr}_{*} \operatorname{Sq} y=\left(\operatorname{Sqtr}_{*} y\right) \cup(1+\omega)$.

Proof. The statement follows from A.1.2 and the Thom-Cartan formula for sphere bundles (see [Th2]): since the total characteristic class of the relative $D^{1}$-bundle $T \rightarrow X^{\prime}$ is $1+\omega$, one has $\operatorname{th}^{-1} \mathrm{Sq}(x \cap(1+\omega))=\mathrm{Sq}^{-1} x$ and $\operatorname{th}^{-1} \mathrm{Sq} y^{\prime}=$ $\left(\mathrm{Sq} \mathrm{th}^{-1} y^{\prime}\right) \cup(1+\omega)$, where $y^{\prime}=\delta y$.

A.1.7. Corollary. For any $x \in H_{p}\left(X^{\prime}, F\right)$ and $y \in H^{p}(X)$ one has $\mathrm{Sq}_{1} \operatorname{tr}^{*} x=$ $\operatorname{tr}^{*} \mathrm{Sq}_{1} x+\mathrm{in}_{*} \partial x$ and $\operatorname{tr}_{*} \mathrm{Sq}^{1} y=\mathrm{Sq}_{1} \operatorname{tr}_{*} y+\delta \operatorname{in}^{*} y$.

Proof. Since the Smith sequences are exact, $\operatorname{tr}^{*}(x \cap \omega)=\operatorname{in}_{*} \partial x$ and $\left(\operatorname{tr}_{*} y\right) \cup \omega=$ $\delta \operatorname{in}^{*} y$. Hence, the statement follows from A.1.6.

A.1.8. Relative version of the Smith sequences. If $A \subset X$ is a $G$-invariant subspace, there is a natural exact sequence

$$
\begin{aligned}
\rightarrow H_{p+1}\left(X^{\prime}, F \cup A^{\prime}\right) \stackrel{\Delta}{\longrightarrow} H_{p}\left(X^{\prime}, F \cup A^{\prime}\right) \oplus H_{p}\left(F, F \cap A^{\prime}\right) \stackrel{\operatorname{tr}^{*}+\mathrm{in}_{*}}{\longrightarrow} \\
\stackrel{\operatorname{tr}^{*}+\mathrm{in}_{*}}{\longrightarrow} H_{p}(X, A) \stackrel{\mathrm{pr}_{*}}{\longrightarrow} H_{p}\left(X^{\prime}, F \cup A^{\prime}\right) \rightarrow
\end{aligned}
$$

(and a similar sequence in cohomology), where $A^{\prime}=A / c$. Its properties and consequences are similar to those of the absolute Smith sequence. In particular, the Smith inequality A.1.3(2) turns into

$$
\operatorname{dim} H_{*}(F, F \cap A) \leqslant \operatorname{dim} H_{*}(X, A) .
$$


A.2. Kalinin's spectral sequence. In this section we describe a relatively new powerful tool, so called Kalinin's spectral sequence. Originally it was introduced by I. Kalinin $[\mathrm{Ka}]$ as a stabilized version of the Borel-Serre spectral sequence of the fibration $X \times_{\mathbb{Z}_{2}} S^{\infty} \rightarrow \mathbb{R} \mathbb{P}^{\infty}$, where $X$ is a topological space with involution $c$ and $S^{\infty}$ is considered with the standard antipodal involution. The sequence starts at the homology $H_{*}(X)$ and converges to the total homology $H_{*}($ Fix $c)$. The resulting filtration $\mathcal{F}^{*}$ on $H_{*}(\mathrm{Fix} c)$ and the isomorphisms $\mathrm{bv}_{p}$ between the limit term of the spectral sequence and $\operatorname{Gr}_{\mathcal{F}} H_{*}$ (Fix $c$ ) were discovered by O. Viro geometrically before Kalinin's work and were primarily related to the Smith exact sequence. The relation between the two construction is established in [Deg1]. As is shown in [DK1] (preprint version) or [DIK], Kalinin's spectral sequence can be derived from the Smith exact sequence as well (see A.2.4).

A construction similar to Kalinin's spectral sequence was studied by V. A. Krasnov in a series of papers; see, e.g., [Kr2].

Below we give an alternative, geometrical, description of the homology version of Kalinin's spectral sequence and Viro homomorphisms $b_{*}$ and state their main properties. (As usual, the cohomology version is obtained by raising all indices and reversing all arrows; we mention it when speaking about multiplications and Poincaré duality.) Proofs of these results and/or further references can be found in [DK1] and [DIK]. The differentials ${ }^{r} d_{p}$ of the spectral sequence and Viro homomorphisms $\mathrm{bv}_{p}$ are often regarded as additive relations (i.e., partial multivalued homomorphisms) $H_{p}(X) \rightarrow H_{p+r-1}(X)$ and bv ${ }_{p}: H_{*}($ Fix $c) \rightarrow H_{p}(X)$, respectively. As our approach is geometrical, we have to use chains; depending on the nature of $X$ one may regard them as singular, simplicial, smooth, etc. To assure convergence of the spectral sequence, $X$ must satisfy certain conditions, which depend on the homology theory chosen (e.g., sheaf theories and locally compact finite dimensional spaces). Certainly, these conditions are always fulfilled for compact smooth manifolds and finite $C W$-complexes.

Thus, let us fix a good (see above) topological space $X$ with involution $c$. Consider the partial homomorphisms bv ${ }_{p}: H_{*}($ Fix $c) \rightarrow H_{p}(X)$ and the $\mathbb{Z}$-graded spectral sequence $\left({ }^{r} E_{*}(X),{ }^{r} d_{*}\right)$ defined as follows:

(1) $\mathrm{bv}_{0}$ is zero on $H_{\geqslant 1}(\mathrm{Fix} c)$ and its restriction to $H_{0}(\mathrm{Fix} c)$ coincides with the inclusion homomorphism;

(2) $\mathrm{bv}_{p}$ is defined on a (nonhomogeneous) element $x \in H_{*}($ Fix $c)$ represented by a cycle $\sum x_{i}$ (where $x_{i}$ is the $i$-dimensional component of $x$ ) if and only if there exist some chains $y_{i}$ in $X, 1 \leqslant i \leqslant p$, so that $\partial y_{1}=x_{0}$ and $\partial y_{i+1}=x_{i}+\left(1+c_{*}\right) y_{i}$ for $i \geqslant 1$. In this case $\operatorname{bv}_{p} x$ is represented by the class of $x_{p}+\left(1+c_{*}\right) y_{p}$ in $H_{p}(X)$

(3) ${ }^{1} E_{*}=H_{*}(X)$ and ${ }^{1} d_{*}=1+c_{*}$;

(4) ${ }^{r} d_{p}$ is defined on a cycle $x_{p}$ in $X$ if and only if there are chains $y_{p}=x_{p}, y_{p+1}$, $\ldots, y_{p+r-1}$ so that $\partial y_{i+1}=\left(1+c_{*}\right) y_{i}$. In this case ${ }^{r} d_{p} x_{p}=\left(1+c_{*}\right) y_{p+r-1}$.

A.2.1. Spectral sequence of involution. The homomorphisms $\mathrm{bv}_{*}$ and spectral sequence $\left({ }^{r} E_{*},{ }^{r} d_{*}\right)$ are natural with respect to equivariant maps. Furthermore, ${ }^{r} E_{*}$ and ${ }^{r} d_{*}$ do form a spectral sequence (i.e., ${ }^{r} d_{p}$ are well defined homomorphisms ${ }^{r} E_{p} \rightarrow{ }^{r} E_{p+r-1}$ and $\left.{ }^{r+1} E_{p}=\operatorname{Ker}^{r} d_{p} / \operatorname{Im}^{r} d_{p-r+1}\right)$, and this sequence converges to $H_{*}($ Fix $c)$ via $\mathrm{bv}_{*}$, i.e., $\mathrm{bv}_{p}$ induces an (honest) isomorphism $\mathcal{F}^{p} / \mathcal{F}^{p+1} \rightarrow{ }^{\infty} E_{p}$, where $\mathcal{F}^{p}=$ Domain $v_{p}=\operatorname{Ker~bv}_{p-1}$.

Remark. A detailed analysis of the construction above shows that $\mathrm{bv}_{p}$ can as well 
be regarded as a true homomorphism

$$
\mathrm{bv}_{p}: \operatorname{Ker}\left[\mathrm{bv}_{p-1}: H_{*}(\operatorname{Fix} c)-{ }^{r-1} E_{p-1}\right] \rightarrow{ }^{r} E_{p}, \quad r>1
$$

(where bv $v_{p}: H_{*}(\mathrm{Fix} c) \rightarrow{ }^{1} E_{p}=H_{p}(X)$ is defined as the inclusion homomorphism on $H_{p}($ Fix $c)$ and zero on $H_{>p}($ Fix $c)$ ). These homomorphisms are, in fact, related to the original, nonstabilized, Borel-Serre spectral sequence (see [Deg1] for details).

The cohomology version ${ }^{r} E^{*} \Rightarrow H^{*}($ Fix $c)$ is constructed similarly and is dual to the homology one. The cup- and cap-products in $X$ naturally descend to, respectively, a $\mathbb{Z}_{2}$-algebra structure in ${ }^{r} E^{*}$ and ${ }^{r} E^{*}$-module structure in ${ }^{r} E_{*}$. If $X$ is a connected closed $N$-manifold and Fix $c \neq \varnothing$, the fundamental class $[X]$ survives to ${ }^{\infty} E_{N}$ and the multiplication $\cap[X]:{ }^{r} E^{p} \rightarrow{ }^{r} E_{N-p}$ is an isomorphism (Poincaré duality). This, in the usual way, defines a pairing $\circ:{ }^{r} E_{p} \otimes{ }^{r} E_{q} \rightarrow{ }^{r} E_{p+q-N}$, called Kalinin's intersection pairing.

A.2.2. Kalinin's intersection pairing. Let $X$ be a smooth closed connected $N$-manifold with a smooth involution $c: X \rightarrow X$. Denote by $w(\nu)$ the total StiefelWhitney class of the normal bundle $\nu$ of Fix $c$ in $X$. Then for $a \in \mathcal{F}^{p}$ and $b \in \mathcal{F}^{q}$ one has $w(\nu) \cap(a \circ b) \in \mathcal{F}^{p+q-N}$ and

$$
\operatorname{bv}_{p} a \circ \operatorname{bv}_{q} b=\operatorname{bv}_{p+q-N}[w(\nu) \cap(a \circ b)] .
$$

Remark. The above identity contains, as a special case, the Borel-Haefliger [BH] version of the Bezout theorem over the reals (with the $\mathbb{Z}_{2}$-valued intersection): given two real algebraic cycles $A, B$ of complementary dimension in a real algebraic manifold $X$, their intersection number in $X$ is congruent mod 2 to the intersection number of $\mathbb{R} A$ and $\mathbb{R} B$ in $\mathbb{R} X$. To see this, it is sufficient to notice that $[\mathbb{R} A]$ is the highest degree term of $\mathrm{bv}^{-1}[A]$.

The homology Steenrod operations $\mathrm{Sq}_{t}: H_{*}(X) \rightarrow H_{*-t}(X)$ also descend to ${ }^{r} E_{*}$. In order to describe their relation to the ordinary Steenrod operations in $H_{*}($ Fix $c)$, introduce the weighed Steenrod operations

$$
\widehat{\mathrm{Sq}_{t}}: H_{p}(X) \rightarrow H_{\leqslant p}(X), \quad x \mapsto \sum_{0 \leqslant j \leqslant t}\left(\begin{array}{c}
P-p \\
t-j
\end{array}\right) \mathrm{Sq}_{j} x,
$$

where $P>p+t$ is a power of 2 . (The binomial coefficients do not depend on $P$, see, e.g., [SE, Lemma I.2.6].) Then one has:

A.2.3. Steenrod squares in Kalinin's spectral sequence. If $x \in \mathcal{F}^{p}$ and $t \geqslant 0$, then $\widehat{\operatorname{Sq}}_{t} x \in \mathcal{F}^{p-t}$ and

$$
\mathrm{Sq}_{t} \mathrm{bv}_{p} x=\mathrm{bv}_{p-t} \widehat{\operatorname{Sq}}_{t} x
$$

Kalinin's spectral sequence can alternatively be derived from the Smith exact sequence A.1.1. Below we give the corresponding description of the differentials ${ }^{r} d_{*}$ and Viro homomorphisms $\mathrm{bv}_{*}$; the construction of the spectral sequence and filtration $\mathcal{F}^{*}$ can be found in $\left.[\mathrm{DIK}].\right)$ 
A.2.4. Kalinin's spectral sequence and Smith exact sequence. The differentials ${ }^{r} d_{p}$ and Viro homomorphisms $\mathrm{bv}_{p}$ are given by

$$
\begin{aligned}
{ }^{r} d_{p}= & \operatorname{tr}^{p+r-1} \circ \Delta_{p+r-1}^{-1} \circ \ldots \circ \Delta_{p+1}^{-1} \circ \operatorname{pr}_{p}: H_{p}(X) \rightarrow H_{p+r-1}(X), \\
& \operatorname{bv}_{p}\left(\sum_{r \leqslant p} x_{i}\right)=\operatorname{in}_{p} x_{p}+\operatorname{tr}^{p} y_{p}^{\prime}: H_{*}(\text { Fix } c) \rightarrow H_{p}(X),
\end{aligned}
$$

where $\operatorname{in}_{*}: H_{*}($ Fix $c) \rightarrow H_{*}(X)$ is the inclusion homomorphism, $\operatorname{tr}^{*}, \mathrm{pr}_{*}$, and $\Delta_{*}$ are as in the Smith exact sequence A.1.1, and, in the latter equation, $x_{r} \in H_{r}(\mathrm{Fix} c)$ and $y_{p}^{\prime} \in H_{p}\left(X^{\prime}\right.$, Fix $\left.c\right)$ is defined recursively via $y_{0}^{\prime}=0, \quad y_{r+1}^{\prime}=\Delta_{r+1}^{-1}\left(x_{r} \oplus y_{r}^{\prime}\right) \in$ $H_{r+1}\left(X^{\prime}\right.$, Fix $\left.c\right)$.

A.2.5. Lifting involutions. Let $(X, c)$ be a topological space with involution, $Y \rightarrow X$ a double covering, and $\omega \in H^{1}(X)$ its characteristic class. Then $c$ lifts to an involution on $Y$ if and only if $\omega$ survives to ${ }^{\infty} E^{1}(X)$. If this is the case, there obviously are two lifts $c_{1}, c_{2}: Y \rightarrow Y$, which commute with each other and with the deck translation $\tau$ of the covering and satisfy the identity $c_{1} \circ c_{2}=\tau$.

An obvious necessary condition for $\omega$ to survive to ${ }^{\infty} E^{1}$ is that it must be $c^{*}$ invariant (i.e., survive to ${ }^{2} E^{1}$ ). Note that if $X$ is connected and Fix $c \neq \varnothing$, this condition is also sufficient as all the differentials landing in ${ }^{r} E^{0}$ are trivial.

Everything above applies to branched coverings as well, with $X$ replaced with $X \backslash A$, where $A$ is the branch set.

A.2.6. Complex surfaces. Kalinin's spectral sequence, as well as the related intersection pairing and Steenrod operations have a transparent geometrical meaning in the case when $X$ is a complex surface and $c=\operatorname{conj}$ a real structure. Let $C_{i}, i=1, \ldots, k$, be the components of $\mathbb{R} X,\left\langle C_{i}\right\rangle \in H_{0}(\mathbb{R} X)$ the corresponding classes, and $C_{I}=\sum_{i \in I}\left\langle C_{i}\right\rangle$ for $I \subset\{1, \ldots, k\}$. The values $b_{0}\left\langle C_{i}\right\rangle$, bv $1 \alpha$ (with $\alpha \in H_{1}(\mathbb{R} X)$ ), and $\mathrm{bv}_{2}\left[C_{i}\right]$ are always well defined and coincide with the images of the classes under the inclusion homomorphisms. The value $\mathrm{bv}_{1}\left\langle C_{I}\right\rangle$ is well defined whenever the number $|I|$ of summands is even; it is represented by the collection $\left(1+\operatorname{conj}_{*}\right) y_{1}$ of invariant circles, where $y_{1}$ is a collection of arcs in $X$ with one boundary point in each of the summands $C_{i}, i \in I$ (and no other boundary points). If, under an appropriate choice of $y_{1}$, the curve $\left(1+\operatorname{conj}_{*}\right) y_{1}$ is null homologous, $\left(1+\operatorname{conj}_{*}\right) y_{1}=\partial y_{2}$, then $\operatorname{bv}_{1}\left\langle C_{I}\right\rangle=0$ and $\left(1+\operatorname{conj}_{*}\right) y_{2}$ represents $\mathrm{bv}_{2}\left\langle C_{I}\right\rangle$. Similarly, if $\mathrm{bv}_{1} \alpha=0$, i.e., $\alpha$ is homologous to $\left(1+\operatorname{conj}_{*}\right) y_{1}$ for a chain $y_{1}$ in $X$, there is a chain $y_{2}$ in $X$ such that $\partial y_{2}=\alpha+\left(1+\operatorname{conj}_{*}\right) y_{1}$, and $\left(1+\operatorname{conj}_{*}\right) y_{2}$ represents $\mathrm{bv}_{2} \alpha$. Finally, if $\mathrm{bv}_{1} \alpha=\mathrm{bv}_{1}\left\langle C_{I}\right\rangle$ for some 0-class $C_{I}$ and 1-class $\alpha \in H_{1}(\mathbb{R} X)$, then $\operatorname{bv}_{2}\left(\alpha+\left\langle C_{I}\right\rangle\right)$ is defined and is represented by $\left(1+\operatorname{conj}_{*}\right) y_{2}$, where $\partial y_{2}=\alpha+\left(1+\operatorname{conj}_{*}\right) y_{1}$ and $y_{1}$ is an appropriately chosen collection of arcs with one boundary point in each of $C_{i}, i \in I$, and no other boundary points.

Elements of the form $\mathrm{bv}_{2}\left[C_{i}\right], \mathrm{bv}_{2}\left\langle C_{I}\right\rangle, \mathrm{bv}_{2} \alpha$, and $\mathrm{bv}_{2}\left(\alpha+\left\langle C_{I}\right\rangle\right)$ span ${ }^{\infty} E_{2}(X)$; with an abuse of the language we let $\sum \mathrm{bv}_{2} x_{j}=\mathrm{bv}_{2} \sum x_{j}$, provided that the latter is well defined, even if the summands are not. According to A.2.2, Kalinin's intersection form on ${ }^{\infty} E_{2}(X)$ is the one given by Table 1 , where the intersection $\alpha \circ \beta$ is regarded as an element of $H_{0}(\mathbb{R} X)$, and $\operatorname{aug}(\alpha \circ \beta)$ and $\operatorname{aug}_{i}(\alpha \circ \beta)$ are, respectively, the total intersection number and its portion falling into $C_{i}$. The notation $\delta_{i j}$ stands for the Kronecker symbol: $\delta_{i i}=1$ and $\delta_{i j}=0$ if $i \neq j$. The intersection form extends linearly to the classes of the form $\mathrm{bv}_{2}\left(\alpha+\left\langle C_{I}\right\rangle\right)$, as if $\mathrm{bv}_{2} \alpha$ and $\mathrm{bv}_{2}\left\langle C_{I}\right\rangle$ were well defined. 
TABLE 1. Kalinin's intersection form

\begin{tabular}{|c|c|c|c|}
\hline & $\mathrm{bv}_{2}\left\langle C_{I}\right\rangle$ & $\mathrm{bv}_{2} \alpha$ & $\mathrm{bv}_{2}\left[C_{i}\right]$ \\
\hline $\mathrm{bv}_{2}\left\langle C_{K}\right\rangle$ & 0 & 0 & $\sum_{k \in K} \delta_{i k}$ \\
\hline $\mathrm{bv}_{2} \beta$ & 0 & $\operatorname{aug}(\alpha \circ \beta)$ & $\operatorname{aug}_{i}(\beta \circ \beta)$ \\
\hline $\mathrm{bv}_{2}\left[C_{k}\right]$ & $\sum_{i \in I} \delta_{i k}$ & $\operatorname{aug}_{k}(\alpha \circ \alpha)$ & $\delta_{i k} \chi\left(C_{i}\right)$ \\
\hline
\end{tabular}

The Bockstein homomorphism $\mathrm{Sq}_{1}:{ }^{\infty} E_{2}(X) \rightarrow{ }^{\infty} E_{1}(X)$ is given by A.2.3: one has $\mathrm{Sq}_{1} \mathrm{bv}_{2}\left[C_{i}\right]=\mathrm{bv}_{1} w_{1}\left(C_{i}\right)$ and $\mathrm{Sq}_{1} \mathrm{bv}_{2}\left(\alpha+\left\langle C_{I}\right\rangle\right)=\mathrm{bv}_{1} \alpha$.

We conclude this section with a few examples of calculation of the filtration $\mathcal{F}^{*}$ and Viro homomorphisms $b_{*}$. The first example may serve as a model for regular complete intersections in $\mathbb{P}^{n}$ (see $[\mathrm{Ka}]$ ), the second one, as a local model for involutions on smooth manifolds.

A.2.7. Filtrations in $\mathbb{R P}^{n}$ (see $[\mathrm{Ka}]$ ). Let $X=\mathbb{P}^{n}$ and $c=$ conj. Then the filtrations $\mathcal{F}^{*}$ on $H_{*}\left(\mathbb{R} \mathbb{P}^{n}\right)$ and $\mathcal{F}_{*}$ on $H^{*}\left(\mathbb{R P}^{n}\right)$ are given by

$$
\mathcal{F}^{r}=\operatorname{Sq} H_{\geqslant r / 2}\left(\mathbb{R P}^{n}\right), \quad \mathcal{F}_{r}=\operatorname{Sq} H^{\leqslant r / 2}\left(\mathbb{R} \mathbb{P}^{n}\right),
$$

where Sq stands for both homology and cohomology total Steenrod squares.

A.2.8. Filtration in Thom spaces (see [DK1]). Let $\nu$ be an $m$-dimensional vector bundle over a finite cell complex $F$, and let $T$ and $\partial T$ be the associated disk and sphere bundles, respectively, supplied with the antipodal involution. Then the filtrations $\mathcal{F}^{*}$ on $H_{*}(F)$ and $\mathcal{F}_{*}$ on $H^{*}(F)$ associated with Kalinin's spectral sequence of pair $(T, \partial T)$ are given by

$$
\mathcal{F}^{p}=w(\nu)^{-1} \cap H_{\geqslant p-m}(F), \quad \mathcal{F}^{p}=w(\nu) \cup H^{\leqslant p-m}(F),
$$

where $w(\nu)=1+w_{1}(\nu)+w_{2}(\nu)+\ldots$ is the total Stiefel-Whitney class of $\nu$. The Viro homomorphisms are

$$
\begin{gathered}
\operatorname{bv}_{p}: \mathcal{F}^{p} \rightarrow H_{p}(T, \partial T), \quad x \mapsto \operatorname{th}^{-1}[w(\nu) \cap x]_{p-m}, \\
\mathrm{bv}^{p}: H^{p}(T, \partial T) \rightarrow H^{*}(F) / \mathcal{F}_{p-1}, \quad x \mapsto w(\nu) \cup \operatorname{th}^{-1} x,
\end{gathered}
$$

where th is the Thom isomorphism and $[x]_{r}$ denotes the $r$-dimensional component of a nonhomogeneous homology class $x$.

Next statement is a generalization of A.2.7; it can be deduced from A.2.7 using the splitting principle.

A.2.9. Filtrations in Grassmann manifolds. Let $X=G_{k}\left(\mathbb{C}^{n}\right)$ be the Grassmann manifold of $k$-planes in $\mathbb{C}^{n}$ and $c=$ conj the standard conjugation. Then the filtrations $\mathcal{F}^{*}$ on $H_{*}\left(G_{k}\left(\mathbb{R}^{n}\right)\right)$ and $\mathcal{F}_{*}$ on $H^{*}\left(G_{k}\left(\mathbb{R}^{n}\right)\right)$ are given by

$$
\mathcal{F}^{r}=\operatorname{Sq} H_{\geqslant r / 2}\left(G_{k}\left(\mathbb{R}^{n}\right)\right), \quad \mathcal{F}_{r}=\operatorname{Sq} H^{\leqslant r / 2}\left(G_{k}\left(\mathbb{R}^{n}\right)\right),
$$

where Sq stands for both homology and cohomology total Steenrod squares.

The following consequence of A.2.9 is, in fact, equivalent to it. A related identity is found in $[\mathrm{Kr} 2]$. 
A.2.10. Corollary. Let $X$ be a topological space with involution $c: X \rightarrow X$ and $E$ a real vector bundle over $X$ in the sense of Atiyah [At] (i.e., $E$ is a $\mathbb{C}$-vector bundle supplied with an anti-linear involutive lift $c_{E}$ of $c$ ). Then for all $i$ one has $\mathrm{bv}^{2 i} c_{i}(E)=\mathrm{Sq} w^{i}(\mathbb{R} E)$, where $\mathbb{R} E=$ Fix $c_{E}$ is an $\mathbb{R}$-vector bundle over Fix $c$.

A.3. Involutions on manifolds. Let $X$ be a smooth closed oriented $2 k$-manifold and $c$ a smooth involution, preserving the orientation if $k$ is even and reversing it otherwise. Define the twisted intersection form

$$
B^{c}:\left(H_{k}(X ; \mathbb{Z}) / \text { Tors }\right) \otimes\left(H_{k}(X ; \mathbb{Z}) / \text { Tors }\right) \rightarrow \mathbb{Z}, \quad B^{c}(x, y)=x \circ c_{*} y .
$$

This form is symmetric; its signature $\sigma(c)$ is called the signature of the involution. If $k$ is odd, the signature is 0 , otherwise $\sigma(c)=\sigma_{+}-\sigma_{-}$, where $\sigma_{ \pm}$are the signatures of the restrictions of the intersection form to the eigenspaces $H_{k}^{ \pm 1}$ of $c_{*}$.

A.3.1. Theorem. If all the components of $F=$ Fix $c$ are of dimension $k$, one has $\sigma(c)=F \circ F$, where $F \circ F$ is the normal Euler number of $F$ in $X$.

Since the real part $\mathbb{R} X$ of a complex manifold $X$ with real structure is totally real, all the components of $\mathbb{R} X$ are of dimension $n=\operatorname{dim}_{\mathbb{C}} X$ and $\mathbb{R} X \circ \mathbb{R} X=$ $(-1)^{n / 2} \chi(\mathbb{R} X)$. If $X$ is supplied with an invariant Kähler metric, the real part is Lagrangian.

A.3.2. Corollary. If $X$ is a $2 k$-dimensional compact complex manifold with real structure conj, one has $\sigma($ conj $)=(-1)^{k} \chi(\mathbb{R} X)$.

If $X$ is a closed compact (not necessarily orientable) manifold and $\operatorname{dim} X=2 k$ is even, one can define a $\mathbb{Z}_{2}$-version $B^{c}: H_{k}(X) \otimes H_{k}(X) \rightarrow \mathbb{Z}_{2}$ of the twisted intersection form. It is also symmetric.

A.3.3. Arnol'd lemma. Let $X$ be a closed smooth manifold with involution $c$ and $\operatorname{dim} X=2 k$ even. If $\operatorname{dim}$ Fix $c \leqslant k$, then the fundamental class [Fix $c]_{k}$ of the union of the $k$-dimensional components of Fix $c$ realizes in $H_{k}(X)$ the characteristic class of $B^{c}: H_{k}(X) \otimes H_{k}(X) \rightarrow \mathbb{Z}_{2}$.

Remark. In general the characteristic class of $B^{c}$ is the $k$-dimensional component of $\operatorname{in}_{!}\left(v(\tau) v^{-1}(\nu)\right)$, where in: Fix $c \rightarrow X$ is the inclusion, $\tau$ and $\nu$ are, respectively, the tangent and normal bundles of Fix $c$, and $v$ stands for the total Wu class. See [CM] for details.

\section{Appendix B. Integral Lattices And QUADRATIC FORMS}

In this appendix we give a brief introduction into arithmetic of integral lattices and finite quadratic forms, which is a commonly used tool in topology of real algebraic varieties. Proofs, mostly straightforward, can be found in any advanced textbook, e.g., $[\mathrm{MH}]$ and $[\mathrm{S}]$.

B.1. Finite quadratic forms. A finite symmetric bilinear form is a pair $(\mathcal{L}, b)$, where $\mathcal{L}$ is a finite abelian group and $b: \mathcal{L} \otimes \mathcal{L} \rightarrow \mathbb{Q} / \mathbb{Z}$ is a symmetric bilinear form. A finite quadratic form on $\mathcal{L}$ is a map $q: \mathcal{L} \rightarrow \mathbb{Q} / 2 \mathbb{Z}$ satisfying the relations $q(n x)=n^{2} q(x), x \in \mathcal{L}, n \in \mathbb{Z}$, and $q(x+y)-q(x)-q(y)=2 b(x, y), x, y \in \mathcal{L}$, where $b$ is a symmetric bilinear form; $b$ is determined by $q$ and is called the bilinear form associated with $q$, whereas $q$ is called a quadratic extension of $b$. 
If a quadratic form $q$ (bilinear form $b$ ) is understood, it is often abbreviated as $q(x)=x^{2}$ (respectively, $\left.b(x, y)=x y\right)$.

If $q_{1}, q_{2}$ are two quadratic extensions of the same bilinear form $b$, the difference $q_{1}-q_{2}$ is a linear form. Furthermore, for any $x \in \mathcal{L}$ one has $q_{i}(x)=b(x, x) \bmod \mathbb{Z}$, $i=1,2$; hence, $q_{1}-q_{2}$ takes values in $\mathbb{Z}_{2}=\mathbb{Z} / 2 \mathbb{Z} \subset \mathbb{Q} / 2 \mathbb{Z}$. Thus, the set $\mathrm{Qu}(\mathcal{L}, b)$ of quadratic extensions of a given bilinear form $b$ on $\mathcal{L}$ is an affine space over $\operatorname{Hom}\left(\mathcal{L}, \mathbb{Z}_{2}\right)$. In particular, unless $\mathcal{L}$ has 2 -torsion, any bilinear form $b$ on $\mathcal{L}$ has a unique quadratic extension.

A symmetric bilinear form $b$ is called nondegenerate if the correlation homomorphism $\mathcal{L} \rightarrow \operatorname{Hom}(\mathcal{L}, \mathbb{Q} / \mathbb{Z}), x \mapsto b(x, \cdot)$, is bijective. A quadratic form is called nondegenerate if so is its associated bilinear form. In general, the kernel of the correlation homomorphism is denoted by $\operatorname{Ker} \mathcal{L}$ or $\mathcal{L}^{\perp}$.

The nondegenerate finite quadratic and bilinear forms constitute monoids Bil and $\mathrm{Qu}$, respectively, the addition operation being the orthogonal sum. A set of generators of Bil is given, e.g., in [Wl]; for each generator $\mathcal{L}$ is either cyclic or (not orthogonal) sum of two cyclic groups. Generators of $\mathrm{Qu}$ can be obtained by lifting those of Bil. In this survey we deal explicitly only with forms on groups of period 2 (however, proofs of the results on real Enriques surfaces make an extensive use of forms on groups of period 4, whose theory can be found in [DIK]); they constitute direct summands $\mathrm{Bil}_{2}$ and $\mathrm{Qu}_{2}$ of $\mathrm{Bil}$ and $\mathrm{Qu}$, respectively. The Wall generators of $\mathrm{Bil}_{2}$ are the following:

$$
\mathcal{L}=\mathbb{Z}_{2}: \quad a_{2}=\left[\frac{1}{2}\right] ; \quad \mathcal{L}=\mathbb{Z}_{2} \times \mathbb{Z}_{2}: \quad u_{2}=\left[\begin{array}{cc}
0 & \frac{1}{2} \\
\frac{1}{2} & 0
\end{array}\right] .
$$

Up to isomorphism $a_{2}$ and $u_{2}$ have two quadratic extensions each:

$$
\text { for } a_{2}: \quad\left[ \pm \frac{1}{2}\right], \quad \text { for } u_{2}: \quad \mathcal{U}_{2}=\left[\begin{array}{cc}
0 & \frac{1}{2} \\
\frac{1}{2} & 0
\end{array}\right] \text { and } \mathcal{V}_{2}=\left[\begin{array}{cc}
1 & \frac{1}{2} \\
\frac{1}{2} & 1
\end{array}\right]
$$

The Brown invariant $\operatorname{Br} q$ of a nondegenerate finite quadratic form $q$ on $\mathcal{L}$ is the $(\bmod 8)$-residue defined via

$$
\exp \left(\frac{1}{4} i \pi \operatorname{Br} q\right)=|\mathcal{L}|^{-\frac{1}{2}} \sum_{x \in \mathcal{L}} \exp (i \pi q(x)) .
$$

B.1.1. One has $\operatorname{Br}\left[ \pm \frac{1}{2}\right]= \pm 1, \operatorname{Br} \mathcal{U}_{2}=0$, and $\operatorname{Br} \mathcal{V}_{2}=4$.

A finite group $\mathcal{L}$ of period 2 is a $\mathbb{Z}_{2}$-vector space. If $\mathcal{L}$ is equipped with a finite quadratic form, it is called a quadratic space. A quadratic (bilinear) form on a $\mathbb{Z}_{2}$-space takes values in $\left(\frac{1}{2} \mathbb{Z}\right) / 2 \mathbb{Z} \subset \mathbb{Q} / 2 \mathbb{Z}$ (respectively, in $\left(\frac{1}{2} \mathbb{Z}\right) / \mathbb{Z} \subset \mathbb{Q} / \mathbb{Z}$ ). By means of the canonical identifications $\left(\frac{1}{2} \mathbb{Z}\right) / 2 \mathbb{Z}=\mathbb{Z}_{4}$ and $\left(\frac{1}{2} \mathbb{Z}\right) / \mathbb{Z}=\mathbb{Z}_{2}, \frac{1}{2} \mapsto 1$, one can consider quadratic (bilinear) forms on $\mathbb{Z}_{2}$-spaces as maps $\mathcal{L} \rightarrow \mathbb{Z}_{4}$ (respectively, $\left.\mathcal{L} \otimes \mathcal{L} \rightarrow \mathbb{Z}_{2}\right)$.

A vector $w \in \mathcal{L}$ is called a characteristic element of $\mathcal{L}$ if $x w=x^{2} \bmod 2$ for any $x \in \mathcal{L}$. Since on groups of period 2 the map $x \mapsto x^{2} \bmod 2$ is linear, characteristic elements exist and form a coset $\bmod (\operatorname{Ker} \mathcal{L})$; it is called the characteristic coset of $\mathcal{L}$. If $\mathcal{L}$ is nondegenerate, a characteristic element is unique. The subgroup of $\mathcal{L}$ spanned by $\operatorname{Ker} \mathcal{L}$ and a characteristic element will be denoted by $\Omega=\Omega(\mathcal{L})$.

A quadratic space is called even if 0 is its characteristic element, i.e., if for all $x \in \mathcal{L}$ one has $x^{2}=0 \bmod 2$. Otherwise, it is called odd. 
B.1.2. The monoid of nondegenerate finite quadratic forms on groups of period 2 is generated by $\mathcal{A}^{ \pm}=\left[ \pm \frac{1}{2}\right], \mathcal{U}_{2}$, and $\mathcal{V}_{2}$, which are subject to the relations $2 \mathcal{U}_{2}=2 \mathcal{V}_{2}$, $3 \mathcal{A}^{ \pm}=\mathcal{A}^{\mp} \oplus \mathcal{V}_{2}$, and $\mathcal{A}^{ \pm} \oplus \mathcal{A}^{+} \oplus \mathcal{A}^{-}=\mathcal{A}^{ \pm} \oplus \mathcal{U}_{2}$.

Two nondegenerate quadratic spaces are isomorphic if and only if they have the same rank, parity, and Brown invariant.

A quadratic form $q$ on $\mathcal{L}$ is called informative if $\left.q\right|_{\mathcal{L}^{\perp}}=0$. The notion of Brown invariant extends to informative forms: since $q$ vanishes on $\mathcal{L}^{\perp}$, it descends to a quadratic form $q^{\prime}: \mathcal{L} / \mathcal{L}^{\perp} \rightarrow \mathbb{Q} / 2 \mathbb{Z}$, and one lets $\operatorname{Br} q=\operatorname{Br} q^{\prime}$. The Brown invariant is additive: given a pair $\left(\mathcal{L}_{i}, q_{i}\right), i=1,2$, of informative quadratic forms, one has $\operatorname{Br}\left(\mathcal{L}_{1} \oplus \mathcal{L}_{2}, q_{1} \oplus q_{2}\right)=\operatorname{Br}\left(\mathcal{L}_{1}, q_{1}\right)+\operatorname{Br}\left(\mathcal{L}_{2}, q_{2}\right)$.

A subform $\mathcal{N}$ of an informative quadratic form $(\mathcal{L}, q)$ is called informative if $\mathcal{N}^{\perp} \subset \mathcal{N}$ and $\left.q\right|_{\mathcal{N}^{\perp}}=0$. (Clearly, an informative subform is an informative form; hence, its Brown invariant is well defined.) A subform $\mathcal{S} \subset \mathcal{L}$ is called isotropic (more precisely, $q$-isotropic) if $\left.q\right|_{\mathcal{S}}=0$. Clearly, a subform $\mathcal{N}$ of a nondegenerate form $(\mathcal{L}, q)$ is informative if and only if $\mathcal{N}^{\perp}$ is isotropic.

The following is a direct consequence of a Gauss summation formula.

B.1.3. If $\mathcal{S}$ is a $q$-isotropic subform of a nondegenerate quadratic form $(\mathcal{L}, q)$, then $q$ descends to a nondegenerate quadratic form on $\mathcal{S}^{\perp} / \mathcal{S}$ and $\operatorname{Br}\left(S^{\perp} / S\right)=\operatorname{Br} \mathcal{L}$.

B.1.4. Corollary. If $\mathcal{N}$ is an informative subform of an informative quadratic form $(\mathcal{L}, q)$, then $\operatorname{Br}\left(\mathcal{N},\left.q\right|_{\mathcal{N}}\right)=\operatorname{Br}(\mathcal{L}, q)$.

Remark. The notion of informative subspace still makes sense if the quadratic form $q$ is only defined on $\mathcal{N}$ (while the bilinear form is defined on the whole space $\mathcal{L}$ ). In this case Corollary B.1.4 states that $\operatorname{Br} q^{\prime}=\operatorname{Br} q$ for any extension $q^{\prime}$ of $q$ to $L$.

B.1.5. For any informative quadratic space $(\mathcal{L}, q)$ one has:

(1) $\operatorname{Br} q=\operatorname{dim}\left(\mathcal{L} / \mathcal{L}^{\perp}\right) \bmod 2$;

(2) $\operatorname{Br} q=q(w) \bmod 4$ for any characteristic element $w \in \mathcal{L}$;

(3) $\operatorname{Br}(q+v)=\operatorname{Br} q-2 q(v)$ for any $v \in \mathcal{L}$ (where $q+v$ stands for the quadratic form $x \mapsto q(x)+2(v x))$;

(4) $\operatorname{Br} q=0$ if and only if $(\mathcal{L}, q)$ is null cobordant, i.e., there is a subspace $\mathcal{H} \subset \mathcal{L}$ such that $\mathcal{H}^{\perp}=\mathcal{H}$ and $\left.q\right|_{\mathcal{H}}=0$.

B.2. Integral lattices. An (integral) lattice is a free abelian group $L$ of finite rank supplied with a symmetric bilinear form $b: L \otimes L \rightarrow \mathbb{Z}$. As in the case of finite forms, we often abbreviate $b(x, y)=x y$ and $b(x, x)=x^{2}$. A lattice $L$ is called even if $x^{2}=0 \bmod 2$ for all $x \in L$; otherwise, $L$ is called odd. Let $L^{\vee}=\operatorname{Hom}(L, \mathbb{Z})$ be the dual abelian group. $L$ is called nondegenerate (unimodular) if the correlation homomorphism $L \rightarrow L^{\vee}, x \mapsto b(x, \cdot)$, is monic (respectively, one-to-one). The cokernel of the correlation homomorphism is called the discriminant group of $L$ and denoted by $\operatorname{discr} L$ or $\mathcal{L}$.

The bilinear form on $L$ extends to a $\mathbb{Q}$-valued bilinear form on $L \otimes \mathbb{Q}$. If $L$ is nondegenerate, there is a natural identification $L^{\vee}=\{x \in L \otimes \mathbb{Q} \mid x y \in \mathbb{Z}$ for $y \in L\}$ and the correlation homomorphism is the embedding $L \hookrightarrow L^{\vee}$. Hence, $\mathcal{L}$ is finite and the form on $L$ extends to a $\mathbb{Q}$-valued form on $L^{\vee}$, which, in turn, induces a finite symmetric bilinear form $b: \mathcal{L} \otimes \mathcal{L} \rightarrow \mathbb{Q} / \mathbb{Z}$. The order of $\mathcal{L}$ is equal to the absolute value of the determinant $\operatorname{det} L$ of the Gram matrix of $L$. If, in addition, $L$ is even, $b$ is associated with the finite quadratic form $q: \mathcal{L} \rightarrow \mathbb{Q} / 2 \mathbb{Z}$ defined via $q(x+L)=x^{2} \bmod 2 \mathbb{Z}, x \in L^{\vee}$. 
B.2.1. Van der Blij formula. For a nondegenerate even integral lattice $L$ one has $\operatorname{Br} \mathcal{L}=\sigma(L) \bmod 8$.

The following proposition is an immediate consequence of B.2.1:

B.2.2. Corollary. Let $L$ be an even nondegenerate integral lattice whose discriminant group $\mathcal{L}=\operatorname{discr} L$ is of period 2. Then:

(1) if $L$ is unimodular (i.e., $\mathcal{L}=0$ ), then $\sigma(L)=0 \bmod 8$;

(2) if $\operatorname{det} L= \pm 2$ (i.e., $\mathcal{L}=\mathbb{Z}_{2}$ ), then $\sigma(L)= \pm 2 \bmod 8$;

(3) if $\operatorname{det} L= \pm 4$ (i.e., $\mathcal{L}=\mathbb{Z}_{2} \oplus \mathbb{Z}_{2}$ ) and the discriminant form on $\mathcal{L}$ is odd, then $\sigma(L)=0, \pm 2 \bmod 8$

(4) if the discriminant form on $\mathcal{L}$ is even, then $\sigma(L)=0 \bmod 4$.

B.3. Involutions on unimodular lattices. Assume that $L$ is a unimodular lattice and $c: L \rightarrow L$ an involutive auto-isometry of $L$. Let $L^{ \pm} \subset L$ be the eigenlattices of $c, q^{ \pm}=\operatorname{discr} L^{ \pm}$, and $J=L /\left(L^{+} \oplus L^{-}\right)$. As groups, $J$ and $q^{ \pm}$are isomorphic $\mathbb{Z}_{2}$-vector spaces. The eigenlattices are the orthogonal complements of each other and one has $\mathrm{rk} L^{+}+\operatorname{rk} L^{-}=\operatorname{rk} L$ and

$$
\log _{2}\left|\operatorname{discr} L^{ \pm}\right|=\operatorname{dim}_{\mathbb{Z}_{2}} J=\operatorname{dim}_{\mathbb{Z}_{2}} L \otimes \mathbb{Z}_{2}-\operatorname{dim}_{\mathbb{Z}_{2}}\left\{L \otimes \mathbb{Z}_{2}\right\}^{c},
$$

where $\left\{L \otimes \mathbb{Z}_{2}\right\}^{c}=\left\{x \in L \otimes \mathbb{Z}_{2} \mid c x=l\right\}$.

Next statement is straightforward:

B.3.1. The form $q^{+}$is even if and only if the characteristic elements of $L$ coincide with those of the twisted form $b^{c}: L \otimes L \rightarrow \mathbb{Z}_{2}, x \otimes y \mapsto b(x, c y)$.

\section{Appendix C. The Rokhlin-Guillou-Marin form}

In this appendix we briefly remind the construction of the Rokhlin-Guillou-Marin form (see $[\mathrm{GM}]$ ) of a characteristic surface in a 4-manifold.

Let $Y$ be an oriented closed smooth 4-manifold and $U$ a characteristic surface in $Y$, i.e., a smooth closed 2-submanifold with $[U]=\mathrm{D} u_{2}(Y)$ in $H_{2}(Y)$. Denote by $i: U \hookrightarrow Y$ the inclusion and let $K=\operatorname{Ker}\left[i_{*}: H_{1}(U) \rightarrow H_{1}(Y)\right]$. Then there is a natural quadratic extension $\mathfrak{g m}: K \rightarrow \mathbb{Z}_{4}$ of the intersection index form, which is called the Rokhlin-Guillou-Marin form of $(Y, U)$. It can be defined as follows. Pick a class $x \in K$ and realize it by a union $\mathfrak{l}$ of disjoint simple closed smooth loops in $U$. It spans an immersed surface $\mathfrak{M}$ in $Y$, which can be chosen normal to $U$ along $\mathfrak{l}=\partial \mathfrak{M}$ and transversal to $U$ at its inner points. (Such a surface is called a membrane.) Consider a normal line field $\xi$ on $\mathfrak{l}$ tangent to $U$ and define the index ind $\mathfrak{M} \in \mathbb{Z}$ as the obstruction to extending $\xi$ to a normal line field on $\mathfrak{M}$. (Since $\tau \mathfrak{M} \oplus \nu \mathfrak{M}$ is an oriented vector bundle, the obstruction is a well defined integer. If $\mathfrak{l}$ is two-sided in $U$, the index equals twice the index defined via vector fields instead of line fields.) Then $\mathfrak{g m}(x)=$ ind $\mathfrak{M}+2$ Card(int $\mathfrak{M} \cap F) \bmod 4$.

C.0.1. Theorem. Let $Y, U$, and $(K, \mathfrak{g m})$ be as above. Then $(K, \mathfrak{g m})$ is an informative subspace of $H_{1}(U)$ and

$$
2 \operatorname{Br} \mathfrak{g m}=\sigma(Y)-U \circ U \bmod 16,
$$

where $U \circ U$ stands for the normal Euler number of $U$ in $Y$.

For the proof in the case when $Y$ is simply connected (or, more generally, when $K=H_{1}(U)$ ) see [GM], for the general case see, e.g., [DIK]. Rokhlin considered the 
case when the characteristic surface is orientable and used the Arf-invariant: in this case $4 \mathrm{Arf}=\mathrm{Br}$.

Remark. There is an alternative construction of the Rokhlin-Guillou-Marin form. Since $U$ is characteristic, $Y \backslash U$ admits a Spin-structure which does not extend through any component of $U$. Its restriction to the boundary of a tubular neighborhood of $U$ induces in a natural way a $\mathrm{Pin}^{-}$-structure on $U$ (cf. [F3]), which defines a quadratic form $q$ on $H_{1}(U)$. It is not difficult to see that $q$ is well defined up to adding elements of $\operatorname{Im}\left[i^{*}: H^{1}(Y) \rightarrow H^{1}(U)\right]$ and, hence, its restriction to $K$

does not depend on the choice of a Spin-structure; it coincides with $\mathfrak{g m}$. (This gives an alternative proof of C.0.1, communicated to us by C. Bailly: the quadratic forms arising from $\mathrm{Pin}^{-}$-structures are isomorphic to each other since they have the same Brown-invariant (see B.1.2); these forms provide all the quadratic extensions of $\mathfrak{g m}$ from $K$ to $H_{1}(U)$ and, as it is shown in [Ba], this implies that $K$ is informative.)

\section{REFERENCES}

[Ak] S. Akbulut, On quotients of complex surfaces under complex conjugation, J. Reine Angew. Math. 447 (1994), 83-90.

[A1] V. I. Arnold, On arrangement of ovals of real plane algebraic curves, the involutions of four-dimensional smooth manifolds, and the arithmetic of integral quadratic forms, Funkc. Anal. i Prilozhen. 5 (1971), no. 3, 1-9.

[A2] V. I. Arnold, The index of a singular point of a vector field, the Petrovkii-Oleinik inequalities and mixed Hodge structures, Funkc. Anal. i Prilozhen. 12 (1978), no. 1, 1-14.

[A3] V. I. Arnold, The branched covering $\mathbb{C} P^{2} \rightarrow S^{4}$, hyperbolocity and projective topology, Sibirsk. Mat. Zh. 29 (1988), no. 5, 36-47.

[A4] V. I. Arnold, Topological content of the Maxwell theorem on multipole representation of spherical functions, Topol. Methods Nonlinear Anal. 7 (1996), no. 2, 205-217.

[AO] V. I. Arnold, O. A. Oleinik, Topology of real algebraic manifolds, Vestnik Mosk. Univ., Ser. I, Mat. i Mekh. 6 (1979), 7-17.

[At] M. F. Atiyah, K-theory and reality, Quart. J. Math. Oxford Ser. (2) 17 (1966), 367-386.

[AD] M. F. Atiyah, J. L. Dupont, Vector fields with finite singularities, Acta Math. Stockh. 128 (1972), 1-40.

[Ba] C. Bailly, A. Vdovina, Sous-espaces déterminant l'invariant de Arf et un Théorème de Rokhlin sur la signature (to appear).

[Bi] F. Bihan, Constructions Combinatoires de Surfaces Algébriques Réelles, Thèse, 1998.

[BPV] W. Barth, C. Peters, A. van de Ven, Compact complex surfaces, Ergebnisse der Mathemtik und ihrer Grenzgebiete (3), Springer-Verlag, Berlin-New York, 1984.

[BH] A. Borel, A. Haefliger, La classe d'homologie fondamentale d'un espace analytique, Bull. Soc. math. France 89 (1961), 461-513.

[Br] G. E. Bredon, Introduction to compact transformation groups, Academic Press, New YorkLondon, 1972.

[C] J. Cerf, Sur les difféomorphismes de la sphére de dimension trois, $\Gamma_{4}=0$, Lecture Notes Math. 53.

[CP] C. Ciliberto, C. Pedrini, Annibale Comessatti and real algebraic geometry, R.C. Circolo Mat. Palermo. Ser. II. Suppl. no. 36 (1994), 71-102.

[Ch] S. S. Chern, Complex manifolds, Russian translation, Izd. Inostr. Lit., Moscow, 1961.

[Co1] A. Comessatti, Sulla connessione delle superficie algebriche reali, Verhandl. Intern. Math. Kongr. Zürich, vol. 2, pp. 169.

[Co2] A. Comessatti, Fondamenti per la geometria sopra le superficie razionali dal punto di vista reale, Math. Annalen 43 (1912), 1-72.

[Co3] A. Comessatti, Sur la connessione delle superficie razionali reale, Annali di Matematica 23 (1914), 215-285.

[Co4] A. Comessatti, Reele Fragen in der algebraischen Geometrie, Jahresbericht d. Deut. Math. Vereinigung 41 (1932), 107-134.

[CM] P. E. Conner, E. Y. Miller, Equivariant self-intersection, Preprint, 1979. 
[DKh] V. I. Danilov, A. G. Khovanskii, Newton polygons and an algorithm for computing HodgeDeligne numbers, Izv. Akad. Nauk SSSR, Ser. Matem. 50 (1986), no. 5, 925-945; English transl., Math. USSR Izv. 29 (1987), 279-298.

[Deg1] A. Degtyarev, Stiefel orientations on a real algebraic variety, Proc. Rennes Symp. on Real Alg. Geom., Lect. Notes in Math., vol. 1524, 1992, pp. 205-220.

[Deg2] A. Degtyarev, Cohomology approach to killing structures on Steenrod bundles, Geometry and Topology, I, Adv. Soviet Math., vol. 18, 1994, pp. 1-22.

[Deg3] A. Degtyarev, On the Pontrjagin-Viro form, Advances Math. Sciences, Rokhlin's Memorial volume (to appear).

[DIK] A. Degtyarev, I. Itenberg, V. Kharlamov, Real Enriques Surfaces (to appear).

[DK1] A. Degtyarev, V. Kharlamov, Halves of a real Enriques Surface, Comment. Math. Helvetici 71 (1996), 628-663.

[DK2] A. Degtyarev, V. Kharlamov, Topological classification of real Enriques surfaces, Topology 35 (1996), no. 3, 711-729.

[DZ] A. Degtyarev, V. I. Zvonilov, Rigid isotopy classification of real algebraic curves of bidegree $(3,3)$ on quadrics, Mat. Zametki 66 (1999), no. 6, 810-815.

[Don] S. Donaldson, Yang-Mills invariants of smooth four-manifolds, Geometry of low-dimensional manifolds, vol. 1, Cambridge Univ. Press, 1990, pp. 5-40.

[Ed] A. L. Edmonds, Orientability of fixed point sets, Proc. Amer. Math. Soc. 82 (1981), no. 1, 120-124.

[EKh] Ya. M. Eliashberg, V. Kharlamov, On the number of complex points of a real surface in a complex one, Proceedings of Leningrad International Topology conference, 1982, Leningrad, pp. 143-148.

[Fi1] T. Fiedler, Pencils of lines and the topology of real algebraic curves, Izv. Akad. Nauk SSSR Ser. Mat. 46 (1982), no. 4, 853-863.

[Fi2] T. Fiedler, New congruences in the topology of real plane algebraic curves, Dokl. Akad. Nauk SSSR 270 (1983), no. 1, 56-58.

[Fi3] T. Fiedler, Additional inequalities in the topology of real plane algebraic curves, Izv. Akad. Nauk SSSR Ser. Mat. 49 (1985), no. 4, 874-883.

[F1] S. Finashin, Rokhlin conjecture and quotients of complex surfaces by complex conjugation, J. Reine Angew. Math. 481 (1996), 55-71.

[F2] S. Finashin, Decomposability of quotients by complex conjugation for rational and Enriques surfaces, Topology Appl. 79 (1997), no. 2, 121-128.

[F3] S. Finashin, A $\mathrm{Pin}^{-}$cobordism invariant and a generalization of the Rokhlin signature congruence, Algebra i Analiz 2 (1990), no. 4, 242-250.

[FS] S. Finashin, E. Shustin, On imaginary plane curves and Spin-quotients of complex surfaces by complex conjugation, AMS Translations, Ser. 2180 (1997), 93-101.

[G1] D. A. Gudkov, Construction of a new series of $M$-curves, Dokl. Akad. Nauk SSSR 200 (1971), no. 6, 1269-1272.

[G2] D. A. Gudkov, The topology of real projective algebraic varieties, Uspekhi. Matem. Nauk 29 (1974), no. 4, 3-79.

[G3] D. A. Gudkov, Topology of algebraic curves on a hyperboloid, Uspekhi Mat. Nauk 34 (1979), no. 6 (210), 26-32 (Russian); English transl. in Russian Math. Surveys 34 (1979).

[GS] D. A. Gudkov, E. I. Shustin, Classification of nonsingular eight-order curves on an ellipsoid, Methods of the Qualitative Theory of Differential Equations (E. A. LeontovichAndronova, ed.), Gor'kov. Gos. Univ., Gor'ki, 1980, pp. 104-107. (Russian)

[GU] D. A. Gudkov, A. K. Usachev, Nonsingular curves of small orders on a hyperboloid, Methods of the Qualitative Theory of Differential Equations (E. A. Leontovich-Andronova, ed.), Gor'kov. Gos. Univ., Gor'ki, 1980, pp. 96-103. (Russian)

[GUt] D. A. Gudkov, G. A. Utkin, Topology of curves of order 6 and surfaces of order 4, Uch. Zapiski Gor'kov. Univ., vol. 87, 1969.

[GM] L. Guillou, A. Marin, Une extension d'in théorème de Rohlin sur la signature, C. R. Acad. Sci. Paris 285 (1977), A95-A98.

[Ha] R. Hartshorne, Algebraic Geometry, Graduate Text in Math., vol. 49, Springer-Verlag, 1977.

[H1] F. Hirzebruch, Über vierdimensionale Riemannsche Flächen mehrdeufiger analitischer Functionen von zwei komplexen Veränderlichen, Math. Ann. 126 (1953), no. 1, 1-22.

[H2] F. Hirzebruch, Topological Methods in Algebraic Geometry, Springer-Verlag, 1978. 
[Ho] E. Horikawa, On deformations of quintic surfaces, Invent. Math. 31 (1975), 43-85.

[IKh] I. Itenberg, V. Kharlamov, Towards the maximal number of components of a nonsingular surface of degree 5 in $\mathbb{R P}^{3}$, Amer. Math. Soc. Trans. (2) 173 (1996), 111-118.

[IV1] I. Itenberg, O. Ya. Viro, Patchworking algebraic curves disproves the Ragsdale conjecture, Math. Intelligencer 18 (1996), no. 4, 19-28.

[IV2] I. Itenberg, O. Ya. Viro, Maximal real algebraic hypersurfaces of projective space, preprint.

[Ka] I. Kalinin, Cohomology characteristics of real algebraic hypersurfaces, Algebra i Analis 3 (1991), 313-332. (Russian)

[Kh1] V. Kharlamov, The maximal number of components of a 4 th degree surface in $\mathbb{R P}^{3}$, Funkc. Anal. i Prilozhen. 6 (1972), no. 4, 101.

[Kh2] V. Kharlamov, A generalized Petrovskii inequality, Funkc. Anal. i Prilozhen. 8 (1974), no. 2, 50-56 (Russian); English transl. in Functional Anal. Appl. 8 (1974), no. 2, 132-137.

[Kh3] V. Kharlamov, The topological type of nonsingular surfaces in $\mathbb{R P}^{3}$ of degree 4, Funkc. Anal. i Prilozhen. 10 (1976), no. 4, 55-68 (Russian); English transl. in Functional Anal. Appl. 10 (1976), no. 4, 295-305.

[Kh4] V. Kharlamov, A generalized Petrovskii inequality. II, Funkc. Anal. i Prilozhen. 9 (1975), no. 3, 93-94 (Russian); English transl. in Functional Anal. Appl. 9 (1975), no. 3, 266-268.

[Kh5] V. Kharlamov, Additional congruences for the Euler characteristic of even-dimensional real algebraic varieties, Funkc. Anal. i Prilozhen. 9 (1975), no. 2, 51-60.

[Kh6] V. Kharlamov, Isotopy types of non-singular surfaces of degree 4 in $\mathbb{R P}^{3}$, Funct. Anal. Appl. 12 (1978), 86-87.

[Kh7] V. Kharlamov, On the classification of non-singular surfaces of degree 4 in $\mathbb{R P}^{3}$ with respect to rigid isotopies, Funct. Anal. Appl. 18 (1984), no. 1, 49-56.

[Kh8] V. Kharlamov, Nonamphicheiral surfaces of degree 4 in $\mathbb{R P}^{3}$, Lect. Notes Math. 1346 (1988), 349-356.

[Kh9] V. Kharlamov, On a number of components of an $M$-surface of degree 5 in $\mathbb{R P}^{3}$, Proc. of XVI soviet algebraic conference, Leningrad, 1981, pp. 353-354.

[Kh10] V. Kharlamov, Estimates of Betti numbers in topology of real algebraic surfaces, Proc. Internat. Topological Conference, Oberwolfach, 1987, pp. 12-13.

[Kho1] A. G. Khovansky, The index of a polynomial vector field, Funkc. Anal. i Prilozhen. 13 (1979), no. 1, 49-58.

[Kho2] A. G. Khovansky, Boundary indices of polynomial 1-forms with homogeneous components, Algebra i Analiz 10 (1998), no. 3, 193-222.

[Kl] F. Klein, Ueber Flächen dritter Ordnung, Math. Ann. 6 (1873), 551-581.

[KS] K. Kodaira, D. C. Spencer, Groups of complex line bundles over compact Kähler varieties. Divisor class groups on algebraic varieties., Proc. Nat. Acad. Sci. U.S.A. 39 (1953), 868877.

[Ko] A. Korchagin, Classification of real algebraic curves on toric varieties, Preliminary report, 911-th AMS Meeting, Program, Louisiana State Univ., Baton Rouge, Apr. 19-21, 1996, p. 397.

[Kr1] V. A. Krasnov, The Harnack-Thom inequality for mappings of real algebraic varieties, Izv. Akad. Nauk SSSR Ser. Mat. 47 (1983), no. 2, 268-297.

[Kr2] V. A. Krasnov, On the equivariant Grothendieck cohomology of a real algebraic variety and its application, Izv. Ross. Akad. Nauk Ser. Matem. 58 (1994), no. 3, 36-52.

$[\mathrm{Ku}] \quad \mathrm{N}$. Kuiper, The quotient space of $\mathbb{C} P^{2}$ by complex conjugation is the 4-sphere, Math. Ann. 208 (1974), 175-177.

[La] Hon-Fei Lai, Characteristic classes of real manifolds immersed in complex manifolds, Trans. A.M.S. 172 (1972), 1-33.

[LP] F. Laudenbach, V. Poenaru, A note on four-dimensional handlebodies, Bull. Soc. math. France 100 (1972), 337-344.

[Let] M. Letizia, Quotients by complex conjugation of nonsingular quadrics and cubics in $\mathbb{C} P^{3}$ defined over $\mathbb{R}$, Pacif. J. Math. 110 (1984), no. 2, 307-314.

[Ma1] A. Marin, $\mathbb{C} P^{2} / \sigma$ ou Kuiper et Massey au pays des coniques, A la recherche de la topologie perdue (L. Guillou, A. Marin, eds.), Birkhäuser, 1986.

[Ma2] A. Marin, Quelques remarques sur les courbes réelles algébriques planes, A la recherche de la topologie perdue, Russian transl., Mir, 1989, pp. 162-172. (Russian)

[Mas] W. Massey, The quotient space of the complex projective plane under conjugation is a 4-sphere, Geom. Dedicata 2 (1973), 371-374. 
[Mat1] S. Matsuoka, Nonsingular algebraic curves in $\mathbb{R P}^{1} \times \mathbb{R} \mathbb{P}^{1}$, Trans. Amer. Math. Soc. 324 (1991), no. 1, 87-107.

[Mat2] S. Matsuoka, Congruences for $M$ - and $(M-1)$-curves with odd branches on a hyperboloid, Bull. London Math. Soc. 24 (1992), 61-67.

[Mat3] S. Saito (formerly S. Matsuoka), Classification of involutions of lattices with conditions and real algebraic curves on a hyperboloid, preprint, 1-21.

[MS] J. W. Milnor, J. D. Stasheff, Characteristic classes, Annals of Math. Studies, 76, Princeton Univ. Press and Univ. Tokyo Press, Princeton, New Jersey, 1974.

[Mik1] G. Mikhalkin, The complex separation of real surfaces and extensions of Rokhlin congruence, Invent. Math. 118 (1994), 197-222.

[Mik2] G. Mikhalkin, Adjunction inequality for real algebraic curves, Math. Res. Lett. 4 (1997), no. $1,45-52$.

[Mik3] G. Mikhalkin, Congruences for real algebraic curves on an ellipsoid, Adv. Sovient Math., vol. 18, 1994, pp. 223-233.

[Mik4] G. Mikhalkin, Topology of curves of degree 6 on cubic surfaces in $\mathbb{R P}^{3}$, J. Algebraic Geom. 7 (1998), no. 2, 219-237.

$[\mathrm{MH}]$ J. Milnor, D. Husemoller, Symmetric bilinear forms, Springer-Verlag, Berlin-HeidelbergNew York, 1973.

[Na] S. M. Natanzon, Klein surfaces, Uspekhi Mat. Nauk 45 (1990), no. 6, 47-90.

[Nik1] V. V. Nikulin, Integral symmetric bilinear forms and some of their applications, Izv. Akad. Nauk SSSR 43 (1979), no. 1, 117-177 (Russian); English transl. in Math. USSR-Izv. 14 (1980), 103-167.

[Nik2] V. V. Nikulin, Involutions of integral quadratic forms and their application to real algebraic geometry, Izv. Acad. Nauk SSSR 47 (1983), 109-188 (Russian); English transl. in Math. USSR Izv. 22 (1984).

[Och] Ochanine, Signature modulo 16, invariants de Kervaire generalisés et nombres charactéristiques dans la K-théorie reéle, Mem. Soc. Math. France (N.S.) (1981), no. 5.

[Ol] O. A. Oleinik, On the topology of real algebraic curves on an algebraic surface, Matem. Sb. 29 (1951), 133-156. (Russian)

[Or1] S. Orevkov, Link theory and oval arrangements of real algebraic curves, Topology 38 (1999), no. 4, 779-810.

[Or2] S. Orevkov, Riemann existence theorem and construction of real algebraic curves (to appear).

[Pet] I. G. Petrovskiy, On the topology of real plane algebraic curves, Ann. Math 39 (1938), no. 1, 189-209.

[PO] I. G. Petrovskiy, O. A. Oleinik, On the topology of real algebraic surfaces, Isv. Akad. Nauk SSSR, Ser. mat. 13 (1949), 389-402. (Russian)

[Ri] J.-J. Risler, Construction d'hypersurfaces réelles (d'apreès Viro), Astérsique 216 (1993), $69-86$.

[R1] V. A. Rokhlin, Proof of a conjecture of Gudkov, Funkc. Anal. i Prilozhen. 6 (1972), no. 2, $62-64$.

[R2] V. A. Rokhlin, Complex orientation of real algebraic curves, Funkc. Anal. i Prilozhen. 8 (1974), no. 4, 71-75.

[R3] V. A. Rokhlin, Complex topological characteristics of real algebraic curves, Uspekhi Mat. Nauk 33 (1978), no. 5, 77-89.

[R4] V. A. Rokhlin, Congruences modulo 16 in Hilbert's sixteenth problem., Funkc. Anal. i Prilozhen. 6 (1972), no. 4, 58-64.

[R5] V. A. Rokhlin, Congruences modulo 16 in Hilbert's sixteenth problem. II, Funkc. Anal. i Prilozhen. 7 (1973), no. 2, 91-92.

[R6] V. A. Rokhlin, New inequalities in the topology of real plane algebraic curves, Funkc. Anal. i Prilozhen. 14 (1980), no. 1, 37-43.

[S] W. Scharlau, Quadratic and Hermitian forms, Grundlehren der mathematische Wissenschaften, 270, Springer-Verlag, Berlin-New York, 1985.

[Sch] L. Schläfli, On the distribution of surfaces of the third order into species, in reference to the absence or presence of singular points, and the reality of their lines, Phil. Trans. Roy. Soc. London 153 (1863), 195-241.

[Seg] B. Segre, The Non-singular Cubic Surfaces, Oxford University Press, Oxford, 1942. 
[Sil] R. Silhol, Real Algebraic Surfaces, Lecture Notes in Math., vol. 1392, Springer-Verlag, 1989.

[St] J. Steenbrink, Intersection form for quasi-homogeneous singularities, Comp. Math. 34 (1977), no. 2, 211-223.

[SE] N. E. Steenrod, D. B. A. Epstein, Cohomology operations, Princeton University Press, Princeton, 1962.

[Sz] Z. Szabó, Simply-connected irreducible 4-manifolds with no symplectic structure, Invent. Math. 132 (1998), 457-466.

[Th1] R. Thom, Une théorie intrinsèque des puissance de Steenrod, Colloque de Topologie de Strasbourg (1951), exposé N. 6.

[Th2] R. Thom, Espaces fibrés en sphères et carrés de Steenrod, Ann. Éc. Norm. 3 (1952), no. 2, 109-182.

[Th3] R. Thom, Sur l'homologie des variétés algébriques réelles, A symposium in honour of Marston Morse, Princeton Univ. Press, 1965, pp. 255-265.

[Va1] A. N. Varchenko, Theorems on the topological equisingularity of families of algebraic varieties and families of polynomial mappings, Izv. Acad. Nauk SSSR 36 (1972), 957-1019.

[Va2] A. N. Varchenko, Asymptotic Hodge structure on vanishing cohomology, Izv. Akad. nauk SSSR Ser. Mat. 45 (1981), no. 3, 540-591.

[Va3] A. N. Varchenko, Local residue and the intersection form in vanishing cohomology, Izv. Akad. Nauk SSSR, Ser. Matem. 49 (1985), no. 1, 32-54.

[V1] O. Ya. Viro, Real algebraic plane curves: constructions with controlled topology, Leningrad Math. J. 1 (1990), 1059-1134.

[V2] O. Ya. Viro, Construction of M-surfaces, Funct. Anal. Appl. 13 (1979), no. 3.

[V3] O. Ya. Viro, Achievements in the topology of real algebraic varieties in the last six years, Uspekhi Mat. Nauk 41 (1986), no. 3, 45-67.

[V4] O. Ya. Viro, Complex orientations of real algebraic surfaces, Adv. Soviet Math. 18 (1994), 261-284.

[Wl] C. T. C. Wall, Quadratic form in finite groups and related topics, Topology 2 (1964), 281-298.

[Wc] A. Wallace, Linear sections of algebraic varieties, Indiana Univ. Math. J. 20 (1971), $1153-1162$.

[Wa] Sh. Wang, A vanishing theorem for Seiberg-Witten invariants, Math. Res. Lett. 2 (1995), no. $3,305-310$.

[We1] J.-Y. Welschinger, Orientations complexes des J-courbes réelles, Prépublications de l'Univ. Louis Pasteur, Strasbourg (1999).

[We2] J.-Y. Welschinger, J-courbes réelles à nids profonds sur les surfaces réglées, Prépublications de l'Univ. Louis Pasteur, Strasbourg (1999).

[Wi] G. Wilson, Hilbert's sixteenth problem, Topology 17, 53-73.

[Ze] H. G. Zeuthen, Etudes des propriétés de situation des surfaces cubiques, Math. Ann 8 (1875), 1-30.

[Zv1] V. I. Zvonilov, Harlamov's inequalities and Petrovsky-Oleinik inequalities, Funkc. Anal. i Prilozhen. 9 (1975), no. 2, 69-70.

[Zv2] V. I. Zvonilov, Complex topological characteristics of real algebraic curves on surfaces, Funkc. Anal. i Prilozhen. 16 (1982), no. 3, 56-57 (Russian); English transl. in Functional Anal. Appl. 16 (1982), no. 3.

[Zv3] V. I. Zvonilov, Complex topological invariants of real algebraic curves on a hyperboloid and on an ellipsoid, Algebra i Analiz 3 (1991), no. 5 (Russian); English transl. in St. Petersburg Math. J. 3 (1992), no. 5, 1023-1042.

$[\mathrm{Zv} 4] \quad$ V. I. Zvonilov, Stratified spaces of real algebraic curves of bidegree $(m, 1)$ and $(m, 2)$ on a hyperboloid, Amer. Math. Soc. Transl. (2) 173 (1996), 253-264.

Bilkent University, Ankara, Turkey

E-mail address: degt @ fen.bilkent.edu.edu

Institut de Recherche Mathématique Avancée

Université Louis Pasteur et CNRS

7 RUE RENÉ-Descartes

67084, Strasbourg, France

E-mail address: kharlam @ math.u-strasbg.fr 